\title{
Separation of Molybdenum and Copper in Porphyry Deposits The Roles of Sulfur, Redox, and pH in Ore Mineral Deposition at Bingham Canyon
}

\section{Journal Article}

Author(s):

Seo, Jung Hun; Guillong, Marcel (D); Heinrich, Christoph A. (D)

Publication date:

2012-03

Permanent link:

https://doi.org/10.3929/ethz-b-000047367

Rights / license:

In Copyright - Non-Commercial Use Permitted

Originally published in:

Economic Geology 107(2), https://doi.org/10.2113/econgeo.107.2.333 
This is the green Open Access version of: Seo, J. H., Guillong, M. and Heinich, C. A., 2012. Seperation of Molybdenum and Copper in Porphyry Deposits: The Roles of Sulfur, Redox and $\mathrm{pH}$ in Ore Mineral Deposition at Bingham Canyon. Economic Geology, vol. 107, pp. 333356.

https://doi.org/10.2113/econgeo.107.2.333

\title{
Separation of Molybdenum and Copper in Porphyry Deposits: The Roles of Sulfur, Redox and pH in Ore Mineral Deposition at Bingham Canyon
}

\author{
Jung Hun Seo1, Marcel Guillong 1,*, Christoph A. Heinrich ${ }^{1,2}$ \\ 1Department of Earth Sciences, ETH Zurich, 8092 Zurich, Switzerland \\ 2Faculty of Mathematics and Natural Sciences, University of Zurich, Switzerland \\ * Corresponding author: e-mail, seo28@snu.ac.kr \\ Jung Hun SEO (seo@erdw.ethz.ch), Marcel GUILLONG (Marcel.Guillong@utas.edu.au), \\ Christoph A. HEINRICH (heinrich@erdw.ethz.ch)
}

Keywords: Molybdenum, Sulfur, Copper, Fluid inclusion, Porphyry deposit

\begin{abstract}
The giant Bingham Canyon porphyry Cu-Mo-Au deposit (Utah) is associated with Eocene subvolcanic intrusions. It shows a distinct metal zonation above a barren core, with 1) dominantly shallow $\mathrm{Cu}-\mathrm{Au}$ mineralization (Cu-stage) following the early Quartz Monzonite Porphyry (QMP) intrusion and 2) spatially deeper Mo mineralization (Mo-stage) occurring in a separate vein set exclusively after a late Quartz Latite Porphyry (QLP) intrusion that truncates earlier $\mathrm{Cu}-\mathrm{Au}$ veins. To understand this metal separation and the geochemical process of molybdenite mineralization, we investigated fluid inclusions by microthermometry, Raman spectroscopy and laser ablation inductively couple plasma mass spectrometry (LA-ICP-MS) microanalysis in low-grade and high-grade quartz veins of both mineralization stages.

In deep, low-grade quartz veins interpreted to represent the root zone of the $\mathrm{Cu}$-stage we found high concentrations of $\mathrm{Cu}, \mathrm{S}$, and Mo in the fluid inclusions, whereas in low-grade Mostage veins, we found lower $\mathrm{Cu}$, but similar concentrations of $\mathrm{S}$ and $\mathrm{Mo}$, compared to the inferred input fluids to the $\mathrm{Cu}$-stage. Sulfur and copper concentrations were similar in intermediate-density (ID) type fluid inclusions in deep low-grade $\mathrm{Cu}$-stage samples, whereas ID-type inclusions in low-grade Mo-stage veins have S contents that exceed their $\mathrm{Cu}$ contents. In high-grade Mo-stage vein, we found large variations of Mo concentrations in coexisting brine and vapor inclusions. Compared to the P-T conditions of the Cuprecipitation stage (90-260 bars and $320-430{ }^{\circ} \mathrm{C}$ ), the Mo-precipitating fluids were trapped at higher pressures and temperatures of 140-710 bars and 360-580 ${ }^{\circ} \mathrm{C}$. Mass-balance calculation based on the compositions of ID inclusions and brine + vapor assemblages, interpreted to be derived by phase separation during decompression of the ascending
\end{abstract}


single-phase ID fluid, indicate that the mass of vapor phase exceeded that of brine by about 9:1 in both mineralization stages. Combining this mass-balance with the analyzed vapor/brine partitioning data indicates that more than $70 \%$ of Mo, and $S$ (by mass) in the deposit were deposited from the vapor phase. Earlier $\mathrm{Cu}-\mathrm{Au}$ deposition was similarly dominated by vapor, but recently published data about post-entrapment $\mathrm{Cu}$ diffusion in and out of fluid inclusions cast doubt on previous quantifications suggesting that almost none of the copper was deposited by brine.

Mo is less likely to be modified by selective diffusion, and high Mo contents (max. 0.0054 $\mathrm{Mo} / \mathrm{Na}$ in ID; $380 \mu \mathrm{g} / \mathrm{g}$ Mo in brine) in the hydrothermal fluids were maintained from the early $\mathrm{Cu}$-stage to the late Mo-stage. This indicates that Mo concentration was not the decisive factor for separate precipitation of late Mo ore at Bingham Canyon. Instead, the metal separation may be explained by a reduction in redox potential and an increase in acidity in the evolving source region of the fluids, i.e., a large subvolcanic magma reservoir. This is indicated by the stoichiometry of chalcopyrite and molybdenite precipitation reactions, a tentative difference in the $\mathrm{Fe} / \mathrm{Mn}$ ratio in fluids of both veining stages, incipient muscovite alteration along high-temperature molybdenite veins and an increasing tendency for Mo to fractionate from brine to vapor. We suggest that the early $\mathrm{Cu}$-stage fluids were slightly more oxidized and neutral, allowing $\mathrm{Cu}-\mathrm{Fe}$ sulfides to saturate first, while molybdenite saturation was suppressed and Mo was lost from the early ore stage. By contrast during the later Mo-stage, the fluids were more reduced and acidic, thereby allowing selective saturation of molybdenite as the first precipitating sulfide in the cooling and expanding two-phase fluid, consistent with textural observations. This interpretation may imply more generally that small differences in redox potential and acid/base balance of the magmatic source of porphyry-mineralizing systems may be decisive in the temporal and spatial separation of the two metals.

\section{Introduction}

Porphyry-style deposits are the most important $\mathrm{Cu}$ and Mo inventories in the world economically (Singer et al., 2005). Among porphyry-style deposits, most polymetallic $\mathrm{Cu}-$ Mo-Au deposits are associated with compositionally intermediate (e.g. granodiorite, quartz monzonite) subvolcanic intrusions (Beane and Titley, 1981), whereas Mo-only porphyry deposits (Climax-type) are associated with high-silica rhyolite magma intrusions (White et al., 1981). In porphyry deposits that contain Mo as well as $\mathrm{Cu}$, paragenetic relationships of molybdenite and $\mathrm{Cu}$ sulfides from the vein to the orebody scale commonly indicate that molybdenite deposition postdates the main stage of precipitation of $\mathrm{Cu}$-Fe sulfides $\pm \mathrm{Au}$ (Gustafson and Hunt, 1975; Ulrich and Heinrich, 2001; Rusk et al., 2008; Landtwing et al., 2010; Redmond and Einaudi, 2010; Sillitoe, 2010).

Metal ratios including $\mathrm{Cu} / \mathrm{Au}$ and $\mathrm{Mo} / \mathrm{Cu}$ and molybdenite grades are of economic importance, but the geological causes for their variation are not clearly established. The $\mathrm{Cu} / \mathrm{Au}$ ratios in porphyry-style deposits suggested to be influenced by a combination of processes including (1) magma compositions, such as alkaline affinity, redox state, and sulfide melt incorporation (Sillitoe, 1997; Ulrich et al., 1999; Halter et al., 2002; Heinrich et al., 2004; Zajacz et al., 2010), (2) the depth of emplacement affecting the extent of phase separations of magmatic-hydrothermal fluids and metal partitioning (Simon et al., 2006), explaining why most Au-rich porphyries are relatively shallow (Cox and Singer, 1988; Sillitoe, 1997; Landtwing et al., 2010; Murakami et al., 2010), and (3) a mineralogical control on selective extraction of $\mathrm{Au}$ from fluid into $\mathrm{Cu}$-Fe-sulfide solid solutions, notably bornite (Simon et al., 2000; Kesler et al., 2002).

The factors controlling the $\mathrm{Mo} / \mathrm{Cu}$ ratio and the molybdenite grade in porphyry $\mathrm{Cu}-\mathrm{Mo}-\mathrm{Au}$ deposits remain even more elusive. The Mo grades in some deposits show a rough 
correlation with formation depth, i.e., deep porphyry deposits tend to be Au-poor but commonly have high Mo grades, such as Butte, Montana (Singer et al., 2005; Rusk et al., 2008; Murakami et al., 2010). Provinciality and large-scale source processes are indicated by the clustering of the world's largest Mo-rich and Mo-only porphyry deposits in the SWUSA, including Bingham Canyon, Butte, Climax, Henderson, and Questa (Singer et al., 2005). Recent study of $\mathrm{Pb}$ isotopes in rocks and ore-forming fluids suggested that the Momineralizing magmas of this province were produced by melting of ancient subcontinental lithospheric mantle affected by ancient Proterozoic rather than coeval subduction metasomatism (Pettke et al., 2010). The compositional evolution of fluids that formed the Mo-only deposit of Questa showed that their source magmas must have experienced extensive batholith-scale or lower-crustal fractionation (Audétat and Pettke, 2003; Klemm et al., 2008), which may contribute to enrichment of incompatible Mo in residual fluids and explain the typical association Mo-only porphyries with very fractionated magmas (White et al., 1981; Wallace, 1995; Seedorff and Einaudi, 2004a; Audétat, 2010). Additional to a magmatic source control, selective precipitation of molybdenite from the magmatichydrothermal fluids in Mo-rich deposits could be an important factor in metal separation. LA-ICP-MS microanalyses of the fluid inclusions in the Questa deposit showed that the magmatic-hydrothermal fluids contain more $\mathrm{Cu}$ than $\mathrm{Mo}$, although molybdenite was deposited without significant $\mathrm{Cu}$ sulfides (Klemm et al., 2008). High Mo concentrations in brine inclusion assemblages supported the interpretation that $\mathrm{MoS}_{2}$ was dominantly precipitated from brine, whereas $\mathrm{Cu}$ was selectively distilled away from the region of the present Questa orebody by an ascending vapor phase fluid (Klemm et al., 2008).

From hydrothermal experiments, molybdic acid species such as $\mathrm{H}_{2} \mathrm{MoO}_{4}$ were suggested to be important for Mo transport in high-temperature hydrothermal fluids (Candela and Holland, 1984; Keppler and Wyllie, 1991; Rempel et al., 2006; Rempel et al., 2008; Minubayeva and Seward, 2010). While high Mo solubilities up to $1.6 \mathrm{wt} \%$ were reported in sulfur-free saline solutions (Ulrich and Mavrogenes, 2008), much lower Mo solubilities (less than $1 \mathrm{ppm}$ ) were reported in sulfur-bearing multicomponent solubility experiments (Wood et al., 1987; Cao, 1989; Gu, 1993). Sulfur limits hydrothermal Mo mobility through the saturation of molybdenite as a primary magmatic accessory mineral (Bingen and Stein, 2003). Sulfur is an essential element for economically significant precipitation of molybdenite $\left(\mathrm{MoS}_{2}\right)$ from hydrothermal fluids. With the recent analytical advances of quantifying $S$ in single quartz-hosted fluid inclusions by laser ablation inductively coupled plasma mass spectrometry (LA-ICP-MS) (Guillong et al., 2008a; Seo et al., 2011), we showed that magmatic-hydrothermal fluids contain enough sulfur to precipitate sulfide minerals (e.g. $\mathrm{CuFeS}_{2}$ and $\mathrm{MoS}_{2}$ ), but that the concentrations of $\mathrm{S}$ and major chalcophile elements $(\mathrm{Cu}$, Fe) are commonly of similar magnitude (Seo et al., 2009). This opens the possibility that competition between metals and sulfur could be a limiting factor in ore deposition and may contribute to the large-scale separation of metals into distinct ore types (Heinrich, 2006).

The Bingham Canyon porphyry $\mathrm{Cu}-\mathrm{Mo}-\mathrm{Au}$ deposit is particularly suitable for comparing the mechanisms of copper sulfide and molybdenite precipitation because it records temporally well-separated hydrothermal stages of early $\mathrm{Cu}-\mathrm{Au}$ and late Mo mineralization. The molybdenite-bearing quartz veins were formed exclusively after quartz latite porphyry (QLP) dike intrusions, whereas most $\mathrm{Cu}$-Au mineralizing quartz veins predated these dikes (Redmond and Einaudi, 2010). Recent publications on the physical and chemical evolution of the giant Bingham Canyon ore system (Gruen et al., 2010; Landtwing et al., 2010; Redmond and Einaudi, 2010) provide a framework to compare the sulfur, copper, and molybdenum concentrations in fluid inclusion assemblages both from early $\mathrm{Cu}$ - $\mathrm{Au}$-sulfide (Cu-stage) and late molybdenite-bearing (Mo-stage) quartz veins, with the aim of 
understanding the factors that led to the separation of molybdenite from copper sulfides and gold.

\section{Geology and Sampling Strategy}

\section{Geological background of the Bingham Canyon deposit}

The Bingham Canyon $\mathrm{Cu}-\mathrm{Mo}-\mathrm{Au}$ deposit is hosted by series of Late Eocene subvolcanic monzonitic intrusions (collectively called the Bingham Stock) emplaced into Paleozoic sedimentary formations consisting of quartzite and limestone. The Bingham Stock is made up of four main intrusions (Redmond and Einaudi, 2010). The Equigranular Monzonite (EM) is the oldest phase of the Bingham Stock, which did not produce any mineralized veins. The EM was intruded by Quartz Monzonite Porphyry (QMP) followed by the most intense $\mathrm{Cu}-\mathrm{Au}$ mineralization (Cu-stage). After the QMP, intrusion of Latite Porphyry (LP) and Quartz Latite Porphyry (QLP) dikes followed (EM $\rightarrow$ QMP $\rightarrow$ LP $\rightarrow$ QLP). The major Mo-stage mineralization postdated the QLP intrusion (Gruen et al., 2010; Landtwing et al., 2010; Redmond and Einaudi, 2010).

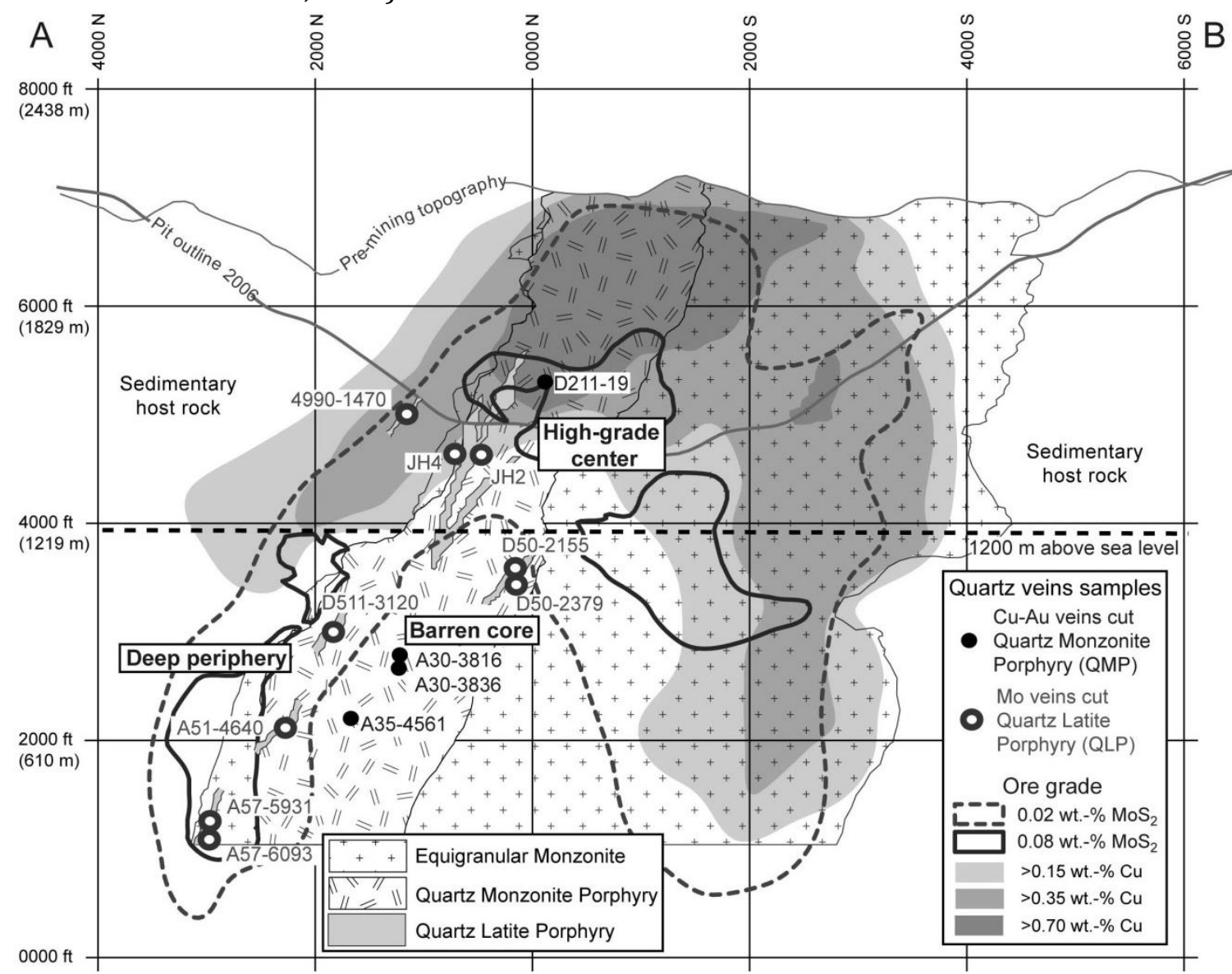

Figure 1. Northwest-southeast cross section through Bingham Canyon (refer to A-B in Fig.7 of Gruen et al., 2010 for section location) showing lithologies of the Bingham Stock, $\mathrm{Cu}$ and $\mathrm{MoS}_{2}$ ore grade contours, and locations of quartz vein samples with respect to the sampling regions discussed in the text. The Cu- stage quartz stockwork veins were sampled from quartz monzonite porphyry (QMP) and late-stage quartz latite porphyry (QLP) dikes (sample 4990-1470), whereas all Mo-stage quartz-molybdenite veins were sampled from quartz latite porphyry. Samples A30-3816, 3836 (Cu-stage) and D50-2155, 2379 (Mo-stage) are from the barren core (elevation below 1,200 m). Samples D211-19 (Cu-stage), JH2, JH4 (Mo-stage), and 4990-1470 (post-QLP Cu-stage and Mo-stage) are from the high-grade center (elevation greater than 1,200 m). Samples A57-5931, 6093, A51-4640, and D5113120 (Mo-stage) are from the deep periphery of the deposit (elevation below 1,200 m). 
The $\mathrm{Cu}$-rich (contour of $0.15 \mathrm{wt} \%$ of $\mathrm{Cu}$ ) ore body at Bingham Canyon deposit is bell-shaped. The central highest-grade $\mathrm{Cu}$ ore body $(>0.70 \mathrm{wt} \%$ of $\mathrm{Cu}$ ) additionally contains high $\mathrm{Au}$ grades (contours of 0.30 and 1.00 ppm of Au; Fig. 1; Gruen et al., 2010; Landtwing et al., 2010). Molybdenite is concentrated deeper and inward compared to $\mathrm{Cu}-\mathrm{Au}$ mineralization, but the shape of the Mo-rich ore zone (contour of $0.08 \mathrm{wt} \% \mathrm{MoS}_{2}$ ) resembles that of the Curich shell (Fig. 1). While the highest-grade $\mathrm{Cu}$ accumulated around the central part of the mine, high grade Mo (contour of $0.02 \mathrm{wt} \% \mathrm{MoS}_{2}$ ) zones are more broadly distributed from the central to the peripheral part. At the top of the deposit, the center of the high grade Morich zone partially overlaps with that of the high-grade Cu zone $(0.70 \mathrm{wt} \% \mathrm{Cu})$ (Fig. 1$)$. Copper-gold mineralization without any discernable molybdenite is associated with quartz stockwork veining and pervasive potassic alteration. Most $\mathrm{Cu}$-Au stage stockwork veins are contemporaneous with or postdate the intrusion of Quartz Monzonite Porphyry (Fig. 2) but stockwork veining with diminishing intensity followed the intrusion of Latite Porphyry and Quartz Latite Porphyry. Molybdenite mineralization of a distinct Mo-stage formed typically 10-60 mm thick quartz-molybdenite veins. Theses veins consistently postdate the late intrusion of Quartz Latite Porphyry wherever we checked areas of economic Mo grades that also contain the Quartz Latite Porphyry dikes. These quartz-molybdenite veins typically contain euhedral and partly free-standing quartz crystals (3-2 in Fig. 2). In the vuggy center of the quartz-molybdenite veins, minor late chalcopyrite is precipitated as euhedral crystals (3-2 in Fig. 2B and Fig. 2C), locally overgrown by calcite. Most quartz-molybdenite veins occur in areas of pervasive biotite alteration, which may at least in part be related to earlier $\mathrm{Cu}-\mathrm{Au}$ stage stockwork veining and its barren core. At least locally, previously weakly altered wall rocks next to quartz-molybdenite veins show a bleached halo containing finegrained muscovite (Fig. 2C). All generations of quartz $\mathrm{Cu}-\mathrm{Au}$ stockwork veins and the late quartz-molybdenite veins have been cut by quartz-pyrite ( \pm chalcopyrite \pm calcite) veins with broad haloes of feldspar-destructive alteration (sericite \pm clay minerals; QSP veins) (Redmond and Einaudi, 2010).

\section{Sample descriptions}

We sampled quartz veins of three paragenetic stages that show clear and consistent time sequences of emplacement in drill core and in outcrop: (1) Quartz stockwork veins associated with the main $\mathrm{Cu}-\mathrm{Au}$ mineralization, hosted by QMP and early EM, (2) late-stage quartz stockwork veins that clearly postdate the latest QLP dikes and are associated with minor late copper mineralization, and (3) quartz-molybdenite veins that consistently postdate QLP at each sampling site and also cut the late stockwork veins where present. We sampled these vein generations in the low grade center of the deposit (barren core), in deep Mo-rich peripheral zone accessible in drill core only (deep periphery), and in the high-grade $\mathrm{Cu}-\mathrm{Au}$ and Mo rich center of the open pit (high-grade center; Fig. 1). From a greater number of reconnaissance samples, 13 veins were studied in detail, to obtain textural information and quantitative data from 115 fluid inclusion assemblages comprising 3 to 10 individual inclusions each (Table 1, 2, 3, 4).

Deep quartz stockwork veins (Cu-stage in barren core; A30-3816, 3836, and A35-4561) are located lower than 1,200 $\mathrm{m}$ above sea level in the center of the mine. They contain minor amounts of disseminated pyrite and chalcopyrite in potassically altered QMP. Based on mineralogy and similar textural appearance (variably irregular, sugary-texture without vugs), these deep quartz stockwork veins were interpreted to be the channelways of fluids generating the main $\mathrm{Cu}-\mathrm{Au}$ stage mineralization in the higher part of the system (Landtwing et al., 2010). These veins usually contain three types of fluid inclusions: predominant $\mathrm{CO}_{2}$ bearing intermediate-density fluid inclusions, and less abundant vapor and brine inclusions (Redmond et al., 2004). A high-grade Cu-Au quartz stockwork vein (D211-19) hosted by K- 

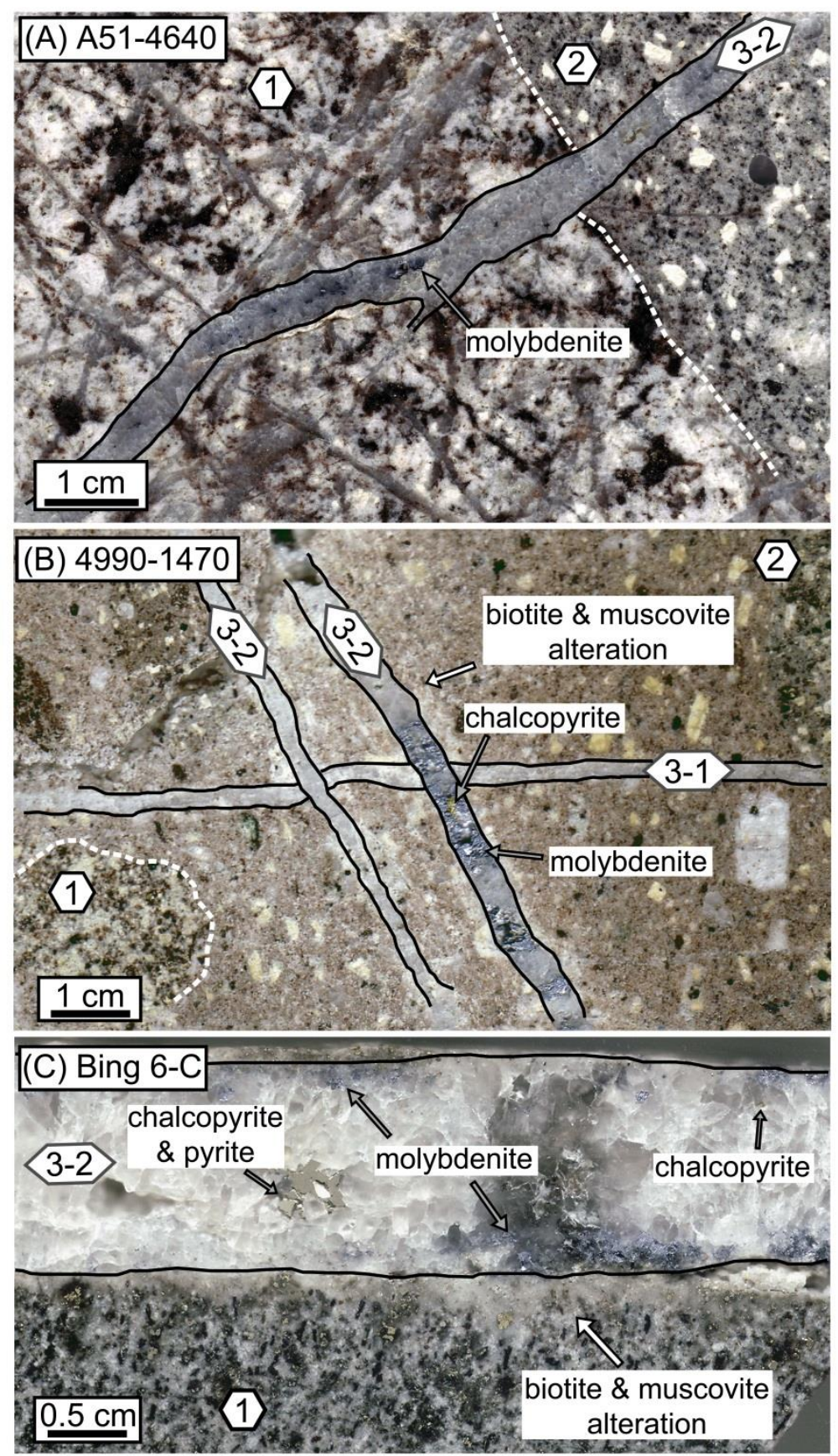

Figure 2. Photograph of polished slabs showing temporal relationships between intrusions, quartz veins, and alteration assemblages. (A) Quartz Monzonite Porphyry (1) has been cut first by quartz stockwork veinlets, then intruded by Quartz Latite Porphyry (2) and finally cut by a quartz-molybdenite vein (3-2) (deep periphery, A514640), (B) Quartz Latite Porphyry (2) with quartz stockwork veined Quartz Monzonite Porphyry clasts (1) is cut by a late quartz stockwork vein (3-1) and quartz molybdenite veins (3-2) (shallow peripheral/central zone, 4990-1470). (C) Quartz-molybdenite vein is hosted in Quartz Monzonite Porphyry (1) (high-grade center, Bing 6-C).

The Quartz Monzonite Porphyry intrusion with pervasive potassic alteration introduced by abundant quartz stockwork veining (1) which predates the Quartz Latite Porphyry dike (2), and quartz-molybdenite vein crosscut the previous intrusions and veins (3-2). Cu-bearing late-stage quartz stockwork vein (3-1) hosted in late Quartz Latite Porphyry dike (2) and the vein is cut by vuggy quartz-molybdenite vein (3-2). Late euhedral chalcopyrite occur at the centerline of some quartz-molybdenite veins (e. g. 3-2 in B and C). Biotite and muscovite alteration halos occur quartz-molybdenite veins. White dashed line represent contact of Quartz Monzonite Porphyry - Quartz Latite Porphyry and black solid line represent quartz veins. 
feldspar and biotite altered QMP comes from the center of the deposit at 1,600 m above sea level. Its blocky quartz grains contain chalcopyrite and bornite precipitated at an interstitial paragenetic position between an early re-dissolved and a later re-cementing generation of vein quartz (Landtwing et al., 2005; Landtwing et al., 2010). The high-grade quartz vein contains predominantly vapor and brine inclusions that are partly trapped on single healed microfractures as cogenetic vapor and brine (boiling assemblage).

Although most $\mathrm{Cu}$-bearing quartz stockwork veins formed before the QLP intrusion, latestage quartz stockwork veins of otherwise similar appearance cut the QLP intrusion and contain a minor amount of $\mathrm{Cu}$ minerals (post-QLP Cu-stage; 3-1 in Fig. 2B), including bornite and digenite. These late-stage quartz stockwork veins are usually less than $10 \mathrm{~mm}$ wide and have been crosscut by high-grade quartz molybdenite veins (3-2 in Fig. 2B). Fluid inclusion types in the late-stage QLP-hosted quartz stockwork vein are mostly vapor and brine inclusions, locally as cogenetic vapor+brine pairs.

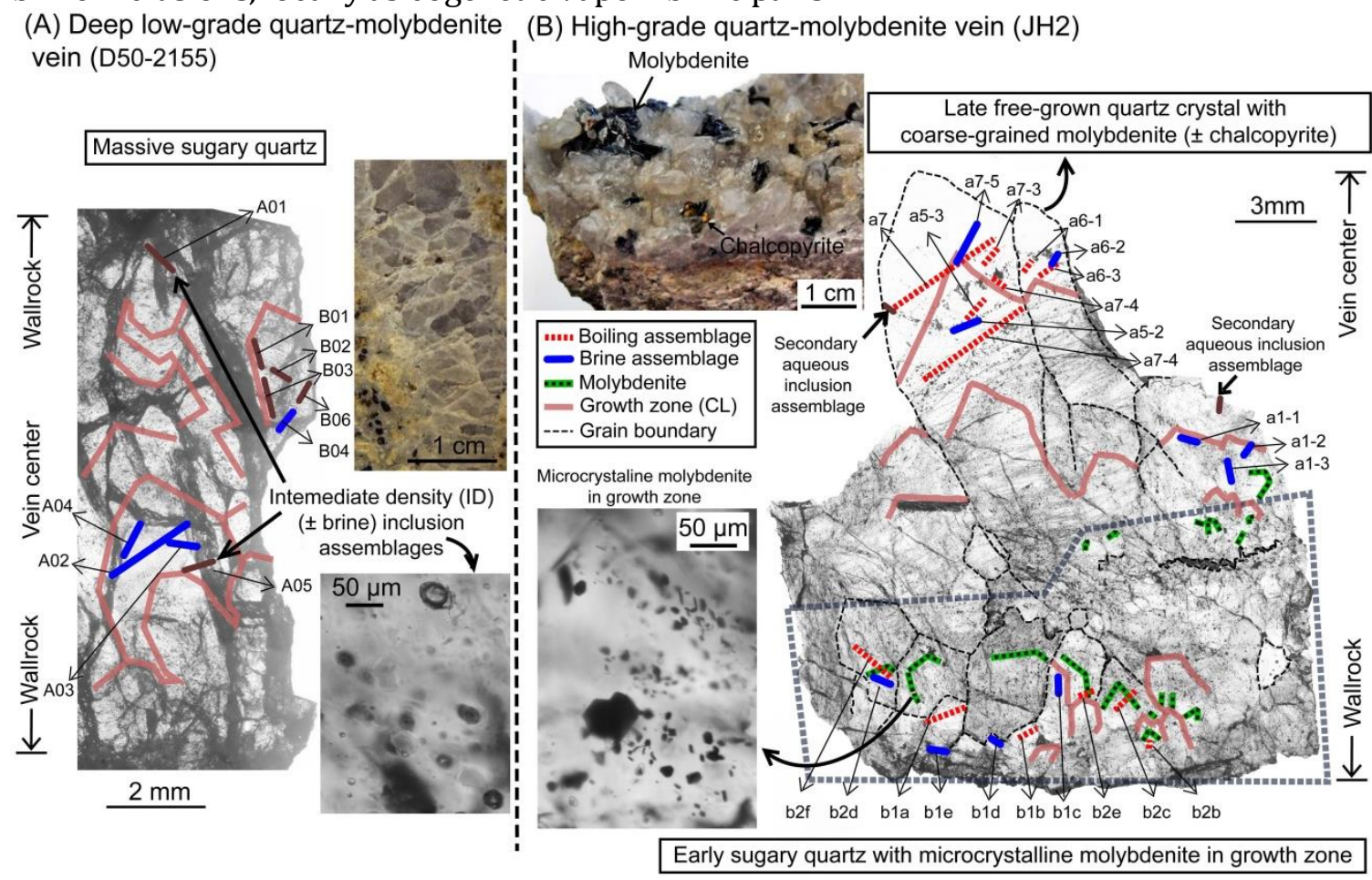

Figure 3. Quartz vein textures and locations of fluid inclusion assemblages in the low-grade (A; D50-2155) and high-grade (B; JH2) quartz-molybdenite veins. Growth zones in quartz were determined from a cathodoluminescence image taken by SEM, and by optical microscopy. (A) Massive sugary quartz crystal grown in the deep low-grade quartz-molybdenite vein contains intermediate-density fluid inclusions. Some intermediatedensity fluid inclusions are associated with brine inclusions. (B) Two stages of quartz textures were observed in the high grade quartz-molybdenite vein. Early sugary quartz crystals grown along the wallrock have microcrystalline molybdenite in growth-zone, which is texturally similar to the low grade quartz-molybdenite vein (A). The early massive sugary quartz crystals were overgrown by texturally-late euhedral free-standing quartz crystals. This free-standing quartz crystals are closely associated with precipitations of coarse-grained molybdenite and local precipitation of euhedral chalcopyrite in the vein center, see the sample figure at (B). Numerous pseudo-secondary or secondary brines, vapors, and boiling assemblages are associated with early sugary quartz crystals and late free-standing quartz crystals.

Low and high-grade quartz-molybdenite veins were sampled where we had clear evidence that they cut late QLP dikes and their contacts with earlier lithologies (EM, QMP, and LP). Low-grade quartz-molybdenite veins (Low-grade Mo-stage; D50-2155, 2379) were sampled in the deep barren core of the mine (elevations of 1,000-1,100 $\mathrm{m}$ above sea level). They were selected because they showed the same crosscutting relationship and contain a minor 
amount of molybdenite, as tiny dispersed crystals (D50-2155) or as a fine selvage of molybdenite grown from the wallrock (D50-2379). These weakly Mo-mineralized veins are interpreted to be low-grade versions of the main-stage (high-grade) quartz-molybdenite veins located higher up in the deposit. They contain $\mathrm{CO}_{2}$-bearing intermediate-density inclusions (Fig. 3A) and some brine inclusions. High-grade quartz-molybdenite veins (Highgrade Mo-stage; JH2, JH4, D511-3120, A51-4640, 4990-1470, A57-5931, A57-6093 and D511-3120) were collected from the open pit surface at 2007 and from drill cores of the deep periphery in the mine (Fig. 1). High-grade quartz-molybdenite veins sampled from the high-grade center (elevations of 1,400-1,500 m above sea level; JH2, JH4, and 4990-1470) and contain mainly brine and vapor inclusions, whereas samples from the deep periphery (elevations of 300-800 m above sea level; A51-4640, A57-5931, A57-6093 and D511-3120) contain intermediate-density fluid inclusions.

In high-grade quartz-molybdenite veins, molybdenite precipitated throughout the paragenetic sequence. Early microcrystalline or massive flaky molybdenite commonly overgrew the contact with the wallrock and predated sugary quartz (Fig. 3B). Fine-grained sugary quartz was overgrown by euhedral quartz crystals. Coarse flakes of molybdenite were deposited at the base of the large euhedral quartz crystals overgrowing sugary quartz (Fig. 3B) or as hexagonal platelets on top of the quartz crystals in the vein center (Fig. 2A). In the central vug of many quartz-molybdenite veins, euhedral chalcopyrite and finally calcite have overgrown quartz and molybdenite (Fig. 2C). Quartz veins of all generations contain vapor, brine, and commonly coexisting vapor and brine inclusions. The quartz molybdenite veins are hosted in potassic alteration of QLP, but commonly show a weak halo of overprinting muscovite or clay alteration (Table 1).

Textures were studied by optical microscopy and cathodoluminescence images taken by scanning electron microscopy (Fig. 3, 4). Low-grade quartz-molybdenite veins located at the deep barren core have massive quartz without open vugs (Fig. 4A). A cathodoluminescence image of this sugary quartz reveals microscopic euhedral growth perpendicularly away from the wall rock (Fig. 4A). Similar texture can be observed in the basal zone of a highgrade quartz-molybdenite vein (sugary quartz; Fig. 3B, 4B, 4C), intergrown with microcrystalline molybdenite along growth zones of quartz. The sugary quartz zone is overgrown by texturally similar, but coarser-grained euhedral free-standing quartz crystals and large flakes of molybdenite (Fig. 3B and Fig. 4B). These textures confirm that the two quartz generations (sugary and free-standing quartz) overlapped with molybdenite deposition temporally. Cathodoluminescence images of some of the quartz-molybdenite veins show evidence of minor redissolution and reprecipitation of a second generation of quartz (Fig. 4), similar to the Cu-stage quartz stockwork veins (Redmond et al., 2004; Landtwing et al., 2005; Landtwing et al., 2010).

\section{Methods}

Representative fluid inclusion assemblages (FIA) were selected by detailed petrography and checked by microthermometry to confirm that they show consistent microthermetric behavior, such as halite dissolution and homogenization temperatures. Obtaining microthermometric properties of $\mathrm{CO}_{2}$-bearing vapor inclusions was, in most cases, difficult because of thin film of aqueous liquid lining the walls of the inclusions and the formation of clathrates. Homogenization temperatures of vapor inclusions were assumed to be equivalent to those of coexisting brine inclusions in sets of texturally-proven cogenetic brine and vapor "boiling" assemblage. Homogenization temperatures of the brine inclusions in the boiling assemblages were combined with the apparent brine salinities ( $w \mathrm{t} \% \mathrm{NaCl}$ eq.) to estimate the fluid pressure of the entrapment condition, based on the two-phase surface $\mathrm{NaCl}-\mathrm{H}_{2} \mathrm{O}$ model system (Driesner and Heinrich, 2007). 
Table 1. Descriptions of the quartz vein samples from drill core and mine outcrops that were used for fluid inclusion analyses.

\begin{tabular}{|c|c|c|c|}
\hline sample \# & host rock & $\begin{array}{l}\text { location, elevation } \\
\text { above sea level }\end{array}$ & mineralogy, quartz texture, alteration \\
\hline \multicolumn{4}{|c|}{ Deep, quartz stockwork vein at barren core (low-grade Cu-stage) } \\
\hline A35-4561 & QMP & Central, $650 \mathrm{~m}$ & $8 \mathrm{~mm}$ blocky fine grain quartz vein, chalcopyrite \pm pyrite. Biotite alteration \\
\hline A30-3816 & QMP & Central, $750 \mathrm{~m}$ & $1 \mathrm{~cm}$ blocky quartz vein with chalcopyrite \pm pyrite. Biotite alteration \\
\hline A30-3836 & QMP & Central, $750 \mathrm{~m}$ & $1 \mathrm{~cm}$ blocky quartz vein with chalcopyrite \pm pyrite. Biotite alteration \\
\hline \multicolumn{4}{|c|}{ Quartz stockwork vein at high-grade center (high-grade Cu-stage) } \\
\hline D211-19 & QMP & Central, $1600 \mathrm{~m}$ & $\begin{array}{l}\text { Gray-clear blocky (sometimes vuggy) quartz vein with chalcopy rite (chalcopyrite occur at } \\
\text { the interstitials of early generation of quartz; Landtwing et al., 2005). Biotite alteration. }\end{array}$ \\
\hline \multicolumn{4}{|c|}{ Deep, quartz-molybdenite vein at barren core (low-grade Mo-stage) } \\
\hline D50-2155 & QLP & Central, $1090 \mathrm{~m}$ & $1 \mathrm{~cm}$ fine to coarse grain blocky quartz vein \pm molybdenite. Weak muscovite alteration. \\
\hline D50-2379 & QLP & Central, $1024 \mathrm{~m}$ & $\begin{array}{l}1 \mathrm{~cm} \text { coarse grain blocky quartz vein with molybdenite } \pm \text { pyrite (molybdenite grown from } \\
\text { the wallrock). Biotite \& muscovite alteration. }\end{array}$ \\
\hline \multicolumn{4}{|c|}{ Quartz-molybdenite vein at high-grade center and deep periphery (high-grade Mo-stage) } \\
\hline $\begin{array}{l}4990-1470(2- \\
\text { types of veins) }\end{array}$ & QLP & Central, $1500 \mathrm{~m}$ & $\begin{array}{l}\text { QMP clast entrained in QLP host. Two generations of quartz veins. 1) Post-QLP late } \\
\text { stockwork vein with gray-clear quartz (digenite) vein with bo rnite alterations crosscut by } 2 \text { ) } \\
\text { buggy and clear quartz-molybdenite vein (molybdenite grown from the wallrock and late } \\
\text { euhedral chalcopyrite occured at vein center). Weak muscovite alteration around the quartz- } \\
\text { molybdenite vein. }\end{array}$ \\
\hline $\mathrm{JH} 2$ & QLP & Central, $1430 \mathrm{~m}$ & $\begin{array}{l}\text { Two generatons of molybdenite deposition at the single vein; 1) microcrystaline } \\
\text { molybdenite within growthzone of early sugary (blocky) quartz overgrown by } 2 \text { ) euhedral, } \\
\text { freestanding quartz vein with coarser molybdenite } \pm \text { late euhedral chalcopyrite. The vein } \\
\text { cuts early stockwork veinlets. Muscovite alteration around quartz-molybdenite vein. }\end{array}$ \\
\hline
\end{tabular}




\begin{tabular}{ccll}
\hline JH4 & EM\&QLP & Central, 1460 m & $\begin{array}{l}\text { Thick quartz vein with freestanding quartz crystals with flakes of molybdenite interstitially } \\
\text { growned } \pm \text { late euhedral chalcopyrite. The vein cuts also EM. Muscovite alteration. }\end{array}$ \\
\hline D511-3120 & QLP & Peripheral, $830 \mathrm{~m}$ & $\begin{array}{l}\text { 8 mm coarse grain blocky quartz vein with molybdenite (molybdenite grown at centerline). } \\
\text { Vug filled calcite. Muscovite alteration. The vein cuts early biotite-altered quartz veinlets. }\end{array}$ \\
\hline A51-4640 & QMP\&QLP & Peripheral, 640 m & $\begin{array}{l}\text { Contact of QLP and QMP. 8 mm of clear (buggy sometimes) quartz -molybdenite veinlet } \\
\text { (molybdenite grown at centerline) cutting QMP hosted stockwo rk veinlets and QLP. Weak } \\
\text { muscovite alteration. }\end{array}$ \\
\hline A57-5931 & QMP\&QLP & Peripheral, 330 m & $\begin{array}{l}\text { 8 mm fine sugary quartz vein with molybdenite (molybdenite grown at centerline). The vein } \\
\text { cuts contact of QMP and QLP. Biotite alteration }\end{array}$ \\
\hline A57-6093 & QMP\&QLP & Peripheral, 350 m & $\begin{array}{l}\text { Two generations of quartz veins; 1) 6 mm milky quartz vein, 2) 1 mm two fine -grained } \\
\text { quartz moybdenite veins } \pm \text { pyrite. These cut the earlier milky quartz vein. Biotite alteration. }\end{array}$ \\
\hline \hline
\end{tabular}

$\mathrm{EM}=$ equigranular monzonite, $\mathrm{QMP}=$ quartz monzonite porphyry, $\mathrm{QLP}=$ quartz latite porphyry. 
Table 2. Composition of $\mathrm{Cu}$-stage (quartz stockwork veins) brine inclusion assemblages in the Bingham Canyon deposit.

\begin{tabular}{|c|c|c|c|c|c|c|c|c|c|c|c|c|c|c|c|c|c|}
\hline$\mu \mathrm{g} / \mathrm{g}$ & Location & Sample\# & Sal. & $\operatorname{Th}\left({ }^{\circ} \mathrm{C}\right)$ & $\mathbf{P}($ bar) & ${ }^{23} \mathrm{Na}$ & ${ }^{32} \mathbf{S}$ & ${ }^{39} \mathrm{~K}$ & ${ }^{57} \mathrm{Fe}$ & ${ }^{65} \mathrm{Cu}$ & ${ }^{75} \mathrm{As}$ & ${ }^{133} \mathrm{Cs}$ & ${ }^{197} \mathrm{Au}$ & ${ }^{208} \mathrm{~Pb}$ & ${ }^{66} \mathrm{Zn}$ & ${ }^{95} \mathrm{Mo}$ & mark \\
\hline Av. & center & A30-3816 & 41.9 & 332 & ND & 120000 & 8400 & 96000 & 50000 & 10000 & 46 & 49 & 0.26 & 3300 & 3400 & 6.6 & boil \\
\hline SD & Deep stw. & 4_TP7 & 0.6 & 13 & & 3100 & 4700 & 7800 & 4300 & 1000 & 7 & 9 & 0.12 & 290 & 370 & 4.0 & \\
\hline Av. & center & A30-3816 & 40.4 & 389 & 173 & 120000 & 4800 & 100000 & 39000 & 7800 & 30 & 48 & 0.42 & 3300 & 2700 & 170 & boil \\
\hline SD & Deep stw. & 4_TP8 & 0.8 & 6 & realPT & 2100 & 1600 & 5400 & 3100 & 2500 & 5 & 6 & 0.13 & 160 & 340 & 100 & \\
\hline Av. & center & A30-3816 & 39.0 & 380 & 162 & 110000 & 6400 & 100000 & 50000 & 6000 & 40 & 49 & 0.24 & 2900 & 3800 & 12 & boil \\
\hline SD & Deep stw. & 4_TP9(b) & 0.6 & 25 & realPT & 1300 & 2200 & 3400 & 13000 & 6400 & 15 & 12 & 0.12 & 140 & 1400 & 15 & \\
\hline Av. & center & A30-3816 & 36.5 & 374 & 155 & 100000 & 6100 & 100000 & 40000 & 7700 & 37 & 42 & 0.21 & 3500 & 3300 & 51 & boil \\
\hline SD & Deep stw. & 4_TP10(b) & 0.6 & 16 & realPT & 6200 & 2900 & 15000 & 8800 & 2900 & 12 & 19 & 0.23 & 590 & 1600 & 71 & \\
\hline Av. & center & A30-3816 & 36.1 & 381 & 168 & 97000 & 12000 & 110000 & 50000 & 10000 & 38 & 53 & 0.22 & 3100 & 4100 & 50 & boil \\
\hline SD & Deep stw. & 4_TP11(b) & 0.4 & 15 & realPT & 1300 & 4200 & 4100 & 4400 & 1400 & 3 & 6 & 0.09 & 330 & 550 & 31 & \\
\hline Av. & center & A35-4561 & 34.4 & 323 & 88 & 97000 & 7600 & 52000 & 36000 & 7600 & 57 & 64 & 0.14 & 2800 & ND & 440 & boil \\
\hline SD & Deep stw. & 1-TP2(b) & 1.8 & 15 & realPT & 17000 & 4500 & 42000 & 11000 & 6100 & 37 & 47 & 0.06 & 1700 & ND & 470 & \\
\hline Av. & center & A35-4561 & 36.8 & 379 & 162 & 120000 & 5200 & 61000 & 31000 & 5700 & 33 & 39 & 0.43 & 2300 & 2300 & 380 & boil \\
\hline SD & Deep stw. & 1_TP3 & 1.4 & 13 & realPT & 2100 & 1700 & 5600 & 2100 & 210 & 7 & 6 & 0.09 & 76 & 180 & 24 & \\
\hline Av. & center & A35-4561 & 30.0 & 379 & 176 & 100000 & 8200 & 47000 & 36000 & 7600 & 59 & 33 & ND & 2100 & 3000 & 59 & boil \\
\hline SD & Deep stw. & 1_TP4 & 0.6 & 14 & realPT & 2500 & 4400 & 6100 & 3800 & 1200 & 34 & 17 & ND & 100 & 760 & 19 & \\
\hline Av. & center & A35-4561 & 34.3 & 363 & 142 & 110000 & 7400 & 57000 & 33000 & 7900 & 25 & 23 & ND & 2400 & 2800 & 330 & boil \\
\hline SD & Deep stw. & 1_TP5 & 1.5 & 26 & realPT & 5800 & 280 & 14000 & 9100 & 4000 & ND & 17 & ND & 450 & 1000 & 20 & \\
\hline Av. & center & A35-4561 & 36.0 & 363 & 138 & 110000 & 3600 & 65000 & 45000 & 3900 & 38 & 45 & 1.10 & 2700 & 2800 & 220 & boil \\
\hline SD & Deep stw. & 1_TP6 & 0.9 & 8 & realPT & 990 & 1800 & 2300 & 17000 & 1500 & 6 & 4 & 0.80 & 340 & 670 & 160 & \\
\hline Av. & center & A30-3836 & 39.2 & 313 & ND & 110000 & 3700 & 100000 & 49000 & 7500 & 49 & 52 & 0.80 & 3100 & 2400 & 330 & no-boil \\
\hline SD & Deep stw. & 1_B1 & 0.0 & 15 & & 7700 & 1300 & 19000 & 11000 & 1400 & 10 & 13 & ND & 550 & 660 & 160 & \\
\hline Av. & center & A30-3836 & 38.7 & 371 & 146 & 110000 & 4300 & 100000 & 49000 & 7400 & 37 & 49 & 0.49 & 3200 & 2700 & 340 & no-boil \\
\hline SD & Deep stw. & 1_B2 & 0.4 & 18 & $\min P T$ & 3400 & 1600 & 8600 & 3000 & 3800 & 5 & 6 & 0.27 & 530 & 750 & 95 & \\
\hline Av. & center & A30-3836 & 37.9 & 328 & 89 & 110000 & 10000 & 82000 & 39000 & 9500 & 13 & 43 & 1.80 & 2800 & 2100 & 180 & no-boil \\
\hline SD & Deep stw. & 1_B3 & 0.9 & 3 & $\min P T$ & 4500 & 7700 & 11000 & 2700 & 2000 & ND & 8 & ND & 63 & 250 & 110 & \\
\hline Av. & center & A30-3836 & 40.1 & 326 & ND & 120000 & 3600 & 98000 & 41000 & 7200 & 33 & 44 & 0.69 & 2900 & 3500 & 120 & no-boil \\
\hline SD & Deep stw. & 1_TP1 & 0.2 & 14 & & 9000 & 2200 & 21000 & 7800 & 2800 & 16 & 18 & ND & 880 & 1900 & 20 & \\
\hline Av. & center & D211-19 & 50.9 & 369 & ND & 150000 & 2500 & 120000 & 56000 & 450 & 110 & 53 & 2.00 & 5000 & 4500 & 6.6 & no-boil \\
\hline SD & Cu-vein & 1_B1 & 2.8 & 26 & & 4500 & 550 & 11000 & 12000 & 170 & 37 & 7 & ND & 840 & 940 & 7.6 & \\
\hline
\end{tabular}




\begin{tabular}{|c|c|c|c|c|c|c|c|c|c|c|c|c|c|c|c|c|c|}
\hline Av. & center & D211-19 & 47.8 & 385 & ND & 130000 & 11000 & 120000 & 72000 & 1100 & 35 & 42 & ND & 4800 & 4300 & 42.0 & boil \\
\hline SD & Cu-vein & 1_B2 & 0.8 & 14 & & 7500 & 3000 & 18000 & 4600 & 280 & 22 & 6 & ND & 1100 & 1000 & 5.7 & \\
\hline Av. & center & D211-19 & 52.9 & 400 & ND & 140000 & 5600 & 150000 & 94000 & 2800 & 44 & 60 & 0.31 & 5900 & 5900 & 47.0 & boil \\
\hline SD & Cu-vein & 1_B3 & 2.0 & 16 & & 13000 & 3000 & 30000 & 29000 & 2600 & 19 & 17 & 0.17 & 2300 & 3100 & 38.0 & \\
\hline Av. & center & D211-19 & 52.2 & 392 & ND & 150000 & 5000 & 120000 & 96000 & 2800 & 38 & 52 & 1.00 & 5700 & 5500 & 100.0 & boil \\
\hline SD & Cu-vein & 1_B4 & 1.3 & 8 & & 2600 & 1900 & 3400 & 11000 & 670 & 13 & 11 & 0.64 & 1300 & 1500 & 110.0 & \\
\hline Av. & center & D211-19 & 51.8 & 373 & ND & 140000 & 2600 & 140000 & 140000 & 870 & 61 & 110 & 0.34 & 6700 & 7000 & 6.7 & boil \\
\hline SD & Cu-vein & 1_B5 & 0.1 & 23 & & 9200 & 1400 & 22000 & 40000 & 300 & 14 & 39 & ND & 1800 & 1800 & 7.9 & \\
\hline Av. & center & D211-19 & 49.6 & 378 & ND & 140000 & 7100 & 130000 & 62000 & 1300 & 70 & 42 & ND & 4100 & 3800 & 19.0 & boil \\
\hline SD & Cu-vein & 1_B6 & ND & ND & & 8700 & 3500 & 22000 & 25000 & 760 & 45 & 18 & ND & 1000 & 980 & 17.0 & \\
\hline Av. & center & D211-19 & 43.3 & 393 & 174 & 120000 & 5400 & 110000 & 61000 & 5700 & 54 & 36 & ND & 4100 & 3600 & 29.0 & no-boil \\
\hline SD & Cu-vein & 1_B7 & 4.6 & 22 & $\min P T$ & 4800 & 3500 & 10000 & 33000 & 3700 & 40 & 1 & ND & 1500 & 1200 & ND & \\
\hline Av. & center & D211-19 & 43.3 & 393 & 174 & 110000 & 15000 & 130000 & 100000 & 18000 & 71 & 40 & ND & 5900 & 5500 & 160.0 & no-boil \\
\hline SD & Cu-vein & 1_B7-1 & 4.6 & 22 & $\min P T$ & 9400 & 5000 & 25000 & 5200 & 12000 & 26 & 9 & ND & 860 & 540 & 180.0 & \\
\hline Av. & center & D211-19 & 39.7 & 430 & 261 & 110000 & 7200 & 98000 & 65000 & 1000 & 35 & 34 & ND & 3500 & 3000 & 56.0 & boil \\
\hline SD & Cu-vein & 1_B8 & 1.1 & 7 & realPT & 1900 & 430 & 4700 & 2000 & 200 & 7 & 8 & ND & 360 & 180 & 66.0 & \\
\hline Av. & center & D211-19 & 43.1 & 417 & 221 & 120000 & 5500 & 110000 & 64000 & 920 & 47 & 47 & 0.30 & 3800 & 2700 & 100.0 & no-boil \\
\hline SD & Cu-vein & 1_B9 & 0.2 & 9 & $\min P T$ & 3800 & 1300 & 8600 & 6800 & 150 & 15 & 2 & 0.26 & 880 & 1100 & 43.0 & \\
\hline Av. & center & D211-19 & 46.2 & 410 & 196 & 140000 & 4900 & 100000 & 45000 & 2500 & 71 & 47 & 0.10 & 3300 & 2900 & 360.0 & boil \\
\hline SD & Cu-vein & 1_B10 & 0.8 & 28 & realPT & $\mathrm{ND}$ & ND & ND & ND & ND & $\mathrm{ND}$ & ND & ND & $\mathrm{ND}$ & $\mathrm{ND}$ & $\mathrm{ND}$ & \\
\hline Av. & center & $4990-1470$ & 43.7 & 391 & 170 & 120000 & 2900 & 110000 & 47000 & 560 & 56 & 39 & 0.76 & 3500 & 3000 & 83 & no-boil \\
\hline SD & QLP stw. & 1_B1 & 0.8 & 3 & $\min \mathrm{PT}$ & 1000 & 1500 & 2000 & 1200 & 14 & 1.4 & 3 & 0.65 & 220 & 460 & 2 & \\
\hline Av. & center & $4990-1470$ & 39.7 & 364 & 134 & 110000 & 4800 & 93000 & 51000 & 1000 & 34 & 46 & 0.23 & 2700 & 2300 & 72 & no-boil \\
\hline SD & QLP stw. & 1_B2 & 0.6 & 14 & $\min \mathrm{PT}$ & 11000 & 2600 & 26000 & 10000 & 450 & 12 & 17 & ND & 520 & 640 & 70 & \\
\hline
\end{tabular}

Averages (Av.; $\mu \mathrm{g} / \mathrm{g}$ ) and standard deviations (SD; 1 sigma) of elements in Cu-stage (quartz stockwork veins). Salinities (Sal.; equivalent NaCl wt \%) and homogenization temperatures $\left(\mathrm{T}_{h}\right)$ of the assemblages were obtained from microthermometry (Bodnar and Vityk, 1994) and pressures of entrapment were estimated (Driesner and Heinrich, 2007). "Deep stw.", “Cu-vein”, and "QLP stw” represent low Cu grade quartz stockwork veins, high Cu grade quartz stockwork vein, and late-stage (post-QLP) quartz stockwork vein, respectively. Boiling assemblages "boil" allow direct calculation "real PT" of pressures, whereas brine-only "no-boil" assemblages provide minimum estimation "min PT" of pressures. "ND" represents values not determined. 
Table 3. Composition of Mo-stage (quartz-molybdenite veins) brine inclusion assemblages

\begin{tabular}{|c|c|c|c|c|c|c|c|c|c|c|c|c|c|c|c|c|c|}
\hline$\mu \mathrm{g} / \mathrm{g}$ & Location & Sample\# & Sal. & $\operatorname{Th}\left({ }^{\circ} \mathrm{C}\right)$ & $\mathbf{P}($ bar $)$ & ${ }^{23} \mathrm{Na}$ & ${ }^{32} \mathrm{~S}$ & ${ }^{39} \mathrm{~K}$ & ${ }^{57} \mathrm{Fe}$ & ${ }^{65} \mathrm{Cu}$ & ${ }^{75} \mathrm{As}$ & ${ }^{133} \mathrm{Cs}$ & ${ }^{197} \mathrm{Au}$ & ${ }^{208} \mathrm{~Pb}$ & ${ }^{66} \mathrm{Zn}$ & ${ }^{95} \mathrm{Mo}$ & mark \\
\hline Av. & center & D50-2155 & 41.4 & 469 & 362 & 110000 & 5600 & 110000 & 48000 & 300 & 39 & 54 & 0.16 & 3100 & 2800 & 160.0 & boil \\
\hline SD & deep Moly & A 02 & 0.4 & 22 & realPT & 5900 & 1200 & 15000 & 6100 & 74 & 5 & 9 & 0.07 & 490 & 520 & 100.0 & \\
\hline Av. & center & D50-2155 & 42.0 & 426 & 245 & 120000 & 6100 & 100000 & 41000 & 360 & 29 & 46 & 0.41 & 3200 & 2500 & 380.0 & no-boil \\
\hline SD & deep Moly & $\mathrm{A} 03$ & 1.0 & 21 & $\operatorname{minPT}$ & 2800 & 1300 & 7300 & 4700 & 100 & 10 & 5 & 0.10 & 350 & 290 & 8.6 & \\
\hline Av. & center & D50-2155 & 40.0 & 426 & 251 & 110000 & 5700 & 99000 & 49000 & 250 & 30 & 48 & 0.26 & 3000 & 2700 & 75.0 & no-boil \\
\hline SD & deep Moly & A04 & 0.5 & 15 & $\operatorname{minPT}$ & 710 & 500 & 1800 & 1900 & 47 & 5 & 6 & ND & 59 & 91 & 5.9 & \\
\hline Av. & center & D50-2155 & 42.0 & 498 & 447 & 120000 & 3700 & 100000 & 44000 & 750 & 35 & 46 & 0.21 & 2900 & 2400 & 130.0 & boil \\
\hline SD & deep Moly & A05 & 0.8 & 8 & realPT & 1300 & 2300 & 3300 & 2300 & 740 & 11 & 5 & 0.17 & 210 & 360 & 110.0 & \\
\hline Av. & center & D50-2155 & 40.2 & 404 & 205 & 120000 & 7200 & 97000 & 39000 & 920 & 35 & 39 & 0.11 & 2600 & 2300 & 87.0 & boil \\
\hline SD & deep Moly & B 01 & 1.7 & 28 & realPT & 1400 & 510 & 3400 & 4300 & 600 & 4 & 3 & 0.03 & 340 & 390 & 14.0 & \\
\hline Av. & center & D50-2155 & 41.5 & 412 & 217 & 130000 & 7100 & 81000 & 35000 & 720 & 27 & 38 & 0.55 & 2600 & 1900 & 77.0 & boil \\
\hline SD & deep Moly & В 02 & 0.9 & 17 & realPT & 9200 & 1800 & 24000 & 18000 & 890 & 10 & 8 & 0.41 & 450 & 760 & 37.0 & \\
\hline Av. & center & D50-2155 & 36.9 & 416 & 240 & 110000 & 7100 & 88000 & 39000 & 1200 & 28 & 37 & 0.30 & 2600 & 2400 & 97.0 & boil \\
\hline SD & deep Moly & В 03 & 0.9 & 21 & realPT & 2400 & 2800 & 6800 & 5900 & 1600 & 3 & 3 & 0.03 & 360 & 290 & 33.0 & \\
\hline Av. & center & D50-2155 & 42.3 & 403 & 197 & 130000 & 3200 & 97000 & 39000 & 1500 & 24 & 29 & 0.26 & 2800 & 1800 & 150.0 & boil \\
\hline SD & deep Moly & B04 & 1.4 & 33 & realPT & 6200 & 1900 & 15000 & 18000 & 980 & 10 & 12 & 0.26 & 380 & 970 & 120.0 & \\
\hline Av. & center & D50-2155 & 40.7 & 399 & 193 & 110000 & 7900 & 110000 & 51000 & 340 & 30 & 55 & 0.22 & 3000 & 3200 & 100.0 & boil \\
\hline SD & deep Moly & B06 & 0.7 & 40 & realPT & 3200 & 2800 & 7300 & 14000 & 140 & 9 & 19 & 0.00 & 350 & 1000 & 64.0 & \\
\hline Av. & center & D50-2379 & 41.1 & 295 & ND & 14000 & 7600 & 68000 & 33000 & 7900 & 74 & 32 & 1.90 & 2500 & 2500 & 360.0 & boil \\
\hline SD & deep Moly & 1_B3 & 1.5 & 6 & & 1200 & 2100 & 4900 & 1900 & 1900 & 59 & 8 & 0.10 & 240 & 870 & 420.0 & \\
\hline Av. & center & D50-2379 & 40.5 & 273 & ND & 130000 & 7100 & 64000 & 11000 & 1200 & 240 & 47 & ND & 6200 & 5800 & ND & boil \\
\hline SD & deep Moly & 1_TP1 & 2.1 & 33 & & 3900 & 4400 & 10000 & 3900 & 740 & 23 & 9 & ND & 1800 & 1000 & ND & \\
\hline Av. & center & D50-2379 & 50.6 & 275 & ND & 170000 & 4700 & 71000 & 44000 & 4600 & 64 & 51 & 1.70 & 3300 & 3400 & ND & boil \\
\hline SD & deep Moly & 1_B5 & 0.8 & 7 & & 5900 & 240 & 15000 & 5300 & 2300 & 8 & 5 & ND & 370 & 280 & ND & \\
\hline Av. & center & D50-2379 & 44.0 & 289 & ND & 140000 & 5700 & 76000 & 43000 & 5700 & 48 & 44 & 0.81 & 3300 & 3200 & 150.0 & boil \\
\hline SD & deep Moly & 1_TP4 & 0.3 & 12 & & 9500 & 2800 & 24000 & 7800 & 3900 & 18 & 15 & 0.60 & 850 & 1000 & 190.0 & \\
\hline Av. & center & $\mathrm{JH} 2$ & 45.2 & 475 & 357 & 120000 & 9200 & 73000 & 53000 & 3600 & 55 & 43 & 2.30 & 3300 & 4000 & 47.0 & no-boil \\
\hline SD & Mo-vein & b1e & 1.0 & ND & $\operatorname{minPT}$ & 9000 & 2500 & 13000 & 8200 & 1400 & 37 & 10 & 2.30 & 240 & 1400 & 20.0 & \\
\hline Av. & center & $\mathrm{JH} 2$ & 40.9 & 413 & 220 & 99000 & 21000 & 98000 & 53000 & 16000 & 25 & 39 & 0.68 & 3400 & 3000 & 380.0 & boil \\
\hline SD & Mo-vein & b1b & 1.8 & 12 & realPT & 18000 & 4700 & 36000 & 17000 & 6300 & 10 & 19 & 0.30 & 1100 & 1100 & 170.0 & \\
\hline Av. & center & $\mathrm{JH} 2$ & 47.7 & 414 & 200 & 120000 & 14000 & 90000 & 62000 & 10000 & 30 & 57 & 0.26 & 3500 & 3800 & 120.0 & boil \\
\hline SD & Mo-vein & b1a & 1.1 & 11 & realPT & 4700 & 4400 & 13000 & 5900 & 4000 & 6 & 5 & 0.02 & 340 & 840 & 47.0 & \\
\hline
\end{tabular}




\begin{tabular}{|c|c|c|c|c|c|c|c|c|c|c|c|c|c|c|c|c|c|}
\hline Av. & center & JH2 & 45.4 & 399 & 179 & 100000 & 25000 & 100000 & 65000 & 24000 & 12 & 39 & 4.60 & 4400 & 4500 & 360.0 & no-boil \\
\hline SD & Mo-vein & b1d & 1.7 & 12 & $\operatorname{minPT}$ & 22000 & 11000 & 53000 & 14000 & 4200 & 0 & 15 & 6.40 & 2800 & 3300 & 220.0 & \\
\hline Av. & center & JH2 & 41.3 & 572 & 691 & 110000 & 2900 & 86000 & 42000 & 910 & 34 & 39 & 0.25 & 2400 & 1800 & 38.0 & no-boil \\
\hline SD & Mo-vein & b1c & 1.0 & 11 & $\operatorname{minPT}$ & 6100 & 1100 & 12000 & 4300 & 150 & 8 & 7 & 0.12 & 230 & 380 & 18.0 & \\
\hline Av. & center & JH2 & 46.4 & 415 & 207 & 120000 & 12000 & 83000 & 52000 & 5700 & 56 & 46 & 0.71 & 3500 & 5000 & 93.0 & boil \\
\hline SD & Mo-vein & b2b & 3.9 & 21 & realPT & 13000 & 8200 & 19000 & 18000 & 4700 & 23 & 14 & 0.29 & 930 & 1800 & 110.0 & \\
\hline Av. & center & JH2 & 43.4 & 469 & 351 & 100000 & 11000 & 100000 & 55000 & 16000 & 20 & 33 & 0.31 & 2800 & 2300 & 350.0 & boil \\
\hline SD & Mo-vein & b2c & 0.3 & 4 & realPT & 3800 & 1900 & 8600 & 4100 & 1700 & 7 & 7 & 0.11 & 660 & 350 & 84.0 & \\
\hline Av. & center & JH2 & 42.1 & 428 & 249 & 100000 & 11000 & 85000 & 58000 & 13000 & 28 & 27 & 1.50 & 2800 & 2900 & 360.0 & boil \\
\hline SD & Mo-vein & b2e & 0.4 & 11 & realPT & 8900 & 1700 & 13000 & 8400 & 1900 & 13 & 7 & 2.00 & 250 & 760 & 59.0 & \\
\hline Av. & center & JH2 & 47.7 & 407 & 187 & 120000 & 6800 & 100000 & 51000 & 1000 & 23 & 44 & 0.19 & 3200 & 3000 & 72.0 & no-boil \\
\hline SD & Mo-vein & b2d & 0.2 & 2 & $\operatorname{minPT}$ & 4600 & 1200 & 8000 & 5100 & 630 & 6 & 10 & 0.11 & 170 & 520 & 8.7 & \\
\hline Av. & center & $\mathrm{JH} 2$ & 39.3 & 542 & 609 & 100000 & 8300 & 89000 & 36000 & 530 & 22 & 25 & 0.42 & 2100 & 1700 & 25.0 & boil \\
\hline SD & Mo-vein & b2f & 0.3 & 30 & realPT & 9800 & 3700 & 9700 & 15000 & 270 & 11 & 12 & 0.49 & 570 & 830 & 7.2 & \\
\hline Av. & center & JH2 & 39.3 & 325 & 84 & 100000 & 7400 & 69000 & 41000 & 6900 & 18 & 29 & 1.20 & 2600 & 2000 & 83.0 & no-boil \\
\hline SD & Mo-vein & a1-1 & 2.7 & ND & $\operatorname{minPT}$ & 6300 & 1800 & 10000 & 8100 & 4600 & 3 & 12 & 0.24 & 430 & 670 & 46.0 & \\
\hline Av. & center & JH2 & 42.0 & ND & ND & 110000 & 3600 & 83000 & 41000 & 390 & 45 & 33 & 0.29 & 2600 & 2100 & 5.4 & no-boil \\
\hline SD & Mo-vein & a1-2 & 1.8 & ND & & 10000 & 510 & 15000 & 11000 & 260 & 21 & 10 & 0.17 & 540 & 680 & 1.3 & \\
\hline Av. & center & $\mathrm{JH} 2$ & 39.3 & 330 & 89 & 100000 & 6800 & 82000 & 44000 & 310 & 25 & 42 & 0.37 & 2800 & 2700 & 33.0 & no-boil \\
\hline SD & Mo-vein & a1-3 & 0.8 & 8 & $\operatorname{minPT}$ & 9200 & 2600 & 16000 & 6200 & 190 & 3 & 12 & ND & 680 & 900 & 13.0 & \\
\hline Av. & center & $\mathrm{JH} 2$ & 44.4 & 475 & 362 & 110000 & 8500 & 100000 & 53000 & 780 & 27 & 48 & 0.31 & 2900 & 2800 & 140.0 & no-boil \\
\hline SD & Mo-vein & a5-2 & 0.7 & 7 & $\operatorname{minPT}$ & 6200 & 1900 & 15000 & 3600 & 150 & 3 & 4 & 0.22 & 780 & 360 & 21.0 & \\
\hline Av. & center & JH2 & 42.1 & 580 & 709 & 110000 & 6800 & 96000 & 40000 & 200 & 33 & 36 & 0.40 & 2700 & 2800 & 1.8 & boil \\
\hline SD & Mo-vein & a5-3 & 0.1 & 30 & realPT & 2600 & 2200 & 3600 & 4200 & 34 & 2 & 5 & 0.17 & 400 & 440 & 1.0 & \\
\hline Av. & center & $\mathrm{JH} 2$ & 46.2 & 411 & 200 & 110000 & 23000 & 100000 & 58000 & 25000 & 24 & 47 & 0.16 & 3100 & 3200 & 88.0 & boil \\
\hline SD & Mo-vein & a6-1 & 1.0 & 4 & realPT & 1600 & 2300 & 8700 & 6100 & 2500 & 7 & 3 & ND & 260 & 460 & 50.0 & \\
\hline Av. & center & JH2 & 49.3 & 431 & 227 & 110000 & 23000 & 120000 & 62000 & 27000 & 16 & 47 & 0.64 & 3300 & 3200 & 26.0 & no-boil \\
\hline SD & Mo-vein & a6-2 & 0.5 & ND & $\operatorname{minPT}$ & 4800 & 4700 & 7200 & 4700 & 3000 & 3 & 6 & 0.49 & 380 & 450 & 5.9 & \\
\hline Av. & center & $\mathrm{JH} 2$ & 46.8 & 425 & 225 & 110000 & 11000 & 110000 & 57000 & 520 & 22 & 42 & 0.25 & 3100 & 3200 & 110.0 & boil \\
\hline SD & Mo-vein & a6-3 & 1.5 & 3 & realPT & 2800 & 3700 & 6300 & 3600 & 190 & 10 & 6 & 0.12 & 530 & 750 & 13.0 & \\
\hline Av. & center & $\mathrm{JH} 2$ & 49.9 & 429 & 221 & 130000 & 17000 & 110000 & 52000 & 16000 & 13 & 43 & 0.29 & 2900 & 2900 & 20.0 & boil \\
\hline SD & Mo-vein & a7 & 0.4 & ND & realPT & 7400 & 3100 & 15000 & 6700 & 9500 & 4 & 4 & 0.12 & 220 & 1000 & 20.0 & \\
\hline Av. & center & $\mathrm{JH} 2$ & 45.8 & 431 & 242 & 100000 & 24000 & 120000 & 59000 & 2700 & 26 & 60 & 0.45 & 4000 & 4100 & 110.0 & boil \\
\hline SD & Mo-vein & a7-1 & 1.4 & 3 & realPT & 11000 & 9700 & 21000 & 7400 & 3300 & 8 & 13 & 0.26 & 870 & 1200 & 45.0 & \\
\hline Av. & center & JH2 & 43.4 & 427 & 243 & 88000 & 5300 & 85000 & 33000 & 220 & 23 & 39 & 0.14 & 2400 & 2400 & 130.0 & boil \\
\hline
\end{tabular}




\begin{tabular}{|c|c|c|c|c|c|c|c|c|c|c|c|c|c|c|c|c|c|}
\hline SD & Mo-vein & a7-3 & 0.8 & 16 & realPT & 7500 & 2000 & 9500 & 8600 & 150 & 4 & 10 & 0.07 & 370 & 550 & 41.0 & \\
\hline Av. & center & $\mathrm{JH} 2$ & 39.0 & 580 & 742 & 97000 & 5100 & 85000 & 52000 & 190 & 27 & 37 & 0.20 & 2800 & 2300 & 51.0 & no-boil \\
\hline SD & Mo-vein & a7-4 & 0.5 & $\min T$ & $\operatorname{minPT}$ & 4700 & 1600 & 3000 & 8600 & 62 & 6 & 8 & 0.02 & 310 & 450 & 10.0 & \\
\hline Av. & center & $\mathrm{JH} 2$ & 59.4 & ND & ND & 160000 & 6600 & 110000 & 50000 & 580 & 30 & 61 & 0.09 & 4000 & 3400 & 18.0 & no-boil \\
\hline SD & Mo-vein & a7-5 & 2.0 & ND & & 6000 & 1300 & 10000 & 5700 & 78 & 4 & 11 & 0.05 & 380 & 320 & 1.5 & \\
\hline Av. & center & JH2 & 39.7 & 526 & 553 & 100000 & 4500 & 83000 & 45000 & 540 & 29 & 42 & 0.07 & 2500 & 2400 & 7.2 & boil \\
\hline SD & Mo-vein & a7-6 & ND & 11 & realPT & 5400 & 940 & 8000 & 5200 & 190 & 4 & 4 & 0.03 & 430 & 630 & 3.2 & \\
\hline Av. & center & JH2 & 41.2 & 492 & 434 & 120000 & 6000 & 79000 & 39000 & 1200 & 51 & 42 & 1.30 & 3800 & ND & 88.0 & boil \\
\hline SD & Mo-vein & B_BT2 & 2.1 & & realPT & 10000 & 3200 & 11000 & 13000 & 950 & 31 & 7 & 1.00 & 970 & $\mathrm{ND}$ & 80.0 & \\
\hline Av. & periphery & D511-3120 & 40.2 & ND & ND & 120000 & 13000 & 95000 & 60000 & 8800 & 59 & 38 & ND & 2900 & 3600 & 30.0 & no-boil \\
\hline SD & Mo-vein & 1_B2 & 0.6 & ND & & 1500 & 2600 & 3900 & 4600 & 8200 & 19 & 9 & ND & 190 & 310 & 4.9 & \\
\hline Av. & periphery & D511-3120 & 37.5 & 381 & 166 & 110000 & 8800 & 82000 & 38000 & 8100 & 24 & 35 & ND & 2700 & 2600 & 31.0 & no-boil \\
\hline SD & Mo-vein & 1_B3 & 0.5 & 5 & $\min P T$ & 3300 & 4200 & 9600 & 1600 & 6000 & 12 & 10 & ND & 490 & 340 & 30.0 & \\
\hline Av. & periphery & D511-3120 & 35.3 & 360 & 136 & 100000 & 12000 & 90000 & 40000 & 9100 & 35 & 32 & 3.60 & 2500 & 2900 & 76.0 & boil \\
\hline SD & Mo-vein & 1_TP4 & 1.3 & 17 & realPT & 3500 & 4500 & 9200 & 1600 & 1100 & 22 & 7 & 0.24 & 140 & 380 & 27.0 & \\
\hline Av. & periphery & D511-3120 & 42.0 & 319 & ND & 120000 & 17000 & 94000 & 46000 & 11000 & 82 & 51 & 1.60 & 2700 & 2900 & 130.0 & no-boil \\
\hline SD & Mo-vein & 1_B5 & 0.1 & 13 & & 86 & 11000 & 340 & 7500 & 860 & 66 & 18 & ND & 250 & 67 & 42.0 & \\
\hline Av. & periphery & D511-3120 & 37.1 & 377 & 160 & 110000 & 10000 & 80000 & 43000 & 10000 & 36 & 36 & ND & 2500 & 2800 & 120.0 & boil \\
\hline SD & Mo-vein & 1_TP6(b) & 0.9 & 20 & realPT & 4200 & 1200 & 10000 & 5000 & 3200 & 6 & 8 & ND & 110 & 730 & 65.0 & \\
\hline Av. & periphery & D511-3120 & 37.1 & ND & ND & 110000 & 16000 & 90000 & 56000 & 10000 & 28 & 63 & 13.00 & 2500 & 5400 & 76.0 & no-boil \\
\hline SD & Mo-vein & 1_B7 & ND & ND & & 13000 & 4500 & 30000 & 22000 & 2600 & ND & 43 & ND & 460 & 4100 & 18.0 & \\
\hline Av. & center & $\mathrm{JH} 4$ & 39.4 & ND & ND & 87000 & 7100 & 55000 & 29000 & 1200 & ND & ND & ND & 1900 & ND & 1.7 & no-boil \\
\hline SD & Mo-vein & a_B1 & ND & ND & & 5700 & 4900 & 4800 & 2300 & 470 & ND & ND & ND & 410 & ND & ND & \\
\hline Av. & center & JH4 & 47.2 & ND & ND & 100000 & 4300 & 68000 & 37000 & 830 & ND & ND & ND & 2100 & ND & 6.5 & no-boil \\
\hline SD & Mo-vein & a_B2 & ND & ND & & 6600 & 3400 & 10000 & 2200 & 480 & ND & ND & ND & 270 & ND & 3.2 & \\
\hline Av. & center & $\mathrm{JH} 4$ & 39.1 & ND & ND & 81000 & 3000 & 58000 & 33000 & 590 & ND & ND & ND & 1800 & ND & 1.9 & no-boil \\
\hline SD & Mo-vein & a_B3 & 1.3 & ND & & 2900 & 2300 & 2000 & 1200 & 390 & ND & ND & ND & 140 & ND & 0.3 & \\
\hline Av. & center & JH4 & 41.5 & ND & ND & 130000 & 3600 & 88000 & 48000 & 1700 & ND & 40 & ND & 2900 & ND & 8.9 & no-boil \\
\hline SD & Mo-vein & a_B4 & ND & ND & & 2800 & 770 & 7500 & 7200 & 350 & ND & 5 & ND & 250 & ND & 9.1 & \\
\hline Av. & center & $\mathrm{JH} 4$ & 36.8 & 468 & 383 & 110000 & 3300 & 82000 & 44000 & 1000 & 44 & 44 & ND & 2900 & 3100 & 36.0 & boil \\
\hline SD & Mo-vein & $b_{-}$TP1 & 0.4 & 2 & realPT & 3200 & 2000 & 8200 & 1800 & 730 & 17 & 2 & ND & 530 & 460 & 10.0 & \\
\hline Av. & center & $\mathrm{JH} 4$ & 45.7 & 395 & 171 & 130000 & 3200 & 100000 & 65000 & 1000 & 75 & 58 & 0.84 & 3600 & 2700 & 3.2 & no-boil \\
\hline SD & Mo-vein & b_B2 & 0.2 & 2 & $\min \mathrm{PT}$ & 6600 & 840 & 16000 & 9900 & 220 & 11 & 17 & ND & 740 & 510 & 0.4 & \\
\hline Av. & center & $\mathrm{JH} 4$ & 39.5 & 463 & 354 & 120000 & 8500 & 90000 & 48000 & 14000 & 21 & 46 & 1.40 & 3100 & 2600 & 69.0 & no-boil \\
\hline
\end{tabular}




\begin{tabular}{|c|c|c|c|c|c|c|c|c|c|c|c|c|c|c|c|c|c|}
\hline SD & Mo-vein & b_B3 & 1.0 & 5 & $\min P T$ & 8700 & 3000 & 22000 & 4800 & 4000 & 7 & 12 & 0.65 & 900 & 440 & 23.0 & \\
\hline Av. & center & JH4 & 42.3 & 388 & 168 & 130000 & 9300 & 92000 & 50000 & 1200 & 25 & 49 & ND & 3400 & 2900 & 13.0 & no-boil \\
\hline SD & Mo-vein & b_B4 & 0.6 & 1 & $\min P T$ & 5000 & 2700 & 11000 & 7300 & 450 & ND & 0 & ND & 880 & 890 & ND & \\
\hline Av. & center & JH4 & 39.0 & 475 & 394 & 110000 & 5500 & 95000 & 34000 & 780 & 140 & 51 & 0.89 & 3500 & 3200 & 3.4 & no-boil \\
\hline SD & Mo-vein & b_B6 & ND & ND & $\min P T$ & 10000 & 1100 & 25000 & 8500 & 550 & 57 & 19 & 0.03 & 490 & 970 & $\mathrm{ND}$ & \\
\hline Av. & center & $4990-1470$ & 41.8 & 413 & 217 & 120000 & 7500 & 84000 & 34000 & 2200 & 24 & 34 & 0 & 2500 & 2300 & 7.5 & no-boil \\
\hline SD & Mo-vein & 2_B1 & 0.8 & 9 & $\min P T$ & 5300 & 4900 & 13000 & 8200 & 1300 & 7 & 12 & 0 & 390 & 880 & 3.0 & \\
\hline Av. & center & $4990-1470$ & 54.3 & 343 & ND & 160000 & 2900 & 110000 & 46000 & 490 & 78 & 50 & 1 & 3500 & 2700 & 16.0 & no-boil \\
\hline SD & Mo-vein & 2_B2 & 0.1 & 5 & & 4900 & 380 & 12000 & 7100 & 200 & 55 & 6 & 0 & 140 & 240 & 10.0 & \\
\hline Av. & center & $4990-1470$ & 51.0 & 362 & ND & 150000 & 4000 & 98000 & 45000 & 5300 & 50 & 50 & 1 & 3300 & 3000 & 5.6 & no-boil \\
\hline SD & Mo-vein & 2_B3 & 0.6 & ND & & 3000 & 1100 & 6500 & 9600 & 2500 & 26 & 11 & 1 & 420 & 750 & 1.1 & \\
\hline Av. & center & $4990-1470$ & 50.7 & ND & ND & 150000 & 3800 & 110000 & 47000 & 2300 & 61 & 57 & 0 & 3400 & 3200 & 5.8 & no-boil \\
\hline SD & Mo-vein & 2_B4 & ND & ND & & 4800 & 1700 & 11000 & 7400 & 1000 & 32 & 10 & 0 & 540 & 610 & 4.0 & \\
\hline
\end{tabular}

"deep moly", and "Mo-vein" represent low grade quartz-molybdenite veins, and high grade quartz-molybdenite veins, respectively. 
(A) Mo-stage, barren core (D50-2155) quartz vein

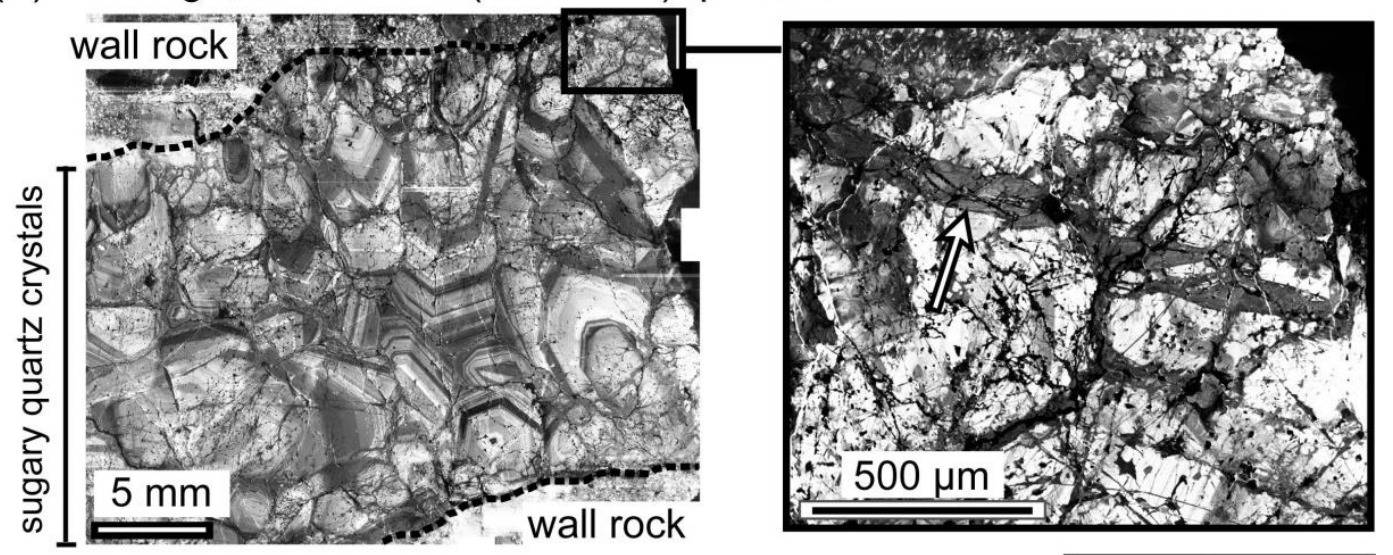

(B) Mo-stage, high-grade center (JH2) quartz crystal

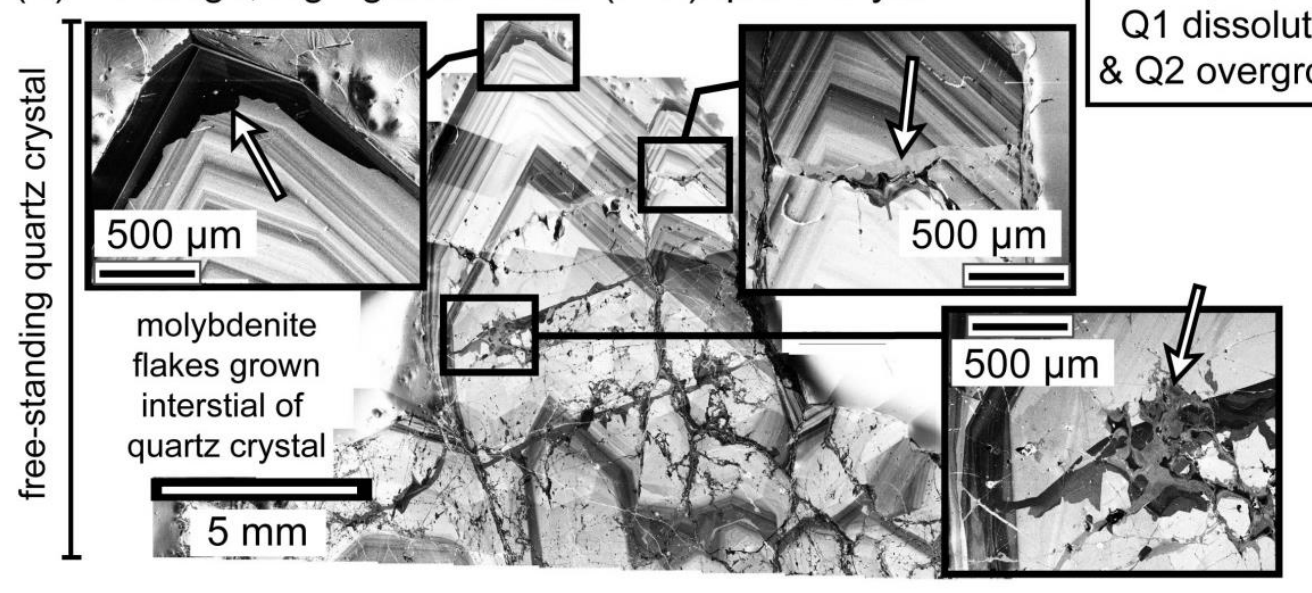

(C) Mo-stage, high-grade center (JH4) quartz vein

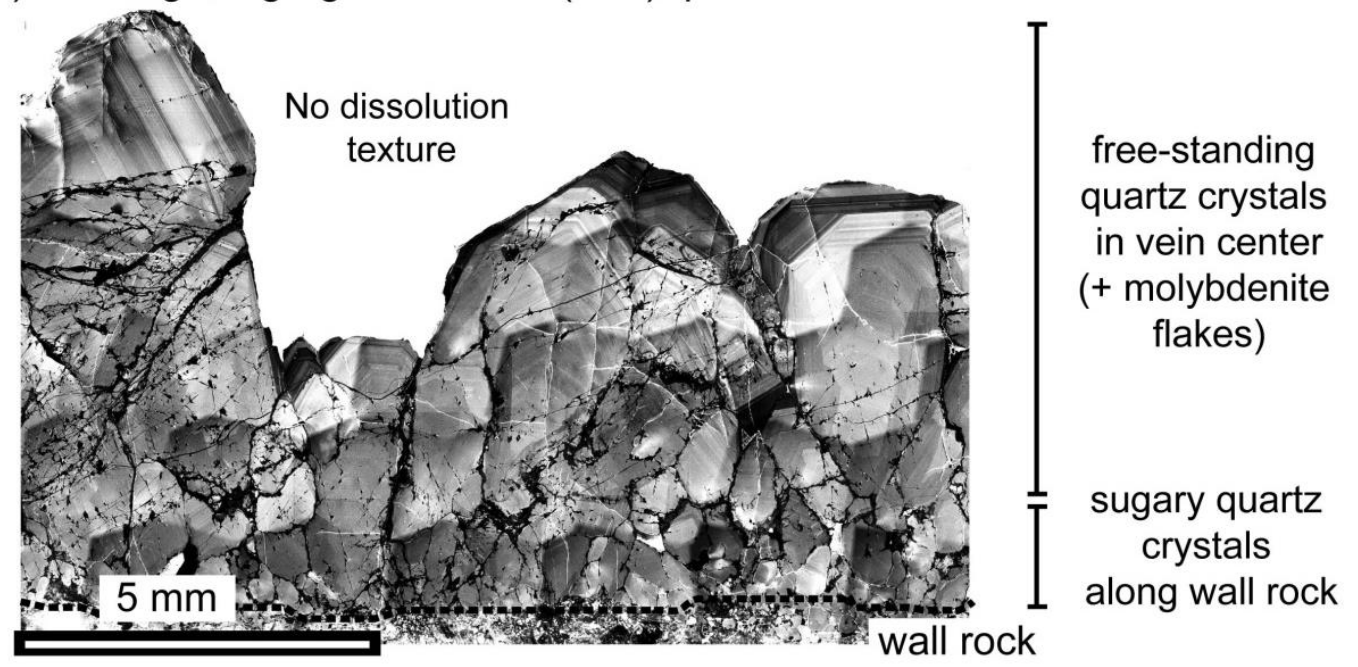

Figure 4. Cathodo-luminescience (CL) images of quartz-molybdenite veins from the low-grade (D50-2155) and high-grade $(\mathrm{JH} 2, \mathrm{JH} 4)$ zones. These quartz veins have both euhedral sugary quartz (A) and euhedral freestanding quartz crystals (B, C). Textures of early-generated quartz dissolution (Q1) and second generation quartz overgrowth (Q2) are prominent in the low-grade (A) and high-grade (B) quartz-molybdenite veins. Some quartz-molybdenite veins, however, do not show quartz dissolution textures (C). 
In-situ analyses of single fluid inclusions were conducted by laser ablation inductively coupled mass spectrometry (LA-ICP-MS). A beam-homogenized $193 \mathrm{~nm}$ ArF Excimer laser ablation system (ETH prototype, similar to Geolas system; Günther et al., 1997) was connected to a quadruple ICP-MS (Perkin Elmer Elan 6100 DRC) optimized for the detection of sulfur as well as metals (Guillong et al., 2008a; Seo et al., 2011). By selection of a reduced set of isotopes to be measured $\left({ }^{23} \mathrm{Na},{ }^{29} \mathrm{Si},{ }^{32} \mathrm{~S},{ }^{39} \mathrm{~K},{ }^{55} \mathrm{Mn},{ }^{57} \mathrm{Fe},{ }^{65} \mathrm{Cu}, 66 \mathrm{Zn},{ }^{75} \mathrm{As},{ }^{85} \mathrm{Rb},{ }^{88} \mathrm{Sr}, 95 \mathrm{Mo}\right.$, ${ }^{133} \mathrm{Cs},{ }^{137} \mathrm{Ba},{ }^{197} \mathrm{Au}$ and ${ }^{208} \mathrm{~Pb}$ ) and applying an increased dwell time on selected el ements (i.e., $50 \mathrm{~ms}$ for ${ }^{197} \mathrm{Au},{ }^{95} \mathrm{Mo}, 20 \mathrm{~ms}$ for ${ }^{32} \mathrm{~S}$, and $10 \mathrm{~ms}$ for other elements), we reduced the noise of the instrumental backgrounds to improve limits of detection while maintaining the necessary time resolution for representative detection of inclusion signals. We also analyzed ${ }^{55} \mathrm{Mn}, 66 \mathrm{Zn},{ }^{85} \mathrm{Rb},{ }^{88} \mathrm{Sr}$ and ${ }^{137} \mathrm{Ba}$ in some brine inclusion assemblages in order to focus on $\mathrm{Fe} / \mathrm{Mn}$ ratios. A small amount of $\mathrm{H}_{2}$ gas ( $5 \mathrm{ml} / \mathrm{min}$.) added to the He carrier gas increases the sensitivity of selective elements such as $\mathrm{Cu}, \mathrm{Au}$, and Mo (Guillong and Heinrich, 2007). Energy densities between 30 and $40 \mathrm{~J} / \mathrm{cm}^{2}$ on the sample surface with $10 \mathrm{~Hz}$ pulse frequency allowed controlled sampling of most quartz-hosted fluid inclusions. An Iris aperture was gradually opened during analysis to a spot size allowing complete excavation of the fluid inclusion without the loss of daughter crystals. Quantification followed the data reduction procedures of Günther et al. (1998) and Heinrich et al. (2003) using the SILLS software (Guillong et al., 2008b) for data reduction. Since salinity measurements for the vapor-rich inclusions are difficult, we used element ratios for interpretations of the vapor phase fluids.

We also analyzed some of the intermediate-density and vapor inclusions in quartz veins (A30-3816 and D211-19 in Cu-stage, and D50-2155, 2379, A57-5931, JH2, and JH4 in Mostage) by using laser Raman microspectroscopy in ETH Zurich and University of Bern in order to check daughter minerals, and to compare the gas compositions and ratios of $\mathrm{CO}_{2}$ and $\mathrm{CH}_{4}$.

\section{Results}

\section{Pressure and temperature}

Salinities ( $\mathrm{NaCl}$ equivalent wt \%) of brine inclusion assemblages were estimated from halite dissolution temperature s (Bodnar and Vityk, 1994). Some inclusions homogenized by halite dissolution, but most by bubble disappearance, providing estimates of minimum temperature and pressures in the case of brine-only assemblages (Fig. 5). Microthermometric data from brine inclusions in boiling assemblages yield estimates of absolute temperature and pressure of entrapment. True pressures are probably somewhat higher than indicated by the $\mathrm{NaCl}-\mathrm{H}_{2} \mathrm{O}$ model system (Bodnar and Vityk, 1994; Driesner and Heinrich, 2007) because of the presence of minor $\mathrm{CO}_{2}$, but also affected by $\mathrm{H}_{2} \mathrm{~S}$ and other cations indicated by Raman and ICP-MS analyses (Fig. 6). Consistency of homogenization temperatures within brine inclusion as semblages are generally $\pm 40^{\circ} \mathrm{C}$ or better (Table 2,3 and Fig. 5).

The trapping temperature and calculated pressure of the $\mathrm{Cu}$-stage brines obtained from boiling assemblages in low-grade and high-grade quartz stockwork vein samples are from $320 \pm 15$ to $430 \pm 7{ }^{\circ} \mathrm{C}$ (average $\pm 1 \sigma$ of fluid inclusion assemblage) and from 90 to 260 bars (Fig. 5), consistent with the previously reported conditions of $\mathrm{Cu}-\mathrm{Au}$ precipitation at Bingham Canyon (Landtwing et al., 2005; Landtwing et al., 2010). The temperatures and pressures of Mo-stage boiling assemblages extend to significantly higher values than those from the $\mathrm{Cu}$-Au stage fluids, with a maximum of $580 \pm 30^{\circ} \mathrm{C}$ (Fig. 5) implying a pressure of 710 bar. Brines from boiling assemblages in low Mo grade quartz-molybdenite veins are trapped at temperature from $400 \pm 40$ to $500 \pm 8{ }^{\circ} \mathrm{C}$ indicating pressures of at least 200- 
450 bars (Fig. 5), but possibly higher during the trapping of the predominant intermediatedensity inclusions in these deep samples within the single-phase fluid stability field. Brines from boiling assemblages in high Mo grade quartz-molybdenite veins are trapped at temperatures from $360 \pm 20$ to $580 \pm 30{ }^{\circ} \mathrm{C}$ indicating pressures of $140-710$ bars, comparable ranges to low Mo grade quartz-molybdenite vein samples (Fig. 5). Maximum pressures around 710 bars are consistent with tentative estimations for lithostatic pressure conditions in the barren core during $\mathrm{Cu}-\mathrm{Au}$ mineralization (Landtwing et al., 2010). Temperatures obtained from boiling ass emblages vary from 320 to $430{ }^{\circ} \mathrm{C}$ in the $\mathrm{Cu}$-stage fluids, but show a much greater variation from 270 to $580{ }^{\circ} \mathrm{C}$ in the Mo-stage fluids, extending to higher temperatures (Fig. 5).

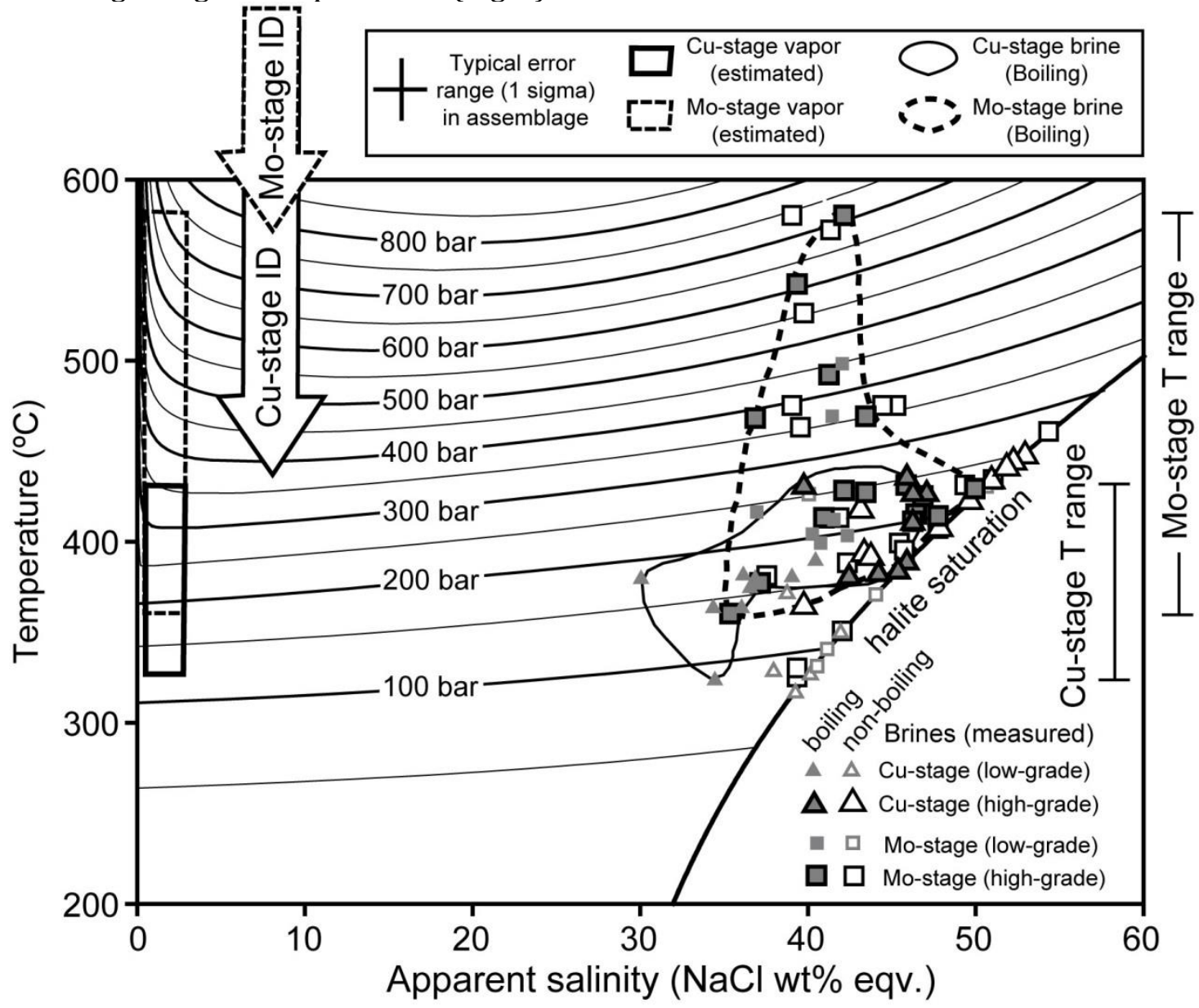

Figure 5. Plots of measured homogenization temperatures $\left(\mathrm{Th} ;{ }^{\circ} \mathrm{C}\right)$ vs. apparent salinities $(\mathrm{NaCl}$ wt $\%$ equivalent) in the brine inclusion assemblages of early $\mathrm{Cu}$-stage (quartz stockwork veins) and late Mo-stage (quartzmolybdenite veins). Contours of pressure (bar) were calculated based on the $\mathrm{H}_{2} \mathrm{O}-\mathrm{NaCl}$ system (Driesner and Heinrich, 2007). Filled symbols represent "boiling assemblage" direct estimations of temperature; Open symbols represent brine-only assemblages of minimum temperature estimations. Among the boiling assemblages in the $\mathrm{Cu}$-stage, brine salinities are higher in the high-grade sample than in the low-grade sample. Comparable brine salinities were found in the Mo-stage samples. Homogenization temperatures in Mo-stage fluids were found to be higher than that in $\mathrm{Cu}$-stage fluids. Typical error ranges in the assemblages are $\pm 3 \mathrm{wt} \%$ and $\pm 20^{\circ} \mathrm{C}$.

\section{Raman spectroscopy and redox conditions}

Gas ratios of $\mathrm{CH}_{4} / \mathrm{CO}_{2}$ in the intermediate-density and vapor-rich inclusions in quartz stockwork veins (A30-3816 and D211-19) and quartz-molybdenite veins (D50-2155, 2379, A57-5931, JH2, and JH4) were analyzed by Raman spectroscopy at room temperature to 
detect trends indicative of redox evolution. However, the low Raman cross-section of $\mathrm{CH}_{4}$ (Burke, 2001) prevented detection of $\mathrm{CH}_{4}$ in any intermediate-density or vapor inclusions (Fig. 6). Calculated detection limits for the vapor inclusions in Bingham Canyon deposit, based on the peak areas and background variation of the Raman spectrum and instrumental sensitivities of gas species (Burke, 2001), indicate a maximum $\mathrm{CH}_{4} / \mathrm{CO}_{2}$ molar ratios of 0.002 .

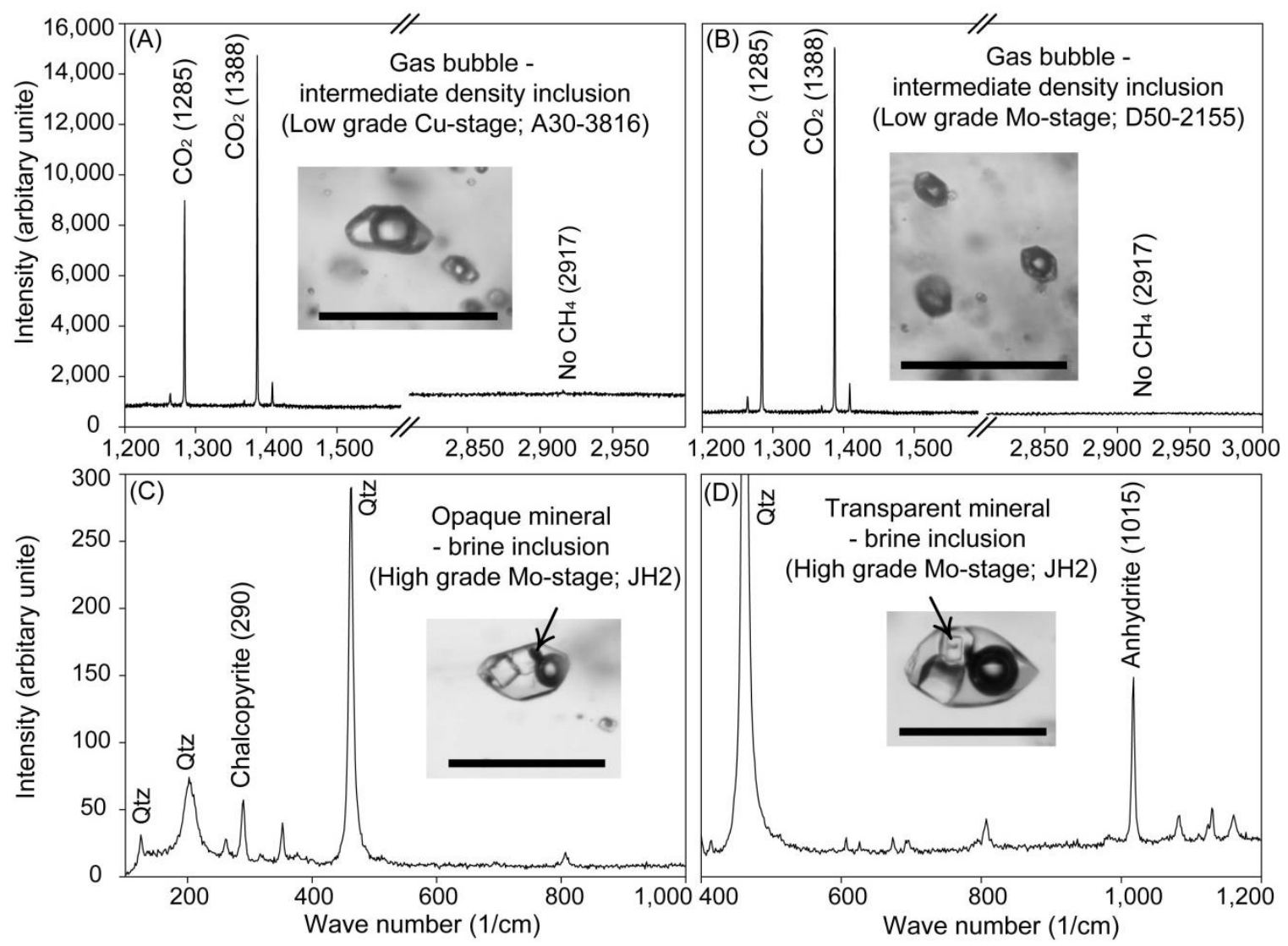

Figure 6. Representative Raman spectrum obtained from bubbles of intermediate-density fluid inclusions from the $\mathrm{Cu}$-stage quartz stockwork (A; A30-3816 sample) and Mo-stage quartz-molybdenite veins (B; D50-2155 sample). These were investigated to check gas species in the intermediate-density fluid inclusions and opaque chalcopyrite and transparent anhydrite daughter crystals in brine inclusions of high Mo grade quartzmolybdenite veins (JH2 sample). The fluid inclusions contain $\mathrm{CO}_{2}$ but do not contain measurable amounts of $\mathrm{CH}_{4}$. Calculated detection limits of $\mathrm{CH}_{4} / \mathrm{CO}_{2}$ molar ratios were near 0.002 corresponding to a $\log f \mathrm{O}_{2}$ near $\mathrm{NNO}+0.3$, suggesting that $\mathrm{CH}_{4}$ detection is difficult in oxidized fluids of $\log f \mathrm{O}_{2}$ higher than $\mathrm{NNO}+0.3$. The precipitated sulfide (chalcopyrite) and sulfate (anhydrite) daughter crystals do not allow accurate determination of oxidized/reduced sulfur species ratios in the fluids. Scale bars in inclusion images are $100 \mu \mathrm{m}$.

\section{Fluid compositional variations}

Salinities of brines range from $31 \pm 1$ to $42 \pm 1 \mathrm{wt} \% \mathrm{NaCl}$ eq. (average $\pm 10 ; 38 \pm 3 \mathrm{wt} \%$ ) in the deep low $\mathrm{Cu}$ grade quartz stockwork veins, and from $40 \pm 1$ to $54 \pm 2$ wt \% $\mathrm{NaCl}$ eq. (average; $48 \pm 4$ wt \%) in the high $\mathrm{Cu}$ grade quartz stockwork veins of the $\mathrm{Cu}-\mathrm{Au}$ orebody (Fig. 5 and Table 2). The salinity increase in the Cu-stage is consistent with the salinity evolution in the central upflow zone documented by Landtwing et al. (2010). In the latestage stockwork vein (post-QLP; 4990-1470 sample), salinities of two brine assemblages ( $40 \pm 1$ and $44 \pm 1$ wt \% $\mathrm{NaCl}$ eq.) are similar to brine assemblages in the high $\mathrm{Cu}$ grade stockwork veins. Salinities of brines in low Mo grade quartz-molybdenite veins vary from $37 \pm 1$ to $51 \pm 1 \mathrm{wt} \% \mathrm{NaCl}$ eq. (average; $42 \pm 3 \mathrm{wt} \%$ ) and in the high Mo grade quartzmolybdenite veins range from $36 \pm 1$ to $59 \pm 2$ wt \% NaCl eq. (average; $44 \pm 5$ wt \%; Fig. 7 
and Table 3). There is a slight trend of increasing salinity from the deep low-grade veins of the Mo-stage to the rich quartz-molybdenite veins, but overall the salinities of the Mo-stage brines overlap with those of the $\mathrm{Cu}-\mathrm{Au}$-stage (Fig. 5 and 7).
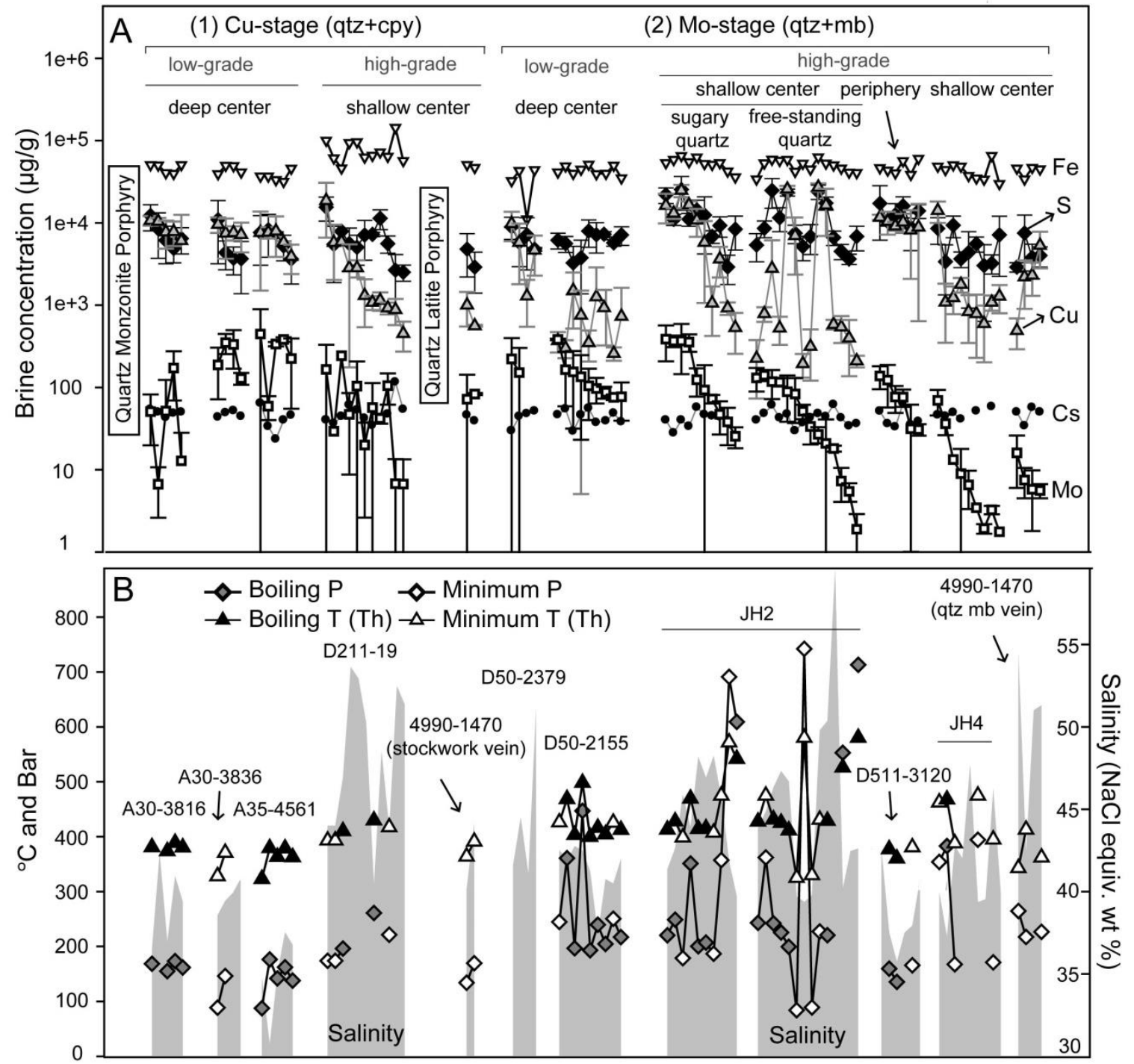

Figure 7. Microthermometry and LA-ICP-MS microanalysis of brine inclusion assemblages from Bingham Canyon. (A) Concentrations of $\mathrm{Fe}, \mathrm{Cu}, \mathrm{Cs}, \mathrm{Mo}$, and $\mathrm{S}$ in the brine inclusion assemblages in low $\mathrm{Cu}$ grade quartz stockwork veins in the barren core (A30-3816, A30-3836, and A35-4561), high Cu grade quartz stockwork vein (D211-19), post-QLP quartz stockwork vein (4990-1470), low Mo grade quartz-molybdenite veins in the barren core (D50-2155 and D50-2379) and high Mo grade quartz-molybdenite veins [JH2 (early sugary quartz and late free-standing quartz; Fig. 3B) D511-3120, JH4, and 4990-1470]. Error bars of S, Cu, and Mo represent one standard deviation in the assemblages. Since these quartz veins contain chalcopyrite (срy) and molybdenite (mb), the $\mathrm{Cu}$-stage (1) and Mo-stage assemblages (2) were sorted by $\mathrm{Cu} / \mathrm{Na}$ and $\mathrm{Mo} / \mathrm{Na}$ ratios, respectively. (B) Measured homogenization temperatures $\left({ }^{\circ} \mathrm{C}\right.$; $\mathrm{T}_{\mathrm{h}}$ ), salinities (wt\% NaCl eqv.), and calculated pressures (bars) of the brine assemblages. Closed symbol represent "boiling assemblage" direct estimations of P-T; Open symbols are from brine-only assemblages representing minimum estimations of P-T.

Consistent copper concentrations within brine inclusion assemblages from the low $\mathrm{Cu}$ grade quartz stockwork veins vary from $4,000 \pm 1,400$ to $11,000 \pm 1,400 \mu \mathrm{g} / \mathrm{g}$ (average; 8,600 \pm $3,200 \mu \mathrm{g} / \mathrm{g}$ ). In each assemblage, they are approximately matched by $\mathrm{S}$ contents ranging between 3,600 $\pm 1,800$ and 12,000 $\pm 4,300 \mu \mathrm{g} / \mathrm{g}$ (average 7,000 $\pm 3,000 \mu \mathrm{g} / \mathrm{g}$; Fig. 7). In the low Mo grade quartz-molybdenite veins, however, $\mathrm{S}$ concentrations from 3,300 $\pm 1,900$ to 
$9,000 \pm 2,700 \mu \mathrm{g} / \mathrm{g}$ (average; 6,000 $\pm 1,500 \mu \mathrm{g} / \mathrm{g}$ ) in brines are variably and sometimes significantly higher than $\mathrm{Cu}$ concentrations that range from $260 \pm 48$ to $9,800 \pm 3,800 \mu \mathrm{g} / \mathrm{g}$ (average $700 \pm 400 \mu \mathrm{g} / \mathrm{g}$ ). Mo concentrations in low-grade veins from both $\mathrm{Cu}$ - and Mostages are comparable, from $7 \pm 4$ to $380 \pm 24 \mu \mathrm{g} / \mathrm{g}$ (average $180 \pm 150 \mu \mathrm{g} / \mathrm{g}$ ) in low $\mathrm{Cu}$ grade quartz stockwork veins and from $80 \pm 6$ to $380 \pm 9 \mu \mathrm{g} / \mathrm{g}$ (average $140 \pm 90 \mu \mathrm{g} / \mathrm{g}$ ) in low Mo grade quartz-molybdenite veins. Fe/Mn atomic ratios in brines from the low $\mathrm{Cu}$ grade stockwork veins between $3.7 \pm 1.2$ and $7.3 \pm 0.4$ are generally lower than those in the low Mo grade quartz-molybdenite veins which vary from $5.3 \pm 0.4$ to $12.0 \pm 2.1$ (Fig. 9 and Table 4).

In high Cu grade quartz stockwork veins (D211-19), Cu concentrations in the brines vary from $450 \pm 180$ to $19,000 \pm 12,000 \mu \mathrm{g} / \mathrm{g}$ and $\mathrm{S}$ concentrations in the brines are from 2,500 \pm 550 to $16,000 \pm 5,000 \mu \mathrm{g} / \mathrm{g}$. The parallel decreases of $\mathrm{Cu}$ with $\mathrm{S}$ are consistent with previously analyzed fluid inclusions in the same sample (D211-19) interpreted to be caused by $\mathrm{Cu}$-sulfide precipitation with falling temperature (Landtwing et al., 2005; Landtwing et al., 2010). Molybdenum concentrations in the high $\mathrm{Cu}$ grade quartz stockwork vein are from $7 \pm 7$ to $360 \mu \mathrm{g} / \mathrm{g}$, in a similar range to low-grade quartz veins from $\mathrm{Cu}$ - and Mo-stages. Two brine inclusion assemblages in late-stage quartz stockwork vein (post-QLP; 4990-1470) contain $560 \pm 14$ and $1,000 \pm 450 \mu \mathrm{g} / \mathrm{g}$ of $\mathrm{Cu}, 2,900 \pm 1,500$ and 4,800 $\pm 2,600 \mu \mathrm{g} / \mathrm{g}$ of $\mathrm{S}$, and $72 \pm 70$ and $83 \pm 2 \mu \mathrm{g} / \mathrm{g}$ of Mo (Table 2 ).

In the high Mo grade quartz-molybdenite vein (JH2 sample; Fig. 7), we observed wide variations of Mo contents (between $2 \pm 1$ and $390 \pm 180 \mu \mathrm{g} / \mathrm{g}$ ) in the brines. In sample JH2, brines in the early sugary quartz with microcrystalline molybdenite contain from $25 \pm 7$ to $390 \pm 180 \mu \mathrm{g} / \mathrm{g}$, and brines in the euhedral quartz that intergrown with flakes of molybdenite contain overlapping, but generally lower Mo from $2 \pm 1$ to $140 \pm 22 \mu \mathrm{g} / \mathrm{g}$ (Fig. 7).

Despite relatively low concentrations of $\mathrm{Cu}$ in all Mo-stage quartz veins (Fig. 7), a few brine assemblages contain extremely enriched $\mathrm{Cu}$ and $\mathrm{S}$ concentrations in the confined $\mathrm{P}-\mathrm{T}$ ranges around $420^{\circ} \mathrm{C}$ and 210 bars (Fig. 7), while maintaining relatively constant $\mathrm{Pb}$ concentrations of 3,000 $\pm 200 \mu \mathrm{g} / \mathrm{g}$. Generally, the concentrations of non-precipitating elements such as $\mathrm{Pb}, \mathrm{Zn}$ and $\mathrm{Cs}$ are constant throughout all fluid stages, especially if normalized to $\mathrm{Na}$ (Table 2 and 3).

Compositional trends of vapor inclusions from the Bingham Canyon deposit are similar to the brines (Fig. 8 and Table 5). Intermediate-density inclusion assemblages in the low $\mathrm{Cu}$ grade quartz stockwork veins have comparable S (S/Na; from 0.22 to 0.88$)$ and $\mathrm{Cu}(\mathrm{Cu} / \mathrm{Na}$; from 0.07 to 0.32 ) contents with some Mo (Mo/Na; from 0.0004 to 0.0054 ). Intermediatedensity inclusion assemblages in the low Mo grade quartz-molybdenite veins contain variable excess of $\mathrm{S}(\mathrm{S} / \mathrm{Na}$; from 0.11 to 0.28$)$ relative to $\mathrm{Cu}(\mathrm{Cu} / \mathrm{Na}$; from 0.01 to 0.11$)$ with some Mo (Mo/Na; from 0.0007 to 0.0018 ; Fig. 8). We observed wide variations of $\mathrm{Mo} / \mathrm{Na}$ ratios (from 0.0001 to 0.0110 ) in the intermediate-density and vapor inclusion ass emblages of high Mo grade quartz-molybdenite veins (A57-6093, JH2, and 4990-1470), similar trends were observed in brines from the same samples.

Cogenetic vapor and brine phase fluids (boiling assemblages) are shown in Figure 11, emphasizing the variable tendency of $\mathrm{Mo}, \mathrm{As}, \mathrm{Au}, \mathrm{Cu}$ and $\mathrm{S}$ enrichment in the vapor inclusions, compared with the salt components Fe, Cs, K and Na. Quartz-hosted vapor + brine inclusion pairs from the $\mathrm{Cu}$-stage and the Mo-stage at Bingham Canyon are compared, as well as data of some Sn-W granites where both S and Mo data are available (Seo et al., 2009; Landtwing et al., 2010). All elements were normalized to the analytically robust $\mathrm{Pb}$ concentration (accurate, precise, and little change of $\mathrm{v} / \mathrm{b}$ partitioning). The data show that Mo selectively fractionates into vapor relative to chloride salts in the $\mathrm{Sn}-\mathrm{W}$-systems and to a lesser but significant degree in the Mo-stage fluids at Bingham Canyon, whereas brine + 
vapor pairs from the $\mathrm{Cu}-\mathrm{Au}$-stage show no significant preferential partitioning or a tendency for Mo to favor the brine phase.

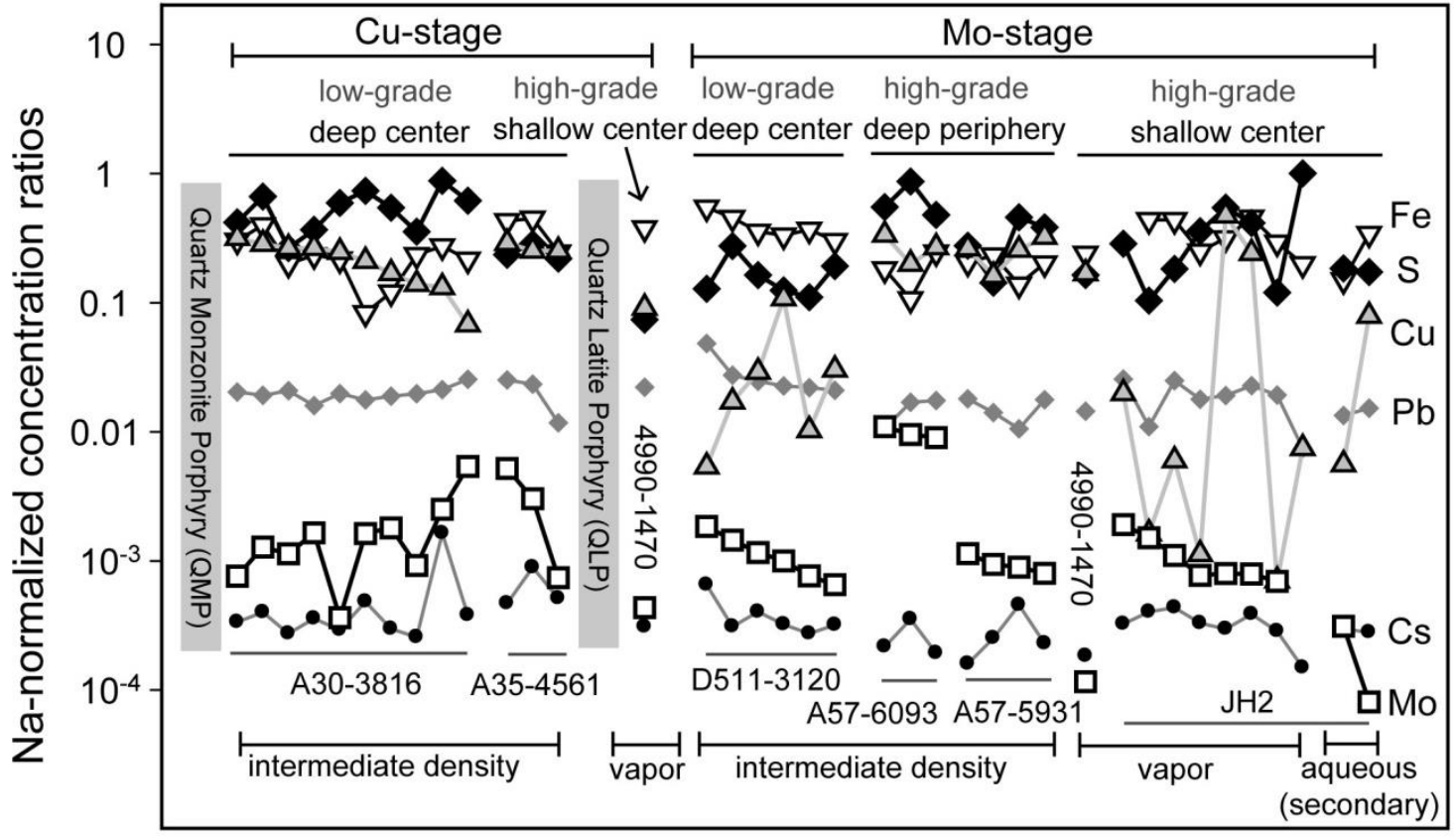

Figure 8. Na-normalized ratios of $\mathrm{Fe}, \mathrm{Cu}, \mathrm{Mo}, \mathrm{Cs}, \mathrm{Pb}$, and $\mathrm{S}$ in the intermediate-density, vapor, and late aqueous inclusion assemblages in low $\mathrm{Cu}$ grade quartz stockwork veins (A30-3816 and A35-4561), post-QLP (Quartz Latite Porphyry) late-stage quartz stockwork vein (4990-1470), low Mo grade quartz-molybdenite vein (D502155), deep peripheral high Mo grade quartz-molybdenite veins (A57-5931, 6093), and shallow high Mo grade quartz-molybdenite veins (JH2 and 4990-1470). We used analytically robust element ratios since it is difficult to obtain accurate element concentrations of these low-salinity fluid inclusions. The assemblages of $\mathrm{Cu}$-stage and Mo-stage are sorted by $\mathrm{Cu} / \mathrm{Na}$ and $\mathrm{Mo} / \mathrm{Na}$ ratios, respectively.

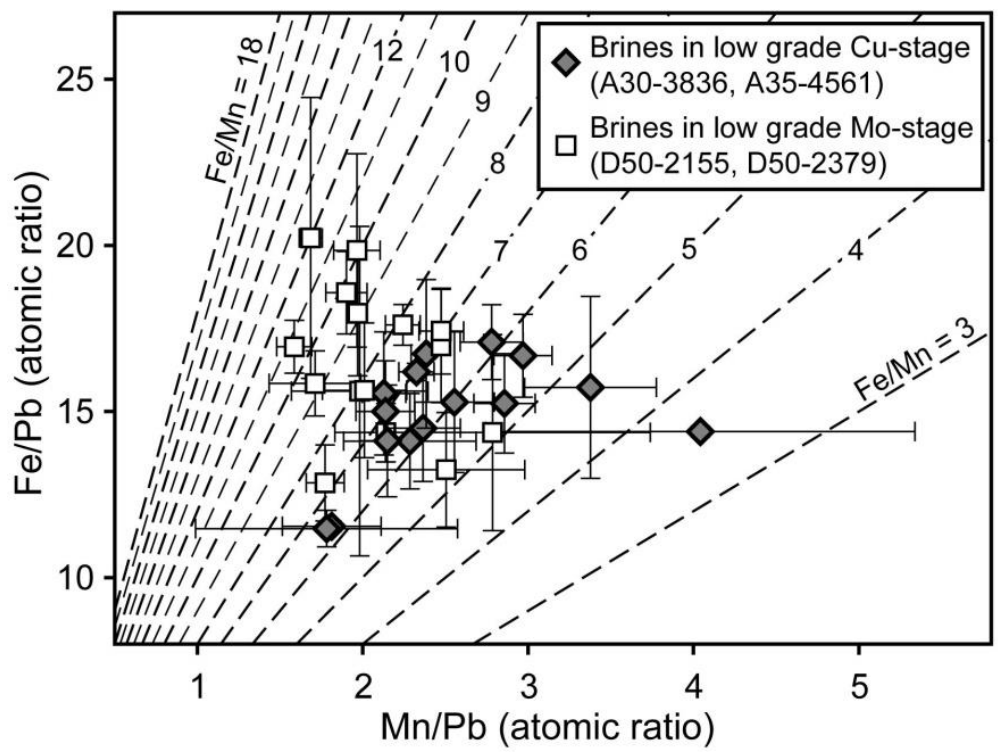

Figure 9. $\mathrm{Fe} / \mathrm{Pb}$ and $\mathrm{Mn} / \mathrm{Pb}$ ppm ratios measured in brine assemblages of low grade Cu quartz stockwork veins (A30-3836, A35-4561) and low grade quartz-molybenite veins (D50-2155, 2379) from the barren core. Contours of $\mathrm{Fe} / \mathrm{Mn}$ ratios from 3 to 18 are presented. $\mathrm{Fe} / \mathrm{Mn}$ ratios of the $\mathrm{Cu}$-stage range from $3.7 \pm 1.2$ to $7.3 \pm$ 0.4 , lower than that of the Mo-stage range, from $5.3 \pm 0.4$ to $12.0 \pm 2.1$. Error bars represent one standard deviation in the assemblages. 
Table 5. Compostion of intermediate density (ID), vapor, and late aqueous fluid inclusion assemblages.

\begin{tabular}{|c|c|c|c|c|c|c|c|c|c|c|c|c|c|c|c|c|c|}
\hline$\mu \mathrm{g} / \mathrm{g}$ & Location & Phase & Sample\# & Sal. & $\operatorname{Th}\left({ }^{\circ} \mathrm{C}\right)$ & $\mathbf{P}($ bar $)$ & ${ }^{23} \mathrm{Na}$ & ${ }^{32} \mathrm{~S}$ & ${ }^{39} \mathrm{~K}$ & ${ }^{57} \mathrm{Fe}$ & ${ }^{65} \mathrm{Cu}$ & ${ }^{75} \mathrm{As}$ & ${ }^{133} \mathrm{Cs}$ & ${ }^{197} \mathrm{Au}$ & ${ }^{208} \mathrm{~Pb}$ & ${ }^{66} \mathrm{Zn}$ & ${ }^{95} \mathrm{Mo}$ \\
\hline Av. & center & ID & A35-4561 & 9.8 & 330 & & 26000 & 6200 & 12000 & 11000 & 7600 & 50 & 12.0 & 0.44 & 660 & ND & 130 \\
\hline SD & deep stw. & & 1_ID1 & ND & & & 6400 & 3900 & 7500 & 5900 & 6100 & 36 & 4.9 & 0.26 & 280 & ND & 210 \\
\hline Av. & center & ID & A35-4561 & 6.2 & & & 16000 & 4700 & 8400 & 7500 & 4200 & 50 & 14.0 & 0.64 & 390 & ND & 50 \\
\hline $\mathrm{SD}$ & deep stw. & & 1_TP2(v) & 1.1 & & & 3600 & 3000 & 4000 & 2900 & 2600 & 57 & 30.0 & 1.10 & 240 & ND & 45 \\
\hline Av. & center & ID & A30-3816 & 8 & 381 & 168 & 23000 & 9800 & 20000 & 7100 & 7400 & 47 & 7.9 & 0.21 & 470 & 490 & 17 \\
\hline SD & deep stw. & & 4_TP11(v) & assum & & & 77 & 1800 & 190 & 1300 & 3300 & 16 & 0.8 & 0.17 & 36 & 49 & 12 \\
\hline Av. & center & ID & A30-3816 & 8 & & & 25000 & 6700 & 14000 & 5100 & 6700 & 42 & 7.0 & 0.44 & 530 & 600 & 29 \\
\hline SD & deep stw. & & 1_ID12 & assum & & & 670 & 2000 & 1700 & 2200 & 2100 & 10 & 1.6 & $\mathrm{ND}$ & 110 & 95 & 23 \\
\hline Av. & center & ID & A30-3816 & 8 & & & 25000 & 15000 & 14000 & 5500 & 6300 & 93 & 7.5 & 3.00 & 500 & 690 & 9 \\
\hline SD & deep stw. & & 1_ID6 & assum & & & 1100 & 5900 & 3200 & 790 & 1500 & 96 & 2.5 & ND & 190 & 200 & 3 \\
\hline Av. & center & ID & A30-3816 & 8 & & & 23000 & 8600 & 20000 & 5500 & 6200 & 56 & 8.4 & ND & 370 & 420 & 39 \\
\hline SD & deep stw. & & 1_ID5 & assum & & & 990 & 3700 & 2700 & 680 & 150 & 25 & 3.5 & ND & 63 & 100 & 27 \\
\hline Av. & center & ID & A30-3816 & 8 & 380 & 162 & 25000 & 18000 & 14000 & 2100 & 5300 & 34 & 12.0 & 0.44 & 450 & 530 & 41 \\
\hline SD & deep stw. & & 4_TP9(v) & assum & & & 970 & 13000 & 2400 & 2000 & 5600 & 15 & 7.8 & 0.45 & 180 & 270 & 25 \\
\hline Av. & center & ID & A30-3816 & 6.2 & 496 & & 17000 & 11000 & 10000 & 7000 & 5000 & 73 & 7.1 & 5.40 & 340 & ND & 22 \\
\hline SD & deep stw. & & 1_ID1 & $\mathrm{ND}$ & & & 1900 & 5000 & 1200 & 1900 & 3100 & 54 & 1.3 & 5.60 & 140 & ND & 8 \\
\hline Av. & center & ID & A30-3816 & 8 & & & 25000 & 13000 & 16000 & 3000 & 4200 & 73 & 7.4 & 0.16 & 470 & 500 & 45 \\
\hline SD & deep stw. & & 1_ID3 & assum & & & 1200 & 6700 & 3400 & 1600 & 1800 & 45 & 2.4 & ND & 170 & 190 & 41 \\
\hline Av. & center & ID & A30-3816 & 8 & & & 24000 & 8700 & 17000 & 5700 & 3400 & 46 & 6.3 & 0.11 & 480 & 460 & 22 \\
\hline SD & deep stw. & & 1_ID4 & assum & & & 1000 & 5500 & 2500 & 3000 & 2800 & 25 & 3.1 & 0.11 & 210 & 190 & 16 \\
\hline Av. & center & ID & A30-3816 & 8 & 374 & 155 & 23000 & 14000 & 19000 & 5100 & 1600 & 38 & 8.9 & 0.24 & 600 & 900 & 120 \\
\hline SD & deep stw. & & 4_TP10(v) & assum & & & 450 & 11000 & 1800 & 6900 & 1800 & 25 & 0.6 & ND & 190 & 590 & 92 \\
\hline Av. & center & ID & A30-3816 & 3.3 & 375 & & 9300 & 8100 & 6800 & 2500 & 1200 & 250 & 15.0 & 8.50 & 190 & ND & 23 \\
\hline SD & deep stw. & & 1_ID2 & $\mathrm{ND}$ & & & 800 & 6300 & 1300 & 1300 & 530 & 400 & 15.0 & 8.40 & 96 & ND & 13 \\
\hline Av. & center & vapor & $4990-1470$ & 8 & ND & ND & 23000 & 1700 & 17000 & 8700 & 2100 & 14 & 7.1 & 0.07 & 510 & 630 & 10 \\
\hline SD & QLP stw. & & 1_V1 & assum & & & 570 & 460 & 1000 & 2200 & 1700 & 9 & 2.1 & 0.04 & 8 & 160 & 10 \\
\hline Av. & center & ID & D50-2155 & 8 & 498 & 447 & 22000 & 2800 & 21000 & 12000 & 120 & ND & 14.0 & ND & 1000 & 1100 & 41 \\
\hline
\end{tabular}




\begin{tabular}{|c|c|c|c|c|c|c|c|c|c|c|c|c|c|c|c|c|c|}
\hline SD & deep moly & & $\mathrm{A} 05(\mathrm{v})$ & assum & & & ND & ND & ND & ND & ND & ND & ND & ND & ND & ND & ND \\
\hline Av. & center & ID & D50-2155 & 8 & 416 & 240 & 24000 & 6600 & 18000 & 10000 & 410 & 21 & 7.4 & 0.18 & 660 & 620 & 35 \\
\hline SD & deep moly & & B03(v) & assum & & & 740 & 4400 & 1800 & 9300 & 850 & 22 & 2.2 & 0.14 & 160 & 150 & 54 \\
\hline Av. & center & ID & D50-2155 & 8 & 399 & 193 & 23000 & 3800 & 20000 & 8200 & 670 & 24 & 9.3 & 0.21 & 570 & 720 & 26 \\
\hline SD & deep moly & & B06(v) & assum & & & 3900 & 1400 & 9900 & 4200 & 680 & 29 & 4.9 & 0.09 & 150 & 590 & 18 \\
\hline Av. & center & ID & D50-2155 & 8 & & & 24000 & 2900 & 18000 & 7900 & 2500 & 11 & 7.7 & 0.48 & 540 & 590 & 24 \\
\hline SD & deep moly & & $\mathrm{A} 01(\mathrm{v})$ & assum & & & 490 & 1300 & 1100 & 790 & 1400 & 4 & 1.0 & 0.46 & 110 & 200 & 8 \\
\hline Av. & center & ID & D50-2155 & 8 & 404 & 205 & 23000 & 2600 & 19000 & 8700 & 240 & 9 & 6.5 & 0.09 & 520 & 640 & 18 \\
\hline SD & deep moly & & B01(v) & assum & & & 570 & 1500 & 1500 & 1500 & 210 & 6 & 3.3 & 0.01 & 95 & 57 & 11 \\
\hline Av. & center & ID & D50-2155 & 8 & 412 & 217 & 24000 & 4600 & 18000 & 7200 & 720 & 10 & 7.7 & 0.11 & 500 & 430 & 15 \\
\hline SD & deep moly & & B02(v) & assum & & & 700 & 3200 & 1700 & 1300 & 920 & 4 & 1.5 & 0.05 & 100 & 140 & 9 \\
\hline Av. & periphery & ID & A57-5931 & 8 & & & 28000 & 13000 & 7400 & 3900 & 7200 & 75 & 12.0 & 1.30 & 290 & 430 & 25 \\
\hline SD & Mo-vein & & ID1 & assum & & & 680 & 6100 & 1600 & 1500 & 1700 & 70 & 9.5 & $\mathrm{ND}$ & 94 & 200 & 25 \\
\hline Av. & periphery & ID & A57-5931 & 8 & & & 27000 & 7600 & 9100 & 5600 & 7200 & 38 & 4.4 & 0.54 & 490 & 550 & 31 \\
\hline SD & Mo-vein & & ID2 & assum & & & 600 & 1000 & 1600 & 1600 & 1600 & 14 & 0.5 & ND & 63 & 91 & 15 \\
\hline Av. & periphery & ID & A57-5931 & 8 & & & 27000 & 10000 & 10000 & 5500 & 8800 & 49 & 6.2 & 0.89 & 480 & 550 & 21 \\
\hline SD & Mo-vein & & ID3 & assum & & & 190 & 2600 & 420 & 1000 & 2200 & 12 & 2.2 & 0.53 & 140 & 110 & 13 \\
\hline Av. & periphery & ID & A57-5931 & 8 & & & 28000 & $\begin{array}{r}4000 \\
1\end{array}$ & 8200 & 6500 & 4500 & 29 & 7.0 & 1.00 & 390 & 480 & 26 \\
\hline SD & Mo-vein & & ID4 & assum & & & 1700 & 100 & 4500 & 2400 & 1300 & 13 & 3.3 & 0.18 & 130 & 200 & 10 \\
\hline Av. & periphery & ID & A57-6093 & 8 & & & 28000 & 15000 & 7300 & 5100 & 9500 & 80 & 6.1 & 3.30 & 300 & 460 & 310 \\
\hline SD & Mo-vein & & ID1 & assum & & & 530 & 4000 & 1500 & 2500 & 2800 & 10 & 2.8 & $\mathrm{ND}$ & 57 & 140 & 400 \\
\hline Av. & periphery & ID & A57-6093 & 8 & & & 27000 & 23000 & 9600 & 2900 & 5400 & 68 & 9.6 & 1.30 & 460 & 660 & 260 \\
\hline SD & Mo-vein & & ID2 & assum & & & 760 & 7000 & 2300 & 1700 & 1500 & 54 & 3.8 & ND & 16 & 220 & 34 \\
\hline Av. & periphery & ID & A57-6093 & 8 & & & 28000 & 13000 & $\begin{array}{r}7300 \\
1\end{array}$ & 6900 & 7500 & 51 & 5.4 & ND & 490 & 610 & 250 \\
\hline SD & Mo-vein & & ID3 & assum & & & 490 & 3000 & 100 & 1400 & 1500 & 28 & 3.1 & ND & 77 & 120 & ND \\
\hline Av. & center & vapor & JH2 & 8 & & & 25000 & 7200 & 14000 & ND & 500 & ND & 8.2 & ND & 650 & 490 & 48 \\
\hline SD & Mo-vein & & 8-1_V3 & assum & & & ND & ND & ND & ND & ND & ND & ND & $\mathrm{ND}$ & ND & ND & ND \\
\hline Av. & center & vapor & $\mathrm{JH} 2$ & 8 & & & 26000 & 2700 & 13000 & 11000 & 42 & 20 & 10.0 & 1.70 & 280 & 660 & 39 \\
\hline SD & Mo-vein & & 8-1_V2 & assum & & & 970 & ND & 2500 & ND & ND & ND & 0.3 & ND & 340 & ND & ND \\
\hline
\end{tabular}




\begin{tabular}{|c|c|c|c|c|c|c|c|c|c|c|c|c|c|c|c|c|c|}
\hline Av. & center & vapor & JH2 & 8 & & & 20000 & 3600 & 18000 & 8800 & 120 & 16 & 8.7 & 0.11 & 500 & 590 & 22 \\
\hline SD & Mo-vein & & 2_V2 & assum & & & 3400 & 2900 & 5400 & 3800 & 93 & 20 & 4.2 & 0.07 & 190 & 310 & 22 \\
\hline Av. & center & vapor & JH2 & 8 & & & 25000 & 8900 & 16000 & 6200 & 28 & 36 & 8.2 & 1.10 & 440 & 320 & 19 \\
\hline SD & Mo-vein & & 3-1_V1 & assum & & & 330 & ND & 900 & 4200 & 22 & 42 & 1.8 & ND & 33 & 3 & 15 \\
\hline Av. & center & vapor & JH2 & 7.9 & 429 & 221 & 22000 & 12000 & 13000 & 7200 & 10000 & 25 & 6.7 & 1.30 & 430 & ND & 18 \\
\hline SD & Mo-vein & & a7(v) & 3.2 & & & 8300 & 10000 & 5600 & 5100 & 10000 & 12 & 4.3 & ND & 240 & ND & 19 \\
\hline Av. & center & vapor & JH2 & 8 & & & 20000 & 8400 & 17000 & 9300 & 4900 & 22 & 7.9 & 0.17 & 460 & 590 & 16 \\
\hline SD & Mo-vein & & 2_V1 & assum & & & 4600 & 7500 & 6600 & 4700 & 8200 & 33 & 5.0 & 0.08 & 180 & 390 & 10 \\
\hline Av. & center & vapor & JH2 & 8 & & & 22000 & 2600 & 15000 & 6500 & 15 & 20 & 6.3 & 0.13 & 430 & 430 & 15 \\
\hline SD & Mo-vein & & 2_V3 & assum & & & 930 & 2000 & 1400 & 1900 & 6 & 18 & 1.0 & 0.10 & 41 & 69 & 8 \\
\hline Av. & center & vapor & JH2 & 8 & & & 26000 & 26000 & 12000 & 5300 & 190 & 79 & 3.9 & 0.90 & 190 & 480 & ND \\
\hline SD & Mo-vein & & 8-1_V1 & assum & & & 610 & 7600 & 1600 & 210 & 49 & 29 & 1.9 & ND & 150 & 98 & ND \\
\hline Av. & center & vapor & JH2 & 7.4 & 492 & 434 & 22000 & 5400 & 11000 & 7000 & 300 & 150 & 9.3 & 3.70 & 540 & ND & 21 \\
\hline SD & Mo-vein & & B_BT2 & 3.1 & & & 3800 & 2500 & 3400 & 1800 & 240 & 29 & 6.9 & 2.90 & 470 & ND & ND \\
\hline Av. & center & vapor & $4990-1470$ & 8 & ND & ND & 25000 & 4100 & 13000 & 6000 & 4200 & 38 & 4.6 & ND & 360 & 430 & 3 \\
\hline SD & Mo-vein & & 2_V1 & assum & & & 850 & 2900 & 1800 & 3300 & 4300 & ND & 0.4 & ND & 55 & 45 & ND \\
\hline Av. & center & aqueous & JH2 & 9.7 & 313 & 97 & 30000 & 5600 & 15000 & 4500 & 170 & 69 & 9.1 & 6.00 & 410 & 690 & 10 \\
\hline SD & Mo-vein & & a8-2_AQ & ND & & & 1700 & 2600 & 2500 & 4500 & ND & 44 & 2.4 & 6.80 & 170 & 360 & 3 \\
\hline Av. & center & aqueous & JH2 & 8 & & & 25000 & 4300 & 15000 & 8600 & 1900 & ND & 7.0 & ND & 380 & 450 & 2 \\
\hline SD & Mo-vein & & 3-1_AQ1 & assum & & & ND & ND & ND & ND & ND & ND & ND & ND & ND & ND & ND \\
\hline
\end{tabular}

Due to difficulties to get reliable salinities from such low-density inclusions, most of the intermediate density (ID) and vapor phase salinities were assumed as 8 equiv. $\mathrm{NaCl}$ wt \% based on previous estimations (Redmond et al., 2004; Landtwing et al., 2010). Only element ratios are analytically-robust and are therefore reported in the current text. Temperatures, pressure data for some vapor inclusions were obtained from brine inclusion data in boiling assemb lages. 

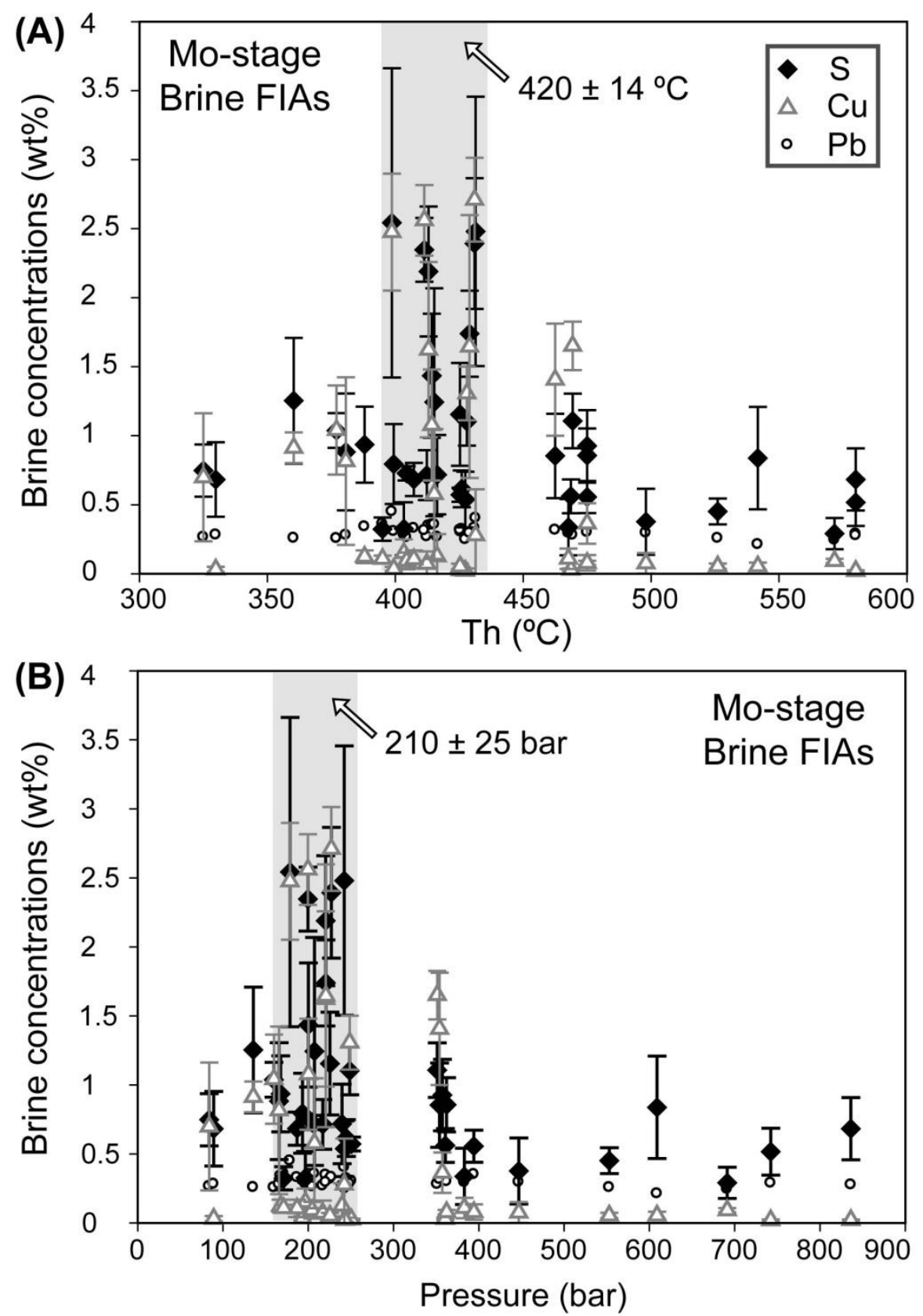

Figure 10. $\mathrm{Cu}, \mathrm{Pb}$, and $\mathrm{S}$ concentrations vs. homogenization temperatures $(\mathrm{A})$ and estimated pressures $(\mathrm{B})$ in the brine fluid inclusion assemblages (FIAs) in the quartz-molybdenite veins, with error bars of $\mathrm{Cu}$ and $\mathrm{S}$ concentrations (1 standard deviation in each assemblage). Anomalously high concentrations of $\mathrm{Cu}$ and $\mathrm{S}$ are confined to ranges of temperature $\left(400-430^{\circ} \mathrm{C}\right.$; $\left.\mathbf{A}\right)$ and pressure $(150-250$ bars; B) where retrograde quartz solubility favors access of the Mo-stage fluids to re-dissolve $\mathrm{Cu}-\mathrm{Fe}$-sulfide grains that were precipitated in the preceding $\mathrm{Cu}$-Au stage.

\section{Discussion}

The presence of intermediate-density inclusions in the deep, low grade quartz-molybdenite veins, and brine and vapor inclusions in the shallow high grade quartz-molybdenite veins, are interpreted to indicate phase separation of a single-phase fluid ascending from depth. From the combined studies of quartz vein textures, fluid inclusion microthermometry, and LA-ICP-MS results, we found only minor differences between the distinct $\mathrm{Cu}$ - and Mo-stages 
with regard to the metal and sulfur contents of the fluids, the prevailing P-T conditions of mineralization, and the evolution of fluids from single-phase intermediate-density to highdensity brine and low-density vapor. The differences may imply that the sharply contrasting metal content of the two vein stages reflects selective metal deposition rather than major differences in fluid characteristics. We argue in the followings that metal separation may be explained by a rather subtle change towards more reducing and possibly acidic conditions in the magmatic fluid source. In addition, local redissolution of earlier ore minerals by later fluids and an unknown degree of post-entrapment diffusional reequilibration may have added to the compositional complexity of the fluid inclusion assemblages.

\section{Magmatic metal fractionation and source magma evolution}

Molybdenum is an incompatible element in a crystallizing magma (Candela and Holland, 1984; Keppler and Wyllie, 1991), so that fractional crystallization accumulates Mo in the residual melt (Audétat, 2010). As a result later stage magma is expected to exsolve the most Mo-rich fluids. Fluid-conservative elements including $\mathrm{Cs}, \mathrm{Pb}$, and $\mathrm{Zn}$ are also incompatible during magmatic fractionation (Audétat and Pettke, 2003) but do not precipitate as distinct mineral phases in the core of porphyry deposits. Concentrations of these elements in the fluids do not change significantly from the $\mathrm{Cu}$ - to the Mo-stage at Bingham Canyon, and $\mathrm{Pb}$ isotope ratios in the fluid inclusions show that the fluids responsible for the $\mathrm{Cu}$ and Mo stage ores in the Bingham Canyon deposit originated from the same magmatic source (Pettke et al., 2010). These observations argue against fundamental differences in source magmas or a highly contrasting degree of magma fractionation, as a cause for the distinct metal endowments of the two mineralization stages at Bingham Canyon. Indeed, the Mostage fluids are not more Mo-rich, but they tend to be depleted in $\mathrm{Cu}$, which is also incompatible in the magma but might be removed by early-exsolving, S-rich magmatic vapor phase (Zajacz and Halter, 2009). Copper depletion with continued high Mo supply could therefore reflect a process of metal segregation during progressive fluid separation from a single, progressively crystallizing magma reservoir.

\section{$P$-T conditions and extent of phase separation}

The temperature and pressure of late Mo stage fluids of a maximum of $580{ }^{\circ} \mathrm{C}$ with an estimated pressure of up to 710 bar (Fig. 5) overlap with the maximum pressure around $500{ }^{\circ} \mathrm{C}$ and 800 bars for $\mathrm{Cu}-\mathrm{Au}$ stage fluids from barren core estimated by Landtwing et al. (2010), suggesting that the P-T conditions of the deep input intermediate-density phase fluids of the two ore-forming stages ( $\mathrm{Cu}$ and Mo) were essentially constant within our observational uncertainty. The salinity ranges of brines in both ore stages overlap as well (Fig. 5), indicating that single phase fluids in early $\mathrm{Cu}$ - to late Mo-stages decompressed and cooled, and underwent similar extents of brine + vapor phase separation. Following the mass-balance approach of Landtwing et al. (2010) using fluid salinity and analyzed element ratio with the 'lever rule' applied to $\mathrm{H}_{2} \mathrm{O}-\mathrm{NaCl}$ model system (Driesner and Heinrich, 2007), we calculated the mass fractions of the brine and vapor in the system responsible for the Mo-stage by using the observed salinities and element ratios. The element/Na ratios of intermediate-density fluid inclusion assemblages in low-grade sample (D50-2155) and two boiling assemblages in shallow high-grade samples (JH2_a7 and BT2; Seo et al., 2009) were taken as representative to estimate the mass fractions of separating vapor and brine in the Mo-stage fluid plume (Table 6). For the calculation, we assumed the initial salinity of the intermediate-density fluids as $8 \mathrm{wt} \% \mathrm{NaCl}$ equiv. based on previous estimates (Redmond et al., 2004; Landtwing et al., 2010). The element ratios in the intermediate-density fluids, and the salinities and element ratios in the brines in the boiling assemblages are adopted to predict the salinity and element ratios of the coexisting vapor inclusions in the boiling 
Table 6. Mass-balance calculation to estimate vapor composition and vapor-brine mass fractions.

\begin{tabular}{|c|c|c|c|c|c|c|c|c|c|c|c|c|}
\hline & & \multicolumn{2}{|c|}{$\begin{array}{c}\text { Intermediate-density } \\
\text { Analyzed (average all ID) }\end{array}$} & \multicolumn{3}{|c|}{$\begin{array}{l}\text { Brine in boiling assemblage } \\
\text { Analyzed (JH2_BT2) }\end{array}$} & \multicolumn{3}{|c|}{$\begin{array}{c}\text { Calculated vapor } \\
\left(100 \mathrm{~g} \text { ID fluid }-\mathrm{X}^{*} 100 \mathrm{~g} \text { Brine }\right)\end{array}$} & \multicolumn{3}{|c|}{$\begin{array}{l}\text { Vapor in boiling assemblage } \\
\text { Analysed (JH2_BT2) }\end{array}$} \\
\hline & & \multirow[b]{4}{*}{$\mathrm{Na}$ wt $\%=$} & \multirow{3}{*}{$\begin{array}{c}\text { Salinity } \\
\text { wt } \% \mathrm{NaCl} \text { eq. } \\
\underline{\mathbf{8 . 0}}\end{array}$} & \multirow{3}{*}{\multicolumn{2}{|c|}{$\begin{array}{c}\text { Salinity } \\
\text { wt \% } \mathrm{NaCl} \text { eq. } \\
\mathbf{4 1 . 7}\end{array}$}} & \multirow{4}{*}{$\begin{array}{c}\text { Fluid } \\
\text { Mass } \\
\text { Fraction: } \\
\mathbf{0 . 1}\end{array}$} & \multirow[b]{4}{*}{$\mathrm{Na} w \mathrm{t} \%=$} & \multirow{4}{*}{$\begin{array}{c}\text { Salinity } \\
\text { wt \% } \mathrm{NaCl} \text { eq. } \\
4.5 \\
1.1\end{array}$} & \multirow{4}{*}{$\begin{array}{c}\text { Fluid } \\
\text { Mass } \\
\text { Fraction: } \\
0.9\end{array}$} & \multirow{4}{*}{$\begin{array}{c}\text { Salinity } \\
\text { wt \% } \mathrm{NaCl} \\
\text { eq. } \\
\mathbf{7 . 4}\end{array}$} & \multirow{2}{*}{\multicolumn{2}{|c|}{ Error $(1 \sigma)$}} \\
\hline & & & & & & & & & & & & \\
\hline & & & & & & & & & & & 3.1 & 3.1 \\
\hline & & & 2.1 & $\mathrm{Na} w \mathrm{t} \%=$ & 11.6 & & & & & & & \\
\hline Com- & $m M C l / m M$ & Ratios & Conc. wt \% & Ratios & Conc. wt\% & Comp. & Ratios & Conc. wt $\%$ & Comp. & El-Ratio & Error & $(1 \sigma)$ \\
\hline $\begin{array}{l}\text { ponent } \\
\text { (a) }\end{array}$ & (b) & $\begin{array}{c}\mathrm{M} / \mathrm{Na} \\
(\mathrm{ICP}) \\
(\mathrm{c})\end{array}$ & $\begin{array}{c}(\mathrm{gMCl} / 100 \mathrm{~g} \\
\text { ID) } \\
\text { (d) }\end{array}$ & $\begin{array}{c}\mathrm{M} / \mathrm{Na} \\
(\mathrm{ICP}) \\
(\mathrm{e})\end{array}$ & $\begin{array}{l}\text { (gMCl } / 100 \mathrm{~g} \\
\text { B) } \\
\text { (f) }\end{array}$ & $\begin{array}{l}\text { Fraction } \\
\qquad(\mathrm{g})\end{array}$ & $\begin{array}{c}\mathrm{M} / \mathrm{Na} \\
\text { (pred) } \\
(\mathrm{h})\end{array}$ & $\begin{array}{l}(\mathrm{gMClx} / 100 \mathrm{gV}) \\
\text { (i) }\end{array}$ & $\begin{array}{l}\text { Fraction } \\
\qquad(\mathrm{k})\end{array}$ & $\begin{array}{c}\mathrm{M} / \mathrm{Na}(\mathrm{ICP}) \\
\text { (l) }\end{array}$ & $\begin{array}{c}+ \\
(\mathrm{m})\end{array}$ & (n) \\
\hline $\mathrm{NaCl}$ & 2.54 & 1 & 5.345 & 1 & 29.428 & 2.943 & 1 & 2.670 & 2.403 & 1 & $\mathbf{0}$ & $\mathbf{0}$ \\
\hline $\mathrm{KCl}$ & 1.91 & 0.840 & 3.368 & 0.684 & 15.089 & 1.509 & 1.032 & 2.065 & 1.859 & 0.522 & 0.158 & 0.158 \\
\hline $\mathrm{FeCl}_{2}$ & 2.27 & 0.390 & 1.861 & 0.340 & 8.924 & 0.892 & 0.452 & 1.077 & 0.969 & 0.324 & 0.084 & 0.084 \\
\hline $\mathrm{S}$ & 1 & 0.170 & 0.357 & 0.053 & 0.609 & 0.061 & 0.314 & 0.329 & 0.297 & 0.266 & 0.164 & 0.164 \\
\hline $\mathrm{Cu}$ & 1 & 0.033 & 0.069 & 0.011 & 0.122 & 0.012 & 0.061 & 0.064 & 0.057 & 0.016 & 0.017 & 0.016 \\
\hline $\mathrm{PbCl}_{2}$ & 1.34 & 0.028 & 0.079 & 0.034 & 0.526 & 0.053 & 0.021 & 0.029 & 0.026 & 0.026 & 0.023 & 0.023 \\
\hline Mo & 1 & 1.10E-03 & $2.31 \mathrm{E}-03$ & 7.95E-04 & $9.20 \mathrm{E}-03$ & $9.20 \mathrm{E}-04$ & $1.47 \mathrm{E}-03$ & $1.55 \mathrm{E}-03$ & $1.39 \mathrm{E}-03$ & 8.65E-04 & & \\
\hline $\mathrm{Au}$ & 1 & 1.00E-05 & $2.10 \mathrm{E}-05$ & 1.14E-05 & $1.32 \mathrm{E}-04$ & $1.32 \mathrm{E}-05$ & $8.27 \mathrm{E}-06$ & $8.68 \mathrm{E}-06$ & 7.81E-06 & $1.80 \mathrm{E}-04$ & 0.0001 & 0.0001 \\
\hline As & 1 & 0.0005 & 0.0011 & 0.0004 & 0.0050 & 0.0005 & 0.0007 & 0.0007 & 0.0006 & 0.0068 & 0.0014 & 0.0014 \\
\hline \multirow[t]{2}{*}{$\mathrm{CsCl}$} & 1.27 & 0.0004 & 0.0010 & 0.0004 & 0.0055 & 0.0005 & 0.0004 & 0.0005 & 0.0005 & 0.0004 & 0.0003 & 0.0003 \\
\hline & & TDS wt \% & 11.08 & TDS wt \% & 54.7 & 5.47 & TDS wt \% & 6.24 & 5.61 & & & \\
\hline
\end{tabular}

Modeling procedure and assumptions following Landtwing et al. (2010, Table 2). Concentrations of intermediate density (ID) fluid inclusions in deep low Mo grade quartzmolybdenite vein (D50-2155) and concentrations of brine inclusions in boiling assemblage (BT2) in shallow high Mo grade quartz-molybdenite vein (JH2) were measured by LA-ICP-MS. Input fluid is based on average element ratios of all intermediate density fluid inclusions (column c). Element ratios and salinities of brine inclusions in boiling assemblage (column e) were adopted, whereas the poorly known salinities of intermediate density fluids, vapor and the mass fraction brine and vapor were varied to predict element ratios and salinity of vap or inclusions. The predicted element ratios were compared with analyzed element ratios (column I) to obtain an approximation satisfying all analytical constraints within their estimated uncertainty (Fig. 12). $\mathrm{mMCl}, \mathrm{mM}$, and $\mathrm{gMCl}$ molar mass of salt components, molar mass of elements, and weight (g) of salt components, respectively. Comp., pred, Conc., El-Ratio, and TDS represent composition, predicted value, concentration, element ratio, and total dissolved solids, respectively. 
assemblages. By varying the fluid mass fractions of brine and salinity of the intermediatedensity fluids, we predicted vapor salinities and element concentrations that match with the actually analyzed element ratios in the vapor inclusions, without generating negative concentration values (Table 6 and Fig. 12; c.f. Landtwing et al., 2010, Table 2 for procedure and assumptions). Results indicate that the deep single-phase fluid of the Mo stage separated into approximately $90 \%$ vapor and $10 \%$ brine by mass, although even higher vapor predominance (up to $\sim 99 \%$ ) is permitted by the compositional constraints. The fluid mass ratio of vapor/brine $~ 9: 1$ implies, based on the analyzed metal concentrations in the two boiling pairs (Seo et al., 2009; Fig. 11), that at least $70 \%$ of the Mo in the deposit was precipitated from the vapor phase.

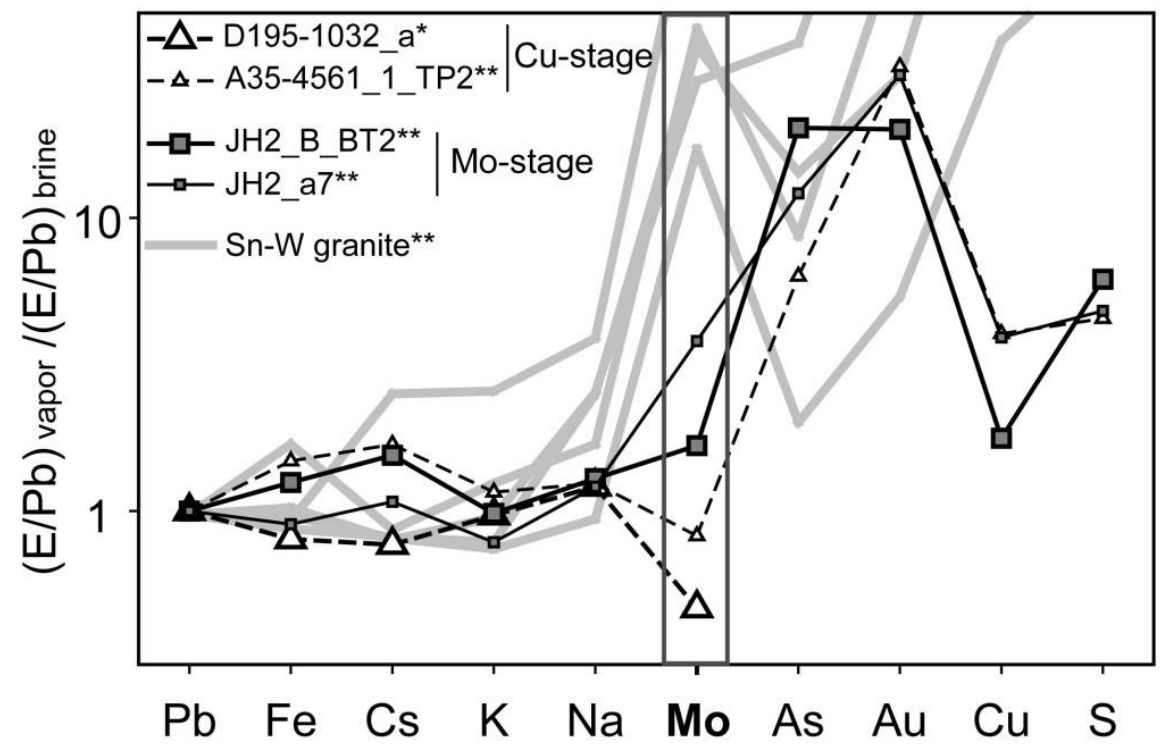

Figure 11. Elements ratios between coeval vapor and brine inclusions in cogenetic assemblages (boiling assemblage) in $\mathrm{Cu}-\mathrm{Au}$ (D195-1032_a and A35-4561_1_TP2) and Mo-stages (JH2_B_BT2 and JH2_a7) in the Bingham Canyon deposit and the Sn-W-mineralized Mole Granite, including data from Landtwing et al. (2010) and Seo etal. (2009). Elements were normalized to $\mathrm{Pb}$ concentration (as an analytically more robust proxy for $\mathrm{Cl}$ and other chloride-complexed metals including $\mathrm{Fe}, \mathrm{Cs}, \mathrm{Na}$; also $\mathrm{K}, \mathrm{Rb}, \mathrm{Mn}, \mathrm{Zn}, \mathrm{Sr}, \mathrm{Ba}$ etc.), to show re lative enrichment of $\mathrm{S}, \mathrm{As}, \mathrm{Au} \pm \mathrm{Mo} \pm \mathrm{Cu}$ in the vapor inclusions. Molybdenum is particularly enriched in vapor in the Sn-W granites (possibly due to more reduced and acidic fluid conditions) compared to the Bingham Canyon porphyry deposits. Among the fluid inclusion assemblages at Bingham Canyon, Mo partitioned more into the vapor during the Mo-stage than during the $\mathrm{Cu}$-stage.

The greater concentration of the Mo ore shell towards the barren core (Fig. 1) and our microthermometric data indicate that fluids ascending during the Mo-stage started saturating molybdenite at somewhat higher pressure and temperature than the apparently quite restricted temperature window of $\mathrm{Cu}-\mathrm{Au}$ deposition below $420^{\circ} \mathrm{C}$ (Landtwing et al., 2005). The fluid phase evolution (from intermediate-density to brine+vapor) as indicated by salinity constraints, width of solvus (Fig. 5), and mass balance of 9/1 ratios of vapor/brine remained essentially identical through both mineralization stages. These observations indicate that the P-T structure of the fluid plume was relatively stationary throughout the evolution from $\mathrm{Cu}$-Au-stage to Mo-stage mineralization. This is consistent with recent geochronological evidence that all $\mathrm{Cu}-\mathrm{Au}$ mineralization pulses occurred within a short but unresolved time interval of U-Pb zircon ages between 38.10 and $37.78 \mathrm{Ma}$ (von Quadt et al., 2011), but is difficult to reconcile with Re-Os data suggesting that the Mo mineralization followed half a million years later at $37.00 \pm 0.27$ (Chesley and Ruiz, 1997). 


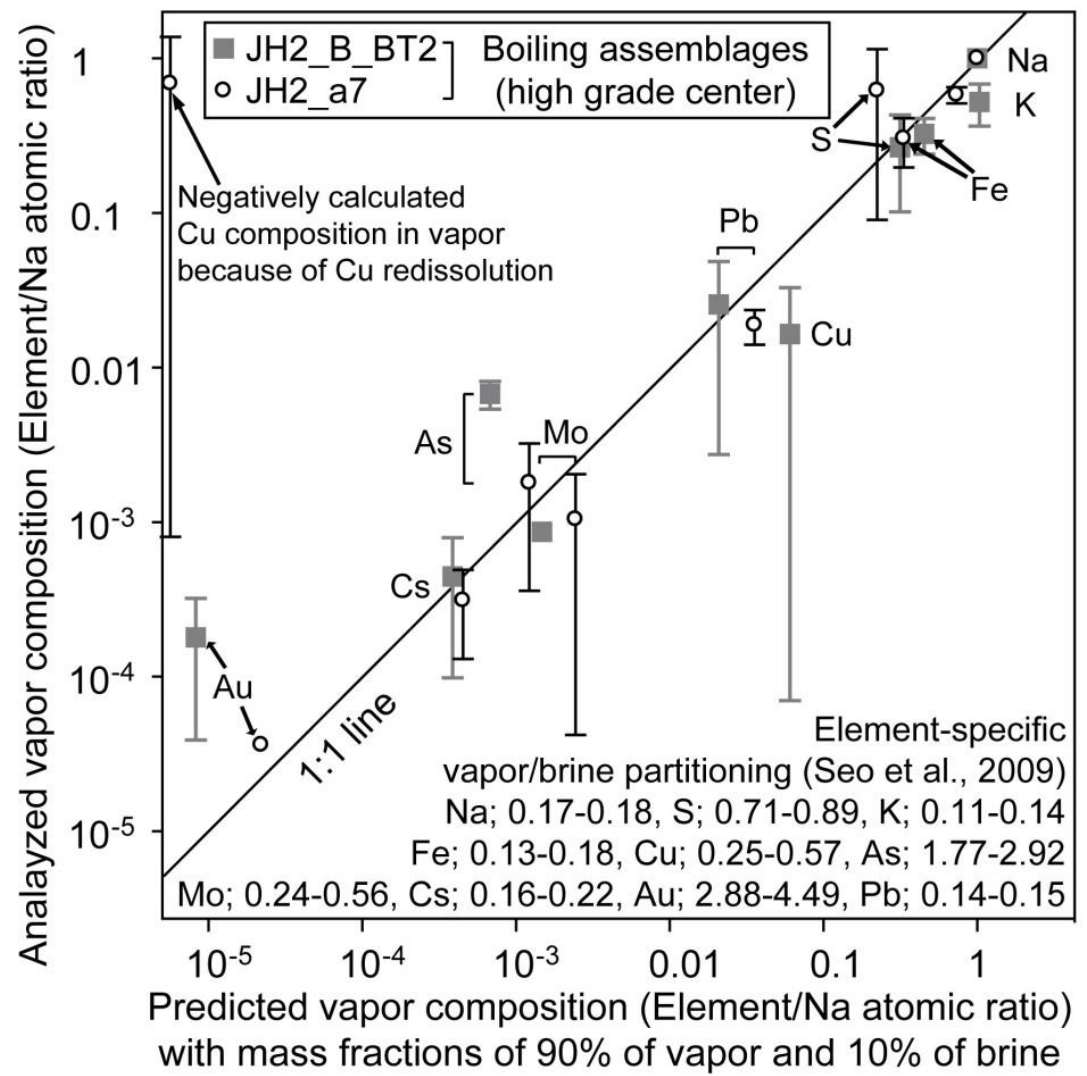

Figure 12. Comparison of best-estimate (mass balance prediction) vs. analyzed (LA-ICP-MS) vapor composition ratios during the Mo-stage. Mass proportions of $90 \%$ vapor and $\sim 10 \%$ brine are estimated based on the composition of intermediate-density fluid inclusions (low-grade Mo-stage sample D50-2155) and two boiling assemblages from a high grade quartz-molybdenite vein (B_BT2 and a7 in JH2). The calculation procedure is provided in the text and Table 6, following the approach of Landtwing et al. (2010) and including data from Seo et al. (2009).

\section{Redox and $p H$ affecting ore-metal precipitation}

The precipitation of chalcopyrite, bornite, and molybdenite as major ore minerals in porphyry-style deposits depends on sulfide activity and requires an adequate quantity of sulfur in the hydrothermal fluids. Since the intermediate-density, brine, and vapor fluids contain enough $\mathrm{S}$ to precipitate all of their $\mathrm{Cu}$ and $\mathrm{Mo}$ as $\mathrm{CuFeS}_{2}$ and $\mathrm{MoS}_{2}$, the dominant ore minerals at Bingham Canyon (Figs. 7 and 8), a relatively small temporal change in redox conditions and acid balance within the magmatic fluid source may be responsible for largescale metal separation by selective sulfide precipitation.

The stoichiometry of $\mathrm{Cu}$ and Mo sulfide precipitation reactions shows that a reduction of redox potential (lower $\mathrm{fO}_{2}$ ) and an increase in the acidity (lower $\mathrm{pH}$ ) in the hydrothermal fluids entering the Bingham Canyon deposit have opposing effects on the saturation of chalcopyrite and molybdenite, and may therefore trigger a switch from the first stage of predominant $\mathrm{Cu}$-Fe-sulfide $\pm \mathrm{Au}$ deposition to a second stage of preferential precipitation of molybdenite.

Chemical reactions for chalcopyrite precipitation from vapor-like fluids are likely to involve $\mathrm{Cu}(\mathrm{I})-\mathrm{S}$ complexes such as $\mathrm{Cu}(\mathrm{HS})_{2}-$ and $\mathrm{Cu}(\mathrm{HS})\left(\mathrm{H}_{2} \mathrm{~S}\right)$ (Pokrovski et al., 2008; Seo et al., 2009; Etschmann et al., 2010):

$$
\begin{aligned}
& 2 \mathrm{Cu}\left(\mathrm{HS}_{2}\right)^{-}+2 \mathrm{FeCl}_{2 \text { (aq) }} \rightarrow 2 \mathrm{CuFeS}_{2}+2 \mathrm{Cl}+2 \mathrm{HCl}_{\text {(aq) }}+\mathrm{H}_{2 \text { (aq) }} \\
& 2 \mathrm{Cu}(\mathrm{HS})\left(\mathrm{H}_{2} \mathrm{~S}\right)_{\text {(aq) }}+2 \mathrm{FeCl}_{2 \text { (aq) }} \rightarrow 2 \mathrm{CuFeS}_{2}+4 \mathrm{HCl}_{\text {(aq) }}+\mathrm{H}_{2 \text { (aq) }}
\end{aligned}
$$


whereas deposition from the volumetrically minor brine phase probably includes chloridecomplexed Cu(I) (Crerar and Barnes, 1976; Mountain and Seward, 1999, 2003).

$$
\mathrm{CuCl}_{(\mathrm{aq})}+\mathrm{FeCl}_{2(\mathrm{aq})}+2 \mathrm{H}_{2} \mathrm{~S}_{(\mathrm{aq})} \rightarrow \mathrm{CuFeS}_{2}+3 \mathrm{HCl}_{(\mathrm{aq})}+0.5 \mathrm{H}_{2(\mathrm{aq})}
$$

All these reactions show that chalcopyrite saturation is promoted by oxidized fluid conditions (low $\mathrm{H}_{2}$ ) and low acidity (low $\mathrm{HCl}$ concentration, i.e. more neutral $\mathrm{pH}$ ).

Possible reactions for $\mathrm{Mo}(\mathrm{IV}) \mathrm{S}_{2}$ precipitation can be formulated by using previously suggested Mo(VI) complexes in high-temperature hydrothermal fluids (Candela and Holland, 1984; Rempel et al., 2008; Ulrich and Mavrogenes, 2008; Rempel et al., 2009; Minubayeva and Seward, 2010).

$$
\begin{aligned}
& \mathrm{MoO}_{3 \text { (aq) }}+\mathrm{H}_{2(\mathrm{aq})}+2 \mathrm{H}_{2} \mathrm{~S}_{(\mathrm{aq})} \rightarrow \mathrm{MoS}_{2}+3 \mathrm{H}_{2} \mathrm{O} \\
& \mathrm{MoO}_{4}^{2-}+2 \mathrm{H}^{+}+\mathrm{H}_{2 \text { (aq) }}+2 \mathrm{H}_{2} \mathrm{~S}_{\text {(aq) }} \rightarrow \mathrm{MoS}_{2}+4 \mathrm{H}_{2} \mathrm{O} \\
& \mathrm{HMoO}_{4}^{-}+\mathrm{H}^{+}+\mathrm{H}_{2 \text { (aq) }}+2 \mathrm{H}_{2} \mathrm{~S}_{\text {(aq) }} \rightarrow \mathrm{MoS}_{2}+4 \mathrm{H}_{2} \mathrm{O} \\
& \mathrm{H}_{2} \mathrm{MoO}_{4(\text { (aq) }}+\mathrm{H}_{2 \text { (aq) }}+2 \mathrm{H}_{2} \mathrm{~S}_{\text {(aq) }} \rightarrow \mathrm{MoS}_{2}+4 \mathrm{H}_{2} \mathrm{O} \\
& \mathrm{MoO}_{2} \mathrm{Cl}^{+}+\mathrm{H}_{2 \text { (aq) }}+\mathrm{HS}^{-}+\mathrm{H}_{2} \mathrm{~S}_{\text {(aq) }} \rightarrow \mathrm{MoS}_{2}+2 \mathrm{H}_{2} \mathrm{O}+\mathrm{HCl}_{\text {(aq) }} \\
& \mathrm{MoO}_{2} \mathrm{Cl}_{2 \text { (aq) }}+\mathrm{H}_{2(\mathrm{aq})}+2 \mathrm{H}_{2} \mathrm{~S}_{(\mathrm{aq})} \rightarrow \mathrm{MoS}_{2}+2 \mathrm{H}_{2} \mathrm{O}+2 \mathrm{HCl}_{(\mathrm{aq})} \\
& \mathrm{MoO}_{2} \mathrm{Cl}_{3^{-}}+\mathrm{H}_{2 \text { (aq) }}+2 \mathrm{H}_{2} \mathrm{~S}_{\text {(aq) }} \rightarrow \mathrm{MoS}_{2}+2 \mathrm{H}_{2} \mathrm{O}+2 \mathrm{HCl}_{\text {(aq) }}+\mathrm{Cl}^{-}
\end{aligned}
$$

To precipitate $\mathrm{MoS}_{2}$ from oxy, hydroxy or oxy-chloride complexes of Mo(VI) will always require $\mathrm{H}_{2} \mathrm{~S}$ and $\mathrm{H}_{2}$ (reactions 4-10). The solubility equilibria thus indicate that high $\mathrm{H}_{2} \mathrm{~S}$ activities and net reduction will favor molybdenite precipitations (Einaudi et al., 2003; Seedorff and Einaudi, 2004b). In the case of oxy- and hydroxy species, the reactions are either $\mathrm{H}^{+}$-independent $(4,7)$ or consume acidity $(5,6)$. These molybdate species are likely to predominate in vapor-dominated systems in which Mo preferentially partitions into the vapor phase (Fig. 11), but a similar redox (and $\mathrm{pH}$ ) dependence also applies if chlorocomplexes were contributing to Mo transport.

Based on these contrasting effects of redox and $\mathrm{pH}$ on $\mathrm{Cu}$ and Mo precipitation reactions, we propose that a small difference in redox potential and acidity level in the mineralizing fluid may tip the balance of metal competition for a limited amount of sulfide in solution. If input fluid conditions during the early $\mathrm{Cu}-\mathrm{Au}$ stage were relatively oxidizing (i.e. $\log \mathrm{fO}_{2}$ of about NNO+1; Audétat and Pettke, 2006) and alkaline, the saturation of $\mathrm{MoS}_{2}$ upon cooling may have been retarded, allowing $\mathrm{Cu}-\mathrm{Fe}$ sulfides to saturate first (reactions 1-3). If $\mathrm{Cu}$ and $\mathrm{S}$ concentrations are similar initially, the precipitation of $\mathrm{Cu}$-Fe sulfide would rapidly deplete the $\mathrm{S}$ concentration in the fluid, preventing molybdenite precipitation even at lower temperature (Fig. 13A). As a consequence, the Mo advected by the fluids during the Cu-stage would be flushed out into the lithocap or into groundwater above the present erosion level (Williams-Jones and Heinrich, 2005; Arnorsson and Oskarsson, 2007), or be precipitated outside the porphyry-style orebody where the fluids met a S-rich reducing environment. Conversely, slightly more reducing conditions for the Mo-stage fluid may allow molybdenite to saturate first upon cooling, leading to effective $\mathrm{MoS}_{2}$ deposition from an $\mathrm{H}_{2} \mathrm{~S}$-rich fluid (reactions 4-10), followed by chalcopyrite saturation at slightly lower temperatures because of the more reducing and acidic conditions (reactions 1-3) and the lower initial $\mathrm{Cu}$ concentrations at similar S level (Fig. 13B).

The combined chemical effects of changes in redox and $\mathrm{pH}$ together with a steep temperature and pressure dependence in metal solubility are consistent with the mine scale distribution and the textural occurrences of chalcopyrite and molybdenite. Maximum Mo ore grades are displaced down and inward relative to the $\mathrm{Cu}$ ore shell (Fig. 1), consistent 
with initial high-temperature saturation of molybdenite during the Mo-stage. Since Mo will never consume all $\mathrm{S}$, and since even the Mo-stage fluid contained $\mathrm{Cu}$ in excess of Mo, it will eventually saturate $\mathrm{Cu}-\mathrm{Fe}$ sulfide at somewhat lower temperature (Fig. 13B). This is consistent with the observation that molybdenite commonly precipitated as the first mineral, directly on the walls of the Mo stage veins, and was followed later by chalcopyrite in the central vug space of quartz-molybdenite veins (Fig. 2). Some of the late $\mathrm{Cu}$ introduced during the Mo-stage may also contribute to the $\mathrm{Cu}$-Fe sulfides that are now hosted in texturally late positions in earlier quartz stockwork veins. This would have led to a cryptic upgrading of the $\mathrm{Cu}$ ore shell surrounding the zone of greatest Mo enrichment.
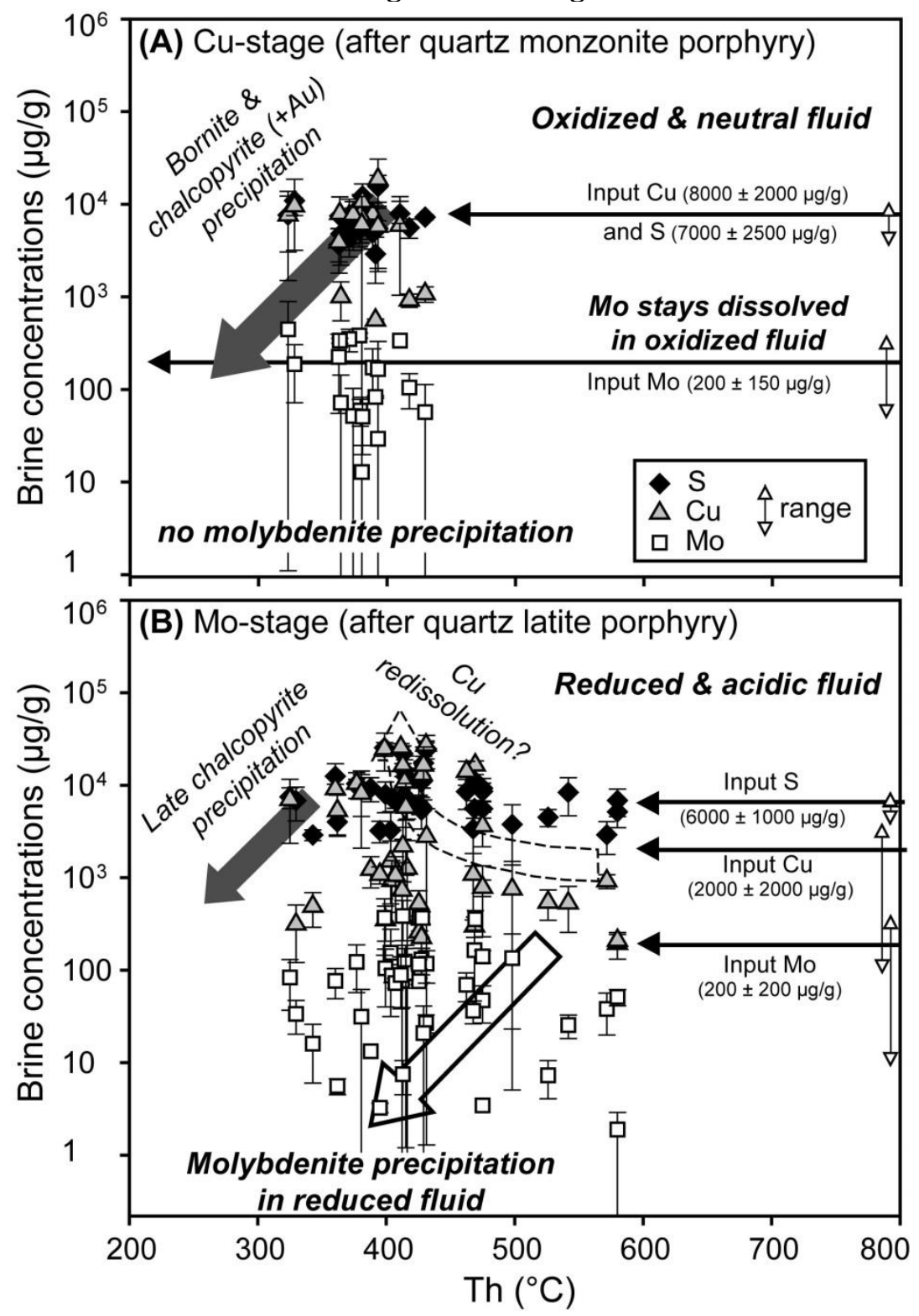

Figure 13. Sulfur, $\mathrm{Cu}$, and Mo concentrations in brine inclusions and interpreted mechanisms responsible for the selective precipitations of $\mathrm{Cu}$-Fe sulfide during the early $\mathrm{Cu}$-stage $(\mathbf{A})$ and molybdenite precipitation during the late Mo-stage (B) During the $\mathrm{Cu}$-stage, relatively oxidized and neutral fluids are inferred to have exclusively precipitated $\mathrm{Cu}-\mathrm{Fe}$ sulfides and $\mathrm{Au}$, whereas Mo stayed dissolved in S-depleted fluids. During the Mo-stage, relatively reduced and acidic fluids favored molybdenite precipitation. Copper sulfide re-dissolution (Fig. 10) might have led to precipitation of late euhedral chalcopyrite in the vein center of quartz-molybdenite veins (see Fig. 2). 
Independent evidence for a change towards more reducing and acid conditions from the early $\mathrm{Cu}-\mathrm{Au}$ to the later Mo stage is weak, in light of the simple and non-diagnostic vein mineralogy, but permissive. Higher fluid acidity in the Mo-stage is indicated by the occurrence of feldspar-destructive alteration haloes, despite high temperature, along quartz-molybdenite veins, especially where a massive molybdenite seam directly overgrows the vein wall (Fig. $2 \mathrm{~B}$ and $2 \mathrm{C}$ ). The maximum value of the $\mathrm{CH}_{4} / \mathrm{CO}_{2}$ molar ratios in vapor inclusions, determined by the detection limit of Raman spectroscopy, corresponds to a minimum $\log \mathrm{fO}_{2}$ near $\mathrm{NNO}+0.3$, based on the estimation by Ohmoto and Kendrick (1977) that S-type granitoids with a magmatic redox potential near $\mathrm{FMQ} \approx \mathrm{NNO}-1$ yield hydrothermal fluids with a $\mathrm{CH}_{4} / \mathrm{CO}_{2}$ ratio near unity (note that the $\mathrm{CH}_{4}$ to $\mathrm{CO}_{2}$ oxidation reaction involves $2 \mathrm{O}_{2}$ ). In reality, the fluids may have been more reduced (higher $\mathrm{CH}_{4}$ ) if the inclusions had lost $\mathrm{H}_{2}$ by post-entrapment diffusional reequilibration, which would be consistent with chalcopyrite and anhydrite daughter crystals that do not dissolved during heating (Mavrogenes and Bodnar, 1994; Fig. 6). Elevated Fe/Mn ratios in brine inclusions of low-grade Mo-stage samples, compared to lower $\mathrm{Fe} / \mathrm{Mn}$ ratios $\mathrm{Cu}$-stage fluids (Fig. 9) are consistent with an evolution towards more reducing conditions in rock-buffered hydrothermal systems (Boctor, 1985), although it is not possible to quantify this trend for magmatic-hydrothermal conditions.

The proposed explanation for metal separation into distinct $\mathrm{Cu}-\mathrm{Au}$ and Mo precipitation stages requires an evolution in the magmatic fluid source beneath Bingham Canyon. Compared to other porphyry-Cu-Au deposits, the Bingham Canyon system is relatively reduced. This is indicated by the absence of anhydrite from all magmatic rocks and all generations of hydrothermal veins (Klemm et al., 2007; Chambefort et al., 2008) and the lack of hydrothermal magnetite in the barren core of the Bingham system (Arancibia and Clark, 1996; Ulrich and Heinrich, 2001). Molybdenum-rich magmatic sulfides in mafic dikes (Keith et al., 1997) and igneous sulfide minerals in enclaves of fresh monzonite intrusions (Core et al., 2006) are also consistent with relatively reducing magmatic conditions. The reduced nature of the sedimentary country rocks, and the derivation of the Bingham magmas from subcontinental lithospheric mantle that had been metasomatised during the Paleoproterozoic, possibly without direct influence of an oxidized oceanic slab (Pettke et al., 2010), may have both contributed to the relatively reduced nature of all Bingham magmas. Evolution over time of an initially mildly oxidized magma (Cu-Au stage) towards a more reduced magma could be driven by mixing with a new batch of relatively reduced magma or by assimilation of organic-rich sedimentary rocks into the magma chamber below the Bingham Stock. Igneous magnetite crystallization may also contribute to progressive reduction of a magma, by taking ferric iron out of the melts (Sun et al., 2004). Magnetite is an abundant mineral in the EM and probably also in the subjacent batholith, as indicated by a large positive magnetic anomaly (Bankey et al., 1998; Steinberger et al., in prep.).

\section{Evidence of quartz and sulfide redissolution}

The anomalously high but consistent concentrations of $\mathrm{Cu}$ and $\mathrm{S}$ in some fluid inclusion assemblages in the Mo-stage (Fig. 7, 8 and 10) are an expected consequence of local redissolution of copper sulfide initiated in the field of retrograde quartz solubility at 400$500{ }^{\circ} \mathrm{C}$ and 200-500 bar (Fournier, 1999). Since the Mo-stage quartz veins do not host significant copper sulfides, except for some euhedral chalcopyrite in the vein center, varying concentrations of $\mathrm{Cu}$ and $\mathrm{S}$ in the Mo-stage fluids probably do not reflect the direct $\mathrm{Cu}$ sulfide precipitations, but rather indicate $\mathrm{Cu}$ sulfide redissolution.

Cathodoluminescence (CL) imaging shows quartz redissolution followed by precipitation of a second generation of quartz in Mo-stage veins (Fig. 4), similar to textures in the Cu-stage stockwork veins (Redmond et al., 2004; Landtwing et al., 2005; Landtwing et al., 2010). 
Some aliquots of late fluid ascending during in the Mo-stage would have intersected earlier $\mathrm{Cu}$-stage veins, where quartz dissolution would help liberate and redissolve $\mathrm{Cu}$-sulfides into relatively reduced, acidic and perhaps locally higher-temperature Mo-stage fluids. Such redissolution may explain the anomalous enrichment of $\mathrm{Cu}$ and $\mathrm{S}$ in a few fluid inclusion assemblages trapped as secondary trails in the quartz of the Mo stage (Fig. 10). Cu-sulfide redissolution or redistribution has previously been proposed for the Butte (Brimhall, 1979) and El Teniente deposits (Klemm et al., 2007).

\section{Post-entrapment modifications of quartz-hosted fluid inclusion by Cu diffusion}

Experimental studies have shown that the amount of copper in quartz-hosted melt and fluid inclusions can be significantly modified by reequilibraton with surrounding hydrothermal fluids, by selective diffusional exchange of $\mathrm{H}^{+}, \mathrm{Li}^{+}, \mathrm{Na}^{+}, \mathrm{Cu}^{+}$and $\mathrm{Ag}^{+}$through the quartz host (Li et al., 2009; Zajacz et al., 2009). Most recently, it was proposed that the initially trapped high S contents of magmatic-hydrothermal fluid inclusion might enhance their capability to later take up $\mathrm{Cu}$ from external hydrothermal fluids, by inward diffusion and internal precipitation of a $\mathrm{Cu}$-sulfide crystal, to the extent that the activities of $\mathrm{Cu}^{+}, \mathrm{H}^{+}$, and $\mathrm{H}_{2} \mathrm{~S}$ are adjusted to a new chemical equilibrium at lower-temperatures (Lerchbaumer and Audétat, 2011). This post-entrapment process might influence the positive correlations of sulfur and copper concentrations, and the accentuation of 1:2 molar ratios of copper and sulfur in natural quartz-hosted fluid inclusions from many magmatic-hydrothermal ore deposits (Seo et al., 2009). This discovery casts some doubt on the quantification of the proportion of $\mathrm{Cu}$ deposited by vapor or brine during the $\mathrm{Cu}-\mathrm{Au}$ stage of mineralization at Bingham Canyon, but it does not change the conclusions that vapor was the dominant fluid phase throughout the mineralization history (Landtwing et al., 2011; this study). Overestimation of $\mathrm{Cu}-$ concentrations due to post-entrapment diffusional exchange of small singly-charged cations will affect the metal competition for sulfur during selective $\mathrm{Cu}, \mathrm{Au}$ and Mo precipitation, once $\mathrm{Cu}$-Fe-sulfide solubility can be calculated based on future experimentalthermodynamic data. However, it would not negate our proposed mechanism for the separation of $\mathrm{Cu}$-Fe-sulfide and molybdenite precipitation by changing redox and $\mathrm{pH}$ conditions, which is primarily based on analyzed $\mathrm{S}$ and Mo concentrations and reaction stoichiometry.

\section{Concluding Remarks}

Despite the clear separation of molybdenum mineralization from copper - gold mineralization in space and time, the fluids depositing the two distinct ore stages at Bingham Canyon are compositionally similar and follow a similar evolution path. Singlephase magmatic input fluids cooled and expanded into predominant vapor and a minor proportion of condensing brine, and the bulk of the metals were precipitated from the expanding vapor phase. Unless we have missed a distinct precursor fluid, the Mo-stage fluids were not significantly enriched in molybdenum and sulfur. We conclude that oxidized and relatively neutral fluid condition favor precipitation of as chalcopyrite or bornite in the early mineralization stage and initially suppressed molybdenite precipitation. Later-stage fluids may have been more reduced and acid, as a result of a still uncertain evolution of the magmatic fluid source, which could tip the balance and favor molybdenite to become the first-saturating sulfide phase.

Experimental thermodynamic data will be required to evaluate the proposed mechanism of $\mathrm{Cu}$ and Mo separation, but subtle differences in $\mathrm{pH}$ and redox balance could explain the temporal and spatial separation of $\mathrm{Mo}$ and $\mathrm{Cu} \pm \mathrm{Au}$ observed in many other porphyry ore systems, where the two metals commonly occur in distinct vein sets, but in variable proportion, time sequence and deposit-scale spatial zonation. 


\section{Acknowledgments}

This manuscript benefited from constructive reviews by David Cooke, Jacob Hanley and Kirsten Rempel. We thank Larry Meinert for editorial handling. Support by geologists of Kennecott Utah Company, notably Kim Schroeder, during our fieldwork at the Bingham Canyon deposit is gratefully acknowledged. Constructive comments from John Dilles, Kim Schroeder and Geoff Ballantyne on earlier versions of this manuscript are greatly appreciated, and we thank Thomas Wagner, Markus Wälle, Hiroyasu Murakami and Andreas Audétat for discussions about analytical work and geochemical interpretation. We also thank Marion Louvel and Glenn Lambrecht for assistance with Raman analyses at ETH Zurich and University of Bern. This study is funded by Swiss NSF Grants 200020-124906 and 200020-116693/1.

\section{REFERENCES}

Arancibia, O.N., and Clark, A.H., 1996, Early magnetite-amphibole-plagioclase alteration-mineralization in the Island Copper porphyry copper-gold-molybdenum deposit, British Columbia: ECONOMIC GEOLOGY, v. 91, p. 402-438.

Arnorsson, S., and Oskarsson, N., 2007, Molybdenum and tungsten in volcanic rocks and in surface and $<100{ }^{\circ} \mathrm{C}$ ground waters in Iceland: Geochimica et Cosmochimica Acta, v. 71, p. 284-304.

Audétat, A., and Pettke, T., 2006, Evolution of a porphyry-Cu mineralized magma system at Santa Rita, New Mexico (USA): Journal of Petrology, v. 47, p. 2021-2046.

Audétat, A., 2010, Source and evolution of molybdenum in the porphyry $\mathrm{Mo}(-\mathrm{Nb})$ deposit at Cave Peak, Texas Journal of Petrology, v. 51, p. 1739-1760.

Audétat, A., and Pettke, T., 2003, The magmatic-hydrothermal evolution of two barren granites: A melt and fluid inclusion study of the Rito del Medio and Canada Pinabete plutons in northern New Mexico (USA): Geochimica et Cosmochimica Acta, v. 67, p. 97-121.

Bankey, V., Grauch, T., and Kucks, R.P., 1998, Utah aeromagnetic and gravity maps and data, US Geological Survey Open-File Report, 98-761, US Geological Survey.

Beane, R.E., and Titley, S.R., 1981, Porphyry copper deposit; part II. Hydrothermal alteration and mineralization: ECONOMIC GEOLOGY 75 th ANNIVERSARY VOLUME, p. 235269.

Bingen, B., and Stein, H., 2003, Molybdenite Re-Os dating of biotite dehydration melting in the Rogaland high-temperature granulites, $\mathrm{S}$ Norway: Earth and Planetary Science Letters, v. 208, p. 181-195.

Boctor, N.Z., 1985, Rhodonite solubility and thermodynamic properties of aqueous $\mathrm{MnCl}_{2}$ in the system $\mathrm{MnO}-\mathrm{SiO}_{2}-\mathrm{HCl}-\mathrm{H}_{2} \mathrm{O}$ : Geochimica et Cosmochimica Acta, v. 49, p. 565-575.

Bodnar, R.J., and Vityk, M.O., 1994, Interpretation of Microthermometric data for $\mathrm{H}_{2} \mathrm{O}$ $\mathrm{NaCl}$ fluid inclusions: Blacksburg, VirginiaTech, 117-130 p.

Brimhall, G.H., 1979, Lithologic determination of mass-transfer mechanisms of multiple-stage porphyry copper mineralization at Butte, Montana - vein formation by hypogene leaching and enrichment of potassium-silicate protore: ECONOMICGEOLOGY,v. 74, p. 556-589. 158.

Burke, E.A.J., 2001, Raman microspectrometry of fluid inclusions: Lithos, v. 55, p. 139-

Candela, P.A., and Holland, H.D., 1984, The partitioning of copper and molybdenum between silicate melts and aqueous fluids: Geochimica et Cosmochimica Acta, v. 48, p. 373380. 
Cao, X., 1989, Soulubility of molybdenite and the transport of molybdenum in hydrithermal solutions, PhD thesis, Iowa State University, USA.

Chambefort, I., Dilles, J.H., and Kent, A.J.R., 2008, Anhydrite-bearing andesite and dacite as a source for sulfur in magmatic-hydrothermal mineral deposits: Geology,v. 36, p. 719-722.

Chesley, J.T., and Ruiz, J., 1997, Preliminary Re-Os dating on molybdenite mineralization from the Bungham Canyon porphyry copper deposit, Utah, in John, D.A., and Ballantyne, G.H., eds., Geology and Ore Deposits of the Oquirrh and Wasatch Mountains, Utah, 29. Guidebook Series of the Society of Economic Geologists, p. 165-169.

Core, D.P., Kesler, S.E., and Essene, E.J., 2006, Unusually Cu-rich magmas associated with giant porphyry copper deposits: Evidence from Bingham, Utah: Geology, v. 34, p. 41-44.

Cox, D.P., and Singer, D.A., 1988, Distribution of gold in porphyry copper deposits: U.S. Geological Survey Open File Report, p. 88-46.

Crerar, D.A., and Barnes, H.L., 1976, Ore solution chemistry 5. Solubilities of chalcopyrite and chlcocite assemblages in hydrothermal solution at $200 \circ \mathrm{C}$ to $350 \circ \mathrm{C}$ : ECONOMIC GEOLOGY, v. 71, p. 772-794.

Driesner, T., and Heinrich, C.A., 2007, The system $\mathrm{H}_{2} \mathrm{O}-\mathrm{NaCl}$. Part I: Correlation formulae for phase relations in temperature-pressure-composition space from 0 to $1000{ }^{\circ} \mathrm{C}$, 0 to 5000 bar, and 0 to $1 \mathrm{X}-\mathrm{NaCl}$ : Geochimica et Cosmochimica Acta, v. 71, p. 4880-4901.

Einaudi, M.T., Hedenquist, J.W., and Inan, E.E., 2003, Sulfidation state of fluids in active and extinct hydrothermal system: Transition from porphyry to ephithermal environments: Society of Economic Geologist Special Publication 10, p. 285-313.

Etschmann, B.E., Liu, W., Testemale, D., Müller, H., Rae, N.A., Proux, O., Hazemann, J.L., and Brugger, J., 2010, An in situ XAS study of copper(I) transport as hydrosulfide complexes in hydrothermal solutions $\left(25-592^{\circ} \mathrm{C}, 180-600 \mathrm{bar}\right)$ : Speciation and solubility in vapor and liquid phases: Geochimica et Cosmochimica Acta, v. 74, p. 4723-4739.

Fournier, R.O., 1999, Hydrothermal processes related to movement of fluid from plastic into brittle rock in the magmatic-epithermal environment: ECONOMIC GEOLOGY, v. 94, p. 1193-1211.

Günther, D., Audétat, A., Frischknecht, R., and Heinrich, C.A., 1998, Quantitative analysis of major, minor and trace elements in fluid inclusions using laser ablation inductively coupled plasma mass spectrometry: Journal of Analytical Atomic Spectrometry, v. 13, p. 263-270.

Gruen, G., Heinrich, C.A., and Schroeder, K., 2010, The Bingham Canyon Porphyry CuMo-Au Deposit. II. Vein geometry and ore shell formation by pressure-driven rock extension: ECONOMIC GEOLOGY, v. 105, p. 69-90.

Gu, Y., 1993, Theoretical and experimental studies of the hydrothermal geochemistry of molybdenum, PhD thesis, Monash University, Australia.

Guillong, M., and Heinrich, C.A., 2007, Sensitivity enhancement in laser ablation ICPMS using small amounts of hydrogen in the carrier gas: Journal of Analytical Atomic Spectrometry, v. 22, p. 1488-1494.

Guillong, M., Latkoczy, C., Seo, J.H., Günther, D., and Heinrich, C.A., 2008a, Determination of sulfur in fluid inclusions by laser ablation ICP-MS: Journal of Analytical Atomic Spectrometry, v. 23, p. 1581-1589.

Guillong, M., Meier, D.L., Allan, M.M., Heinrich, C.A., and Yardley, B.W.D., 2008b, SILLS: A MATLAB-based program for the reduction of laser ablation ICP-MS data of homogeneous materials and inclusions: Mineralogical Association of Canada Short Course 40, p. 328-333.

Gustafson, L.B., and Hunt, J.P., 1975, Porphyry copper deposit at El-Salvador, Chile: ECONOMIC GEOLOGY, v. 70, p. 857. 
Halter, W.E., Pettke, T., and Heinrich, C.A., 2002, The origin of $\mathrm{Cu} / \mathrm{Au}$ ratios in porphyry-type ore deposits: Science, v. 296, p. 1844-1846.

Heinrich, C.A., 2006, From fluid inclusion microanalysis to large-scale hydrothermal mass transfer in the Earth's interior: Journal of Mineralogical and Petrological Sciences, v. 101, p. 110-117.

Heinrich, C.A., Driesner, T., Stefansson, A., and Seward, T.M., 2004, Magmatic vapor contraction and the transport of gold from the porphyry environment to epithermal ore deposits: Geology, v. 32, p. 761-764.

Heinrich, C.A., Pettke, T., Halter, W.E., Aigner-Torres, M., Audétat, A., Günther, D., Hattendorf, B., Bleiner, D., Guillong, M., and Horn, I., 2003, Quantitative multi-element analysis of minerals, fluid and melt inclusions by laser-ablation inductively-coupled-plasma mass-spectrometry: Geochimica et Cosmochimica Acta, v. 67, p. 3473-3497.

Keith, J.D., Whitney, J.A., Hattori, K., Ballantyne, G.H., Christiansen, E.H., Barr, D.L., Cannan, T.M., and Hook, C.J., 1997, The role of magmatic sulfides and mafic alkaline magmas in the Bingham and Tintic mining districts, Utah: Journal of Petrology, v. 38, p. 1679-1690.

Keppler, H., and Wyllie, P.J., 1991, Partitioning of $\mathrm{Cu}, \mathrm{Sn}, \mathrm{Mo}, \mathrm{W}, \mathrm{U}$, and Th between melt and aqueous fluid in the systems halplogranite- $\mathrm{H}_{2} \mathrm{O} \mathrm{HCl}$ and halplogranite- $\mathrm{H}_{2} \mathrm{O} \mathrm{HF}$ Contributions to Mineralogy and Petrology, v. 109, p. 139-150.

Kesler, S.E., Chryssoulis, S.L., and Simon, G., 2002, Gold in porphyry copper deposits: its abundance and fate: Ore Geology Reviews, v. 21, p. 103-124.

Klemm, L.M., Pettke, T., and Heinrich, C.A., 2008, Fluid and source magma evolution of the Questa porphyry Mo deposit, New Mexico, USA: Mineralium Deposita, v. 43, p. 533-552.

Klemm, L.M., Pettke, T., Heinrich, C.A., and Campos, E., 2007, Hydrothermal evolution of the El Teniente deposit, Chile: Porphyry $\mathrm{Cu}-\mathrm{Mo}$ ore deposition from low-salinity magmatic fluids: ECONOMIC GEOLOGY, v. 102, p. 1021-1045.

Landtwing, M.R., Furrer, C., Redmond, P.B., Pettke, T., Guillong, M., and Heinrich, C.A., 2010, The Bingham Canyon Porphyry Cu-Mo-Au Deposit. III.Zoned Copper-Gold Ore Deposition by Magmatic Vapor Expansion: Economic Geology, v. 105, p. 91-118.

Landtwing, M.R., Pettke, T., Halter, W.E., Heinrich, C.A., Redmond, P.B., Einaudi, M.T., and Kunze, K., 2005, Copper deposition during quartz dissolution by cooling magmatichydrothermal fluids: The Bingham porphyry: Earth and Planetary Science Letters, v. 235, p. 229-243.

Lerchbaumer, L., and Audétat, A., 2011, Preferential partitioning of copper into the vapor phase: fact or a natural artifact?: EGU General Assembly 201, Vienna, Austria, 2011, p. EGU2011-11906.

Li, Y., Audétat, A., Lerchbaumer, L., and Xiong, X.L., 2009, Rapid Na, Cu exchange between synthetic fluid inclusions and external aqueous solutions: evidence from LA-ICPMS analysis: Geofluids, v. 9, p. 321-329.

Mavrogenes, J.A., and Bodnar, R.J., 1994, Hydrogen movement into and out of fluid inclusions in quartz - ExperImental evidence and geological implications: Geochimica et Cosmochimica Acta, v. 58, p. 141-148.

Minubayeva, Z., and Seward, T.M., 2010, Molybdic acid ionisation under hydrothermal conditions to $300^{\circ} \mathrm{C}$ : Geochimica et Cosmochimica Acta, v. 74, p. 4365-4374.

Mountain, B.W., and Seward, T.M., 1999, The hydrosulphide sulphide complexes of copper(I): Experimental determination of stoichiometry and stability at 22 degrees $\mathrm{C}$ and reassessment of high temperature data: Geochimica et Cosmochimica Acta, v. 63, p. 11-29.

Mountain, B.W., and Seward, T.M., 2003, Hydrosulfide/sulfide complexes of copper(I): Experimental confirmation of the stoichiometry and stability of $\mathrm{Cu}(\mathrm{HS})(2)(-)$ to elevated temperatures: Geochimica et Cosmochimica Acta, v. 67, p. 3005-3014. 
Murakami, H., Seo, J.H., and Heinrich, C.A., 2010, The relation between $\mathrm{Cu} / \mathrm{Au}$ ratio and formation depth of porphyry-style $\mathrm{Cu}-\mathrm{Au}+/-$ Mo deposits: Mineralium Deposita, v. 45, p. 1121.

Ohmoto, H., and Kerrick, D., 1977, Devolatilization equilibria in graphitic systems: American Journal of Science, v. 277, p. 1013-1044.

Pettke, T., Oberli, F., and Heinrich, C.A., 2010, The magma and metal source of giant porphyry-type ore deposits, based on lead isotope microanalysis of individual fluid inclusions: Earth and Planetary Science Letters, v. 296, p. 267.

Pokrovski, G.S., Borisova, A.Y., and Harrichoury, J.C., 2008, The effect of sulfur on vapor-liquid fractionation of metals in hydrothermal systems: Earth and Planetary Science Letters, v. 266, p. 345-362.

Redmond, P.B., and Einaudi, M.T., 2010, The Bingham Canyon Porphyry Cu-Mo-Au Deposit. I. Sequence of Intrusions, Vein Formation, and Sulfide Deposition: ECONOMIC GEOLOGY,v. 105, p. 43-68.

Redmond, P.B., Einaudi, M.T., Inan, E.E., Landtwing, M.R., and Heinrich, C.A., 2004, Copper deposition by fluid cooling in intrusion-centered systems: New insights from the Bingham porphyry ore deposit, Utah: Geology, v. 32, p. 217-220.

Rempel, K.U., Migdisov, A.A., and Williams-Jones, A.E., 2006, The solubility and speciation of molybdenum in water vapour at elevated temperatures and pressures: Implications for ore genesis: Geochimica et Cosmochimica Acta, v. 70, p. 687-696.

Rempel, K.U., Williams-Jones, A.E., and Migdisov, A.A., 2008, The solubility of molybdenum dioxide and trioxide in $\mathrm{HCl}$-bearing water vapour at $350{ }^{\circ} \mathrm{C}$ and pressures up to 160 bars: Geochimica et Cosmochimica Acta, v. 72, p. 3074-3083.

Rempel, K.U., Williams-Jones, A.E., and Migdisov, A.A., 2009, The partitioning of molybdenum(VI) between aqueous liquid and vapour at temperatures up to 370 degrees $\mathrm{C}$ : Geochimica et Cosmochimica Acta, v. 73, p. 3381-3392.

Rusk, B.G., Reed, M.H., and Dilles, J.H., 2008, Fluid inclusion evidence for magmatichydrothermal fluid evolution in the porphyry copper-molybdenum deposit at Butte, Montana: ECONOMIC GEOLOGY,v. 103, p. 307-334.

Seedorff, E., and Einaudi, M.T., 2004a, Henderson porphyry molybdenum system, Colorado: I. Sequence and abundance of hydrothermal mineral assemblages, flow paths of evolving fluids, and evolutionary style: ECONOMIC GEOLOGY,v. 99, p. 3-37.

Seedorff, E., and Einaudi, M.T., 2004b, Henderson porphyry molybdenum system, Colorado: II. Decoupling of introduction and deposition of metals during geochemical evolution of hydrothermal fluids: ECONOMIC GEOLOGY, v. 99, p. 39-72.

Seo, J.H., Guillong, M., Aerts, M., Zajacz, Z., and Heinrich, C.A., 2011, Microanalysis of S, $\mathrm{Cl}$, and $\mathrm{Br}$ in fluid inclusions by LA-ICP-MS: Chemical Geology, v. 284, p. 35-44.

Seo, J.H., Guillong, M., and Heinrich, C.A., 2009, The role of sulfur in the formation of magmatic-hydrothermal copper-gold deposits: Earth and Planetary Science Letters, v. 282, p. 323-328.

Sillitoe, R.H., 1997, Characteristics and controls of the largest porphyry copper-gold and epithermal gold deposits in the circum-Pacific region: Australian Journal of Earth Sciences, v. 44, p. 373-388.

Sillitoe, R.H., 2010, Porphyry Copper Systems: ECONOMIC GEOLOGY, v. 105, p. 3-41.

Simon, A.C., Pettke, T., Candela, P.A., Piccolli, P.M., and Heinrich, C.A., 2006, Copper partitioning in a melt-vapor-brine-magnetite-pyrrhotite assemblage: Geochimica et Cosmochimica Acta, v. 70, p. 5583-5600.

Simon, G., Kesler, S.E., Essene, E.J., and Chryssoulis, S.L., 2000, Gold in porphyry copper deposits: Experimental determination of the distribution of gold in the $\mathrm{Cu}-\mathrm{Fe}-\mathrm{S}$ system at $400{ }^{\circ} \mathrm{C}$ to $700{ }^{\circ} \mathrm{C}$ : ECONOMIC GEOLOGY, v. 95, p. 259-270. 
Singer, D.A., Berger, V.I., and Moring, B.C., 2005, Porphyry copper deposits of the world: database, map, and grade and tonnage models., US Geological Survey Open-File Report, US Geological Survey, p. 1060.

Steinberger, I., Hinks, D., Driesner, T., and Heinrich, C.A., in prep., The Bingham Canyon Porphyry Cu-Mo-Au) Deposit. V. Dimensions of a subjacent source intrusion based on geomagnetic and geochemical constraints.: ECONOMIC GEOLOGY.

Sun, W.D., Arculus, R.J., Kamenetsky, V.S., and Binns, R.A., 2004, Release of goldbearing fluids in convergent margin magmas prompted by magnetite crystallization: Nature, v. 431, p. 975-978.

Ulrich, T., Günther, D., and Heinrich, C.A., 1999, Gold concentrations of magmatic brines and the metal budget of porphyry copper deposits: Nature, v. 399, p. 676-679.

Ulrich, T., and Heinrich, C.A., 2001, Geology and alteration geochemistry of the porphyry $\mathrm{Cu}-\mathrm{Au}$ deposit at Bajo de la Alumbrera, Argentina: ECONOMIC GEOLOGY,v. 96, p. 1719-1742.

Ulrich, T., and Mavrogenes, J., 2008, An experimental study of the solubility of molybdenum in $\mathrm{H}_{2} \mathrm{O}$ and $\mathrm{KCl}-\mathrm{H}_{2} \mathrm{O}$ solutions from $500{ }^{\circ} \mathrm{C}$ to $800{ }^{\circ} \mathrm{C}$, and 150 to $300 \mathrm{MPa}$ : Geochimica et Cosmochimica Acta, v. 72, p. 2316-2330.

von Quadt, A., Erni, M., Martinek, K., Moll, M., Peytcheva, I., and Heinrich, C.A., 2011, Zircon crystallization and the life times of ore-forming magmatic-hydrothermal systems: Geology, v. 39, p. 731-734.

Wallace, S.R., 1995, Presidental address; The Climax-type molybdenite deposits What they are, Where they are, and Why they are.: Economic Geology and the Bulletin of the Society of Economic Geologists, v. 90, p. 1359-1380.

White, W.H., Bookstrom, A.A., Kamilli, R.J., Ganster, M.W., Smith, R.P., Ranta, D.E., and Steininger, R.C., 1981, Character and origin of Climax-type molybdenum deposits: ECONOMIC GEOLOGY, v. 75th Anniversary Volume, p. 270-316.

Williams-Jones, A.E., and Heinrich, C.A., 2005, Vapor transport of metals and the formation of magmatic-hydrothermal ore deposits: ECONOMIC GEOLOGY, v. 100, p. 12871312.

Wood, S.A., Crerar, D.A., and Borcsik, M.P., 1987, Solubility of the assemblage pyritepyrrhotite-magnetite-sphalerite-galena-gold-stibnite-bismuthinite-argentite-molybdenite in $\mathrm{H}_{2} \mathrm{O}-\mathrm{NaCl}-\mathrm{CO}_{2}$ solutions from $200^{\circ} \mathrm{C}$ to $350{ }^{\circ} \mathrm{C}$ : ECONOMIC GEOLOGY, v. 82, p. 1864-1887.

Zajacz, Z., and Halter, W., 2009, Copper transport by high temperature, sulfur-rich magmatic vapor: Evidence from silicate melt and vapor inclusions in a basaltic andesite from the Villarrica volcano (Chile): Earth and Planetary Science Letters, v. 282, p. 115-121.

Zajacz, Z., Hanley, J.J., Heinrich, C.A., Halter, W.E., and Guillong, M., 2009, Diffusive reequilibration of quartz-hosted silicate melt and fluid inclusions: Are all metal concentrations unmodified?: Geochimica et Cosmochimica Acta, v. 73, p. 3013-3027.

Zajacz, Z., Seo, J.H., Candela, P.A., Piccoli, P.M., Heinrich, C.A., and Guillong, M., 2010, Alkali metals control the release of gold from volatile-rich magmas: Earth and Planetary Science Letters, v. 297, p. 50-56. 


\section{Table Captions}

Table 1. Descriptions of the quartz vein samples from drill core and mine outcrops that were used for fluid inclusion analyses.

$\mathrm{EM}=$ equigranular monzonite, $\mathrm{QMP}=$ quartz monzonite porphyry, $\mathrm{QLP}=$ quartz latite porphyry.

Table 2. Composition of $\mathrm{Cu}$-stage (quartz stockwork veins) brine inclusion assemblages in the Bingham Canyon deposit.

Averages (Av.; $\mu \mathrm{g} / \mathrm{g}$ ) and standard deviations (SD; 1 sigma) of elements in $\mathrm{Cu}$-stage (quartz stockwork veins). Salinities (Sal.; equivalent $\mathrm{NaCl}$ wt \%) and homogenization temperatures $\left(\mathrm{T}_{h}\right)$ of the assemblages were obtained from microthermometry (Bodnar and Vityk, 1994) and pressures of entrapment were estimated (Driesner and Heinrich, 2007). "Deep stw.", “Cu-vein”, and “QLP stw” represent low Cu grade quartz stockwork veins, high Cu grade quartz stockwork vein, and late-stage (post-QLP) quartz stockwork vein, respectively. Boiling assemblages "boil" allow direct calculation "real PT" of pressures, whereas brine-only "no-boil" assemblages provide minimum estimation "min PT" of pressures. "ND" represents values not determined.

Table 3. Composition of Mo-stage (quartz-molybdenite veins) brine inclusion assemblages

"deep moly", and "Mo-vein" represent low grade quartz-molybdenite veins, and high grade quartz-molybdenite veins, respectively.

Table 4. Concentration of additional elements $(\mathrm{Mn}, \mathrm{Rb}, \mathrm{Sr}, \mathrm{Ba})$ in brine inclusion assemblages in low-grade veins from barren core $\mathrm{Cu}$-stage and Mo-stage in Bingham Canyon deposit.

"Deep stw." and "Deep Moly" represent quartz stockwork veins with low Cu grade and low grade quartzmolybdenite veins, respectively. For the element ratio determinations, we assumed 3,000 $\mu \mathrm{g} / \mathrm{g}$ of $\mathrm{Pb}$ as an internal standard and used analytically-robust Fe/Mn ppm ratios.

Table 5. Compostion of intermediate-density (ID), vapor, and late aqueous fluid inclusion assemblages.

Due to difficulties to get reliable salinities from such low-density fluid inclusions, most of the intermediatedensity (ID) and vapor phase salinities were assumed as 8 equiv. $\mathrm{NaCl}$ wt \% based on previous estimations (Redmond et al., 2004; Landtwing et al., 2010). Only element ratios are analytically-robust and are therefore reported in the current text. Temperatures, pressure data for some vapor inclusions were obtained from brine inclusion data in boiling assemblages.

Table 6. Mass-balance calculation to estimate vapor composition and vapor-brine mass fractions.

Modeling procedure and assumptions following Landtwing et al. (2010, Table 2). Concentrations of intermediate-density (ID) fluid inclusions in deep low Mo grade quartz-molybdenite vein (D50-2155) and concentrations of brine inclusions in boiling assemblage (BT2) in the shallow high Mo grade quartz-molybdenite vein (JH2) were measured by LA-ICP-MS. Input fluid is based on average element ratios of all intermediatedensity fluid inclusions (column c). Element ratios and salinities of brine inclusions in boiling assemblage (column e) were adopted, whereas the poorly known salinities of intermediate-density fluids, vapor and the mass fraction brine and vapor were varied to predict element ratios and salinity of vapor inclusions. The predicted element ratios were compared with analyzed element ratios (column l) to obtain an approximation satisfying all analytical constraints within their estimated uncertainty (Fig. 12). mMCl, mM, and gMCl molar mass of salt components, molar mass of elements, and weight (g) of salt components, respectively. Comp., pred, Conc., 
El-Ratio, and TDS represent composition, predicted value, concentration, element ratio, and total dissolved solids, respectively. 
Figure Captions

Figure 1.

Figure 2.

Figure 3.

Figure 4 .

Figure 5.

Figure 6.

Figure 7.

Figure 8.

Figure 9.

Figure 10.

Figure 11.

Figure 12.

Figure 13. 